University of Louisville

ThinkIR: The University of Louisville's Institutional Repository

Electronic Theses and Dissertations

8-2017

\title{
The DLK1-MEG3 locus in malignant cells of proposed primordial germ cell origins.
}

Zachariah Payne Sellers

University of Louisville

Follow this and additional works at: https://ir.library.louisville.edu/etd

Part of the Cancer Biology Commons, Cell Biology Commons, Developmental Biology Commons, Genetics Commons, and the Molecular Biology Commons

\section{Recommended Citation}

Sellers, Zachariah Payne, "The DLK1-MEG3 locus in malignant cells of proposed primordial germ cell origins." (2017). Electronic Theses and Dissertations. Paper 2747.

https://doi.org/10.18297/etd/2747

This Doctoral Dissertation is brought to you for free and open access by ThinkIR: The University of Louisville's Institutional Repository. It has been accepted for inclusion in Electronic Theses and Dissertations by an authorized administrator of ThinkIR: The University of Louisville's Institutional Repository. This title appears here courtesy of the author, who has retained all other copyrights. For more information, please contact thinkir@louisville.edu. 
THE DLK1-MEG3 LOCUS IN MALIGNANT CELLS OF PROSPECTIVE PRIMORDIAL GERM CELL ORIGINS

\author{
By \\ Zachariah Payne Sellers \\ B.S., University of Kentucky, 2011 \\ M.S., University of Louisville, 2016

\begin{abstract}
A Dissertation
Submitted to the Faculty of the

in Partial Fulfillment of the Requirements

for the Degree of

Doctor of Philosophy

in Microbiology and Immunology

Department of Microbiology and Immunology

University of Louisville

Louisville, KY
\end{abstract} \\ School of Medicine at the University of Louisville
}

August 2017 
Copyright 2017 by Zachariah Payne Sellers

All rights reserved 

THE DLK1-MEG3 LOCUS IN MALIGNANT CELLS OF PROSPECTIVE PRIMORDIAL GERM CELL ORIGINS

\author{
By \\ Zachariah Payne Sellers \\ B.S., University of Kentucky, 2011 \\ M.S., University of Louisville, 2016
}

A Dissertation Approved on

August 8, 2017

By the following Dissertation Committee:

\begin{tabular}{l} 
Dr. Mariusz Ratajczak \\
\hline
\end{tabular}

Dr. Michele Kosiewicz

Dr. Sham Kakar

Dr. Huang-Ge Zhang

Dr. Bing Li 


\section{DEDICATION}

For my family. My rock. 


\section{ACKNOWLEDGMENTS}

First and foremost, I would like to thank Dr. Mariusz Ratajczak for accepting me into his lab as a graduate student before I was ever admitted to a $\mathrm{PhD}$ program. Dr. Ratajczak maintained this faith in me throughout my time in his lab, and he allowed me a level of independence in my work and writing that is not common in this field. I appreciate all the time, effort, and patience he has dedicated to mentoring me throughout the course of my classwork and research. Dr. Ratajczak's enthusiasm for his work is infectious, and I hope to one day relate science to the public the way he can. I would also like to thank Gabriela Schneider for all her help and guidance during this process. I can truly say that Gabriela has seen me at my best and at my worst. I will forever be grateful for the level of patience and selflessness Gabriela displayed throughout the time we worked together. Her guidance is invaluable and I am beyond fortunate for having worked with her. I would like to thank Magda Kucia for our open discussions about VSELs and for all the light she brings to the lab. It has been an honor and a privilege to have casual discussions with the "Mother of VSELs". Magda's humbleness and integrity go unmatched and are true targets for aspiration by any scientist. I would also like to thank Ahmed Abdelbaset Ismail El-Azzazy for his company in the lab and for our great conversations about exercise and the future of stem cells. I would like to thank Malwina Suszyńska, Daniel Pedziwiatr, and Kamila Bujko for all their help and patience with flow cytometry, as well as Janina Ratajczak, Mateusz Adamiak, and all my other fellow lab members for their help during my time in the lab. I 
wish them the best in their scientific careers and especially in their lives outside the lab. I would like to thank Zimple Kurlawala for her support and all the time we've spent laughing at the ridiculous things in life and research. I would like to thank my friends Paul Dascani, Michael Connor, and Nikki Warner for opening their homes to me during my leave of absence and for all the laughs. I would also like to thank my committee members for all their patience and support, and to thank the Department of Microbiology and Immunology at the University of Louisville and its chair Dr. Nejat Egilmez for the support they have provided for me to finish my degree. Finally, I would like to thank Dr. David Allan Butterfield and Dr. Tanea Reed for the support and guidance they've provided to me as a student over the years. I would never have made it into a PhD program if it were not for these great people. 


\begin{abstract}
THE DLK1-MEG3 LOCUS IN MALIGNANT CELLS OF PROSPECTIVE PRIMORDIAL GERM CELL ORIGINS

Zachariah Payne Sellers
\end{abstract}

August 8, 2017

Primordial germ cells (PGCs) are hypothesized to deposit hematopoietic stem cells (HSCs) along their migration route through the embryo during the early stages of embryogenesis. PGCs also undergo global chromatin remodeling, including the erasure and reestablishment of genomic imprints, during this migration. While PGCs do not spontaneously form teratomas, their malignant development into germ cell tumors (GCTs) in vivo is often accompanied by the retention of hypomethylation at the IGF2-H19 imprinting control differentially methylated region (DMR). Previous studies in bimaternal embryos determined that proper genomic imprinting at two paternally imprinted loci was necessary for their growth and development: Igf2-H19 and Dlk1-Meg3. Hypomethylation at DMRs within these two loci confers a tumor-suppressing phenotype, thus provoking the question of whether changes in genomic imprinting at these loci may be important for the development of GCTs. Similarly, these loci were recently implicated in the quiescence and maintenance of HSCs, and there is evidence to suggest that both loci are involved in leukemogenesis. Here, I investigated the DLK1-MEG3 locus in acute myeloid leukemia (AML) patient samples, and discovered significant associations between patient survival and the methylation and expression patterns from this locus. In addition, I investigated the 
methylation of DMRs within the IGF2-H19 and DLK1-MEG3 loci in the human embryonal carcinoma (EC) cell line NTera2 and found that, while the IGF2-H19 control DMR was hypomethylated, the DLK1-MEG3 control DMR and secondary MEG3 DMR were hypermethylated in these cells. The expression ratio of imprinted genes from both loci also agreed with proposed imprinting mechanisms for these phenotypes, and changes in these expression ratios accompanied a decrease in the proliferation rate of these cells during treatment with the DNA methyltransferase inhibitor 5-aza-2'-deoxycytidine. While NTera2 cells functionally responded to exogenous insulin-like growth factors, including IGF2, these cells exhibited strong nuclear staining for DLK1, and shRNA-mediated knockdown of DLK1 revealed a requirement for this gene for the in vitro and in vivo malignant properties of these cells. Furthermore, isolation of potential cancer stem cells (CSCs) from the NTera2 cell line based on CD133 and SSEA4 surface expression produced subpopulations of cells with unique gene expression signatures and migratory characteristics. However, little difference in the DLK1 or OCT4 expression was found among these subpopulations, and the emergence of $\mathrm{CD} 133^{+} \mathrm{SSEA} 4^{+}$cells from in vitroexpanded CD133- ${ }^{-} \mathrm{SSEA}^{-}$, and CD133-SSEA4 ${ }^{-}$singly-sorted cells indicated that, while the overall stemness of these cells was fixed, the phenotype of this established cell line is actually in flux. In conclusion, DLK1 is a potential target to treat AML and EC, meriting future investigations into the development of DLK1-targeting therapies, including the use of specific antibodies, aptamers, and vaccination strategies. EC cell growth and metastasis could also be inhibited by employing DNA methyltransferase inhibitors, and investigations into the effect of these drugs on the expression of genes from the DLK1-MEG3 locus in 
AML could provide valuable information for the development of patient-specific treatments for this disease. 


\section{TABLE OF CONTENTS}

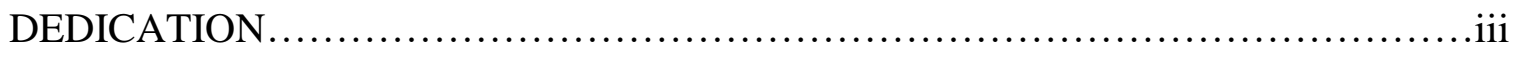

ACKNOWLEDGEMENTS.............................................................

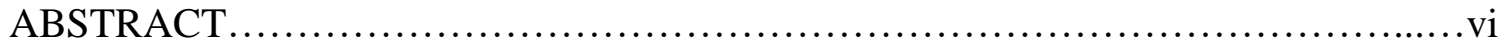

LIST OF TABLES ...............................................................

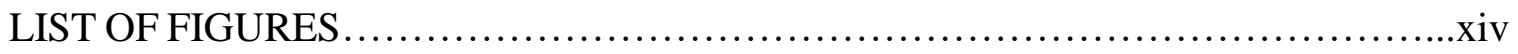

CHAPTER 1: INTRODUCTION .................................................

The maintenance of normal and malignant stem cells by paternally imprinted

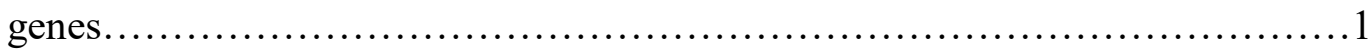

The IGF2-H19 and DLK1-MEG3 loci in embryogenesis and malignancy...........5

Tumor heterogeneity and fluctuating cancer stem cell phenotypes................17

CHAPTER 2: MATERIALS AND METHODS .....................................20

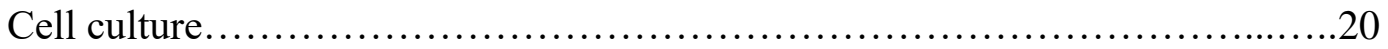

Isolation of human mononuclear cells from umbilical cord blood.................20

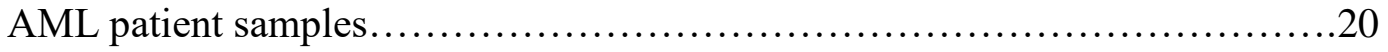

Testicular germ cell tumor tissues.......................................21

Combined bisulfite-restriction analysis and bisulfite sequencing of genomic

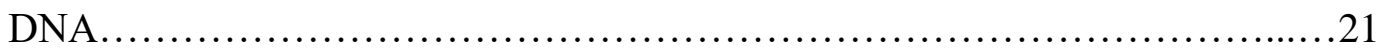

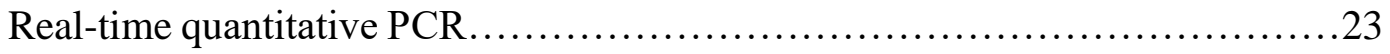


NTera2 treatment with 5-aza-2'-deoxycytidine

Plasmid preparation and transfection of NTera2 cells.......................24

Proliferation assays of transfected NTera 2 cells...........................25

Tumor growth in immunodeficient mice................................. 25

Organ seeding efficiencies........................................... 25

Proliferation assays of NTera2 cells treated with insulin-like growth factors......26

Western blot.....................................................27

Immunofluorescence................................................ 27

Flow cytometry analysis of the IGF1 receptor and insulin receptor.............28

Fluorescence-activated cell soring....................................29

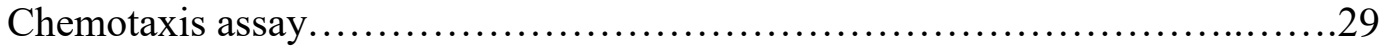

Single-cell expansion and FACS analysis................................ 30

Statistical analysis................................................. 30

CHAPTER 3: DLK1 EXPRESSION AND MEG3 PROMOTOR METHYLATION ARE NOVEL MARKERS OF AML PATIENT SURVIVAL ............................

Introduction......................................................... 36

Results..........................................................43

Methylation at select $\mathrm{CpG}$ sites within the DLK1-MEG3 locus is associated with AML patient outcomes...................................43

DLK1 expression is associated with AML patient outcomes, but not with CpG site methylation.........................................53

Discussion........................................................... 64 
Conclusions

CHAPTER 4: GENOMIC IMPRINTING IDENTIFIES DLK1 AS A NOVEL THERAPEUTIC TARGET FOR EMBRYONAL CARCINOMA $\ldots \ldots \ldots \ldots \ldots \ldots \ldots \ldots . . \ldots 70$

Introduction...................................................... 70

Results........................................................... 72

Hypomethylation at the paternally imprinted $I G F 2-H 19$ locus in NTera2 correlates with a low $I G F 2 / H 19$ expression ratio....................72 Hypermethylation at the paternally imprinted DLK1-MEG3 locus in NTera2 correlates with a high $D L K 1 / M E G 3$ expression ratio.................76 The demethylating compound 5-aza-2'-deoxycytidine inhibits the in vitro growth of NTera2 while demethylating the MEG3 promotor and augmenting the DLK1/MEG3 and IGF2/H19 expression

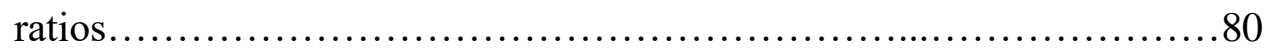

NTera2 expresses insulin-like growth factor-related genes..............91

Insulin-like growth factors phosphorylate the mitogenic signaling molecules AKT and MAP kinase and stimulate NTera2 proliferation in vitro in serum-free conditions.

DLK1 downregulation decreases NTera2 proliferation in vitro and NTera2 tumor size and organ seeding abilities in vivo........................95 Embryonal carcinoma tissue samples exhibit PGC-like imprinting at the IGF2-H19 and DLK1-MEG3 loci. 101 
Cancer stem cell surface markers CD133 and SSEA4 mark distinct subpopulations of NTera2 with unique gene expression signatures of cell stemness.................................................... 103 NTera2 subpopulations sorted by CD133 and SSEA4 surface expression display unique migratory characteristics in vitro and in vivo.............109 Each NTera2 subpopulation sorted by CD133 and SSEA4 surface expression re-establishes the CD133- and SSEA4-expressing phenotypes of the parental NTera 2 cell line upon in vitro expansion........................112

Discussion......................................................... 114

Conclusions.......................................................... 120

CHAPTER 5: CONCLUSIONS AND FUTURE DIRECTIONS........................122

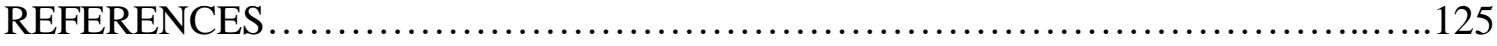

CURRICULUM VITAE........................................................ 134 


\section{LIST OF TABLES}

TABLE

PAGE

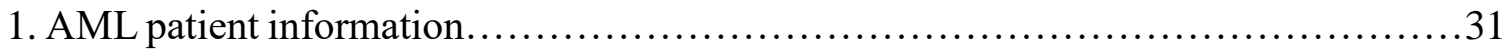

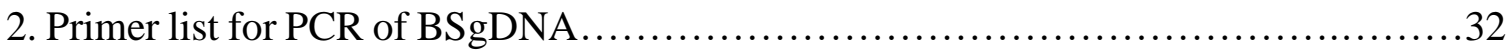

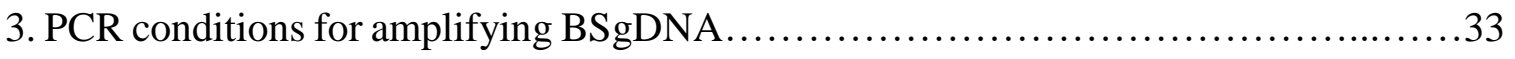

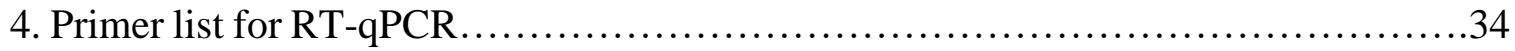

5. Plasmid shRNA sequences for producing stably transfected cell lines................35

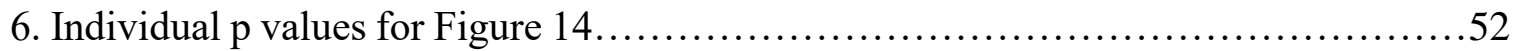

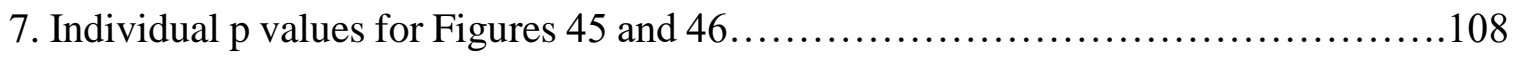

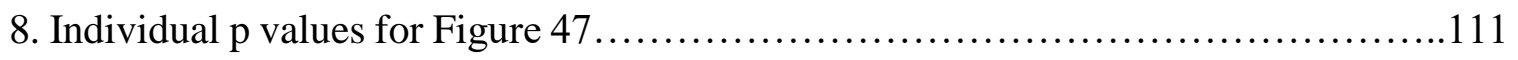




\section{LIST OF FIGURES}

FIGURE

PAGE

1. The $I G F 2-H 19$ locus.......................................................

2. Genomic imprinting at the IGF2-H19 and DLK1-MEG3 loci....................

3. Opposing roles in growth by tandemly expressed genes..........................14

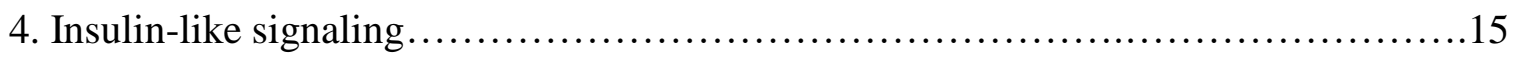

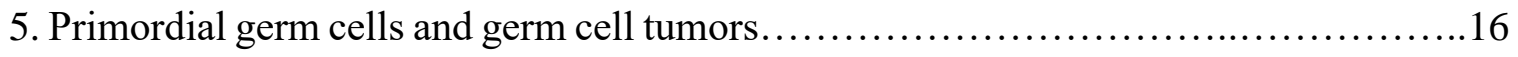

6. Leukemic cells exhibit differences in methylation at imprinted gene network loci compared to mononuclear cells.............................................

7. Methylation at imprinted gene network loci is not associated with complete remission in

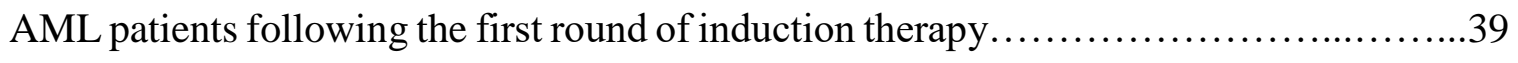

8. Methylation at imprinted gene network loci is not associated with AML patient

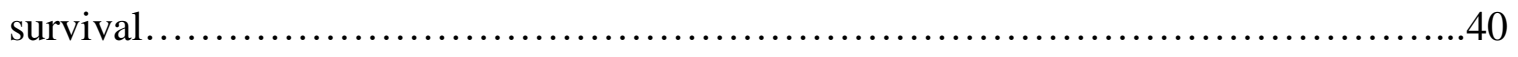

9. CpG sites within the DLK1-MEG3 locus................................42

10. Leukemic cells exhibit differences in methylation at CG6, CG7, and CG8 within the DLK1-MEG3 locus compared to mononuclear cells............................47

11. Methylation at individual $\mathrm{CpG}$ sites within the DLK1-MEG3 locus is not associated with complete remission in AML patients following the first round of induction therapy..48 12. Methylation at CG7 and CG9 within the promotor region of MEG3 is associated with AML patient survival...................................................49 
13. Methylation at CG7 in the promotor region of $M E G 3$ does not exhibit age-related associations with complete remission in AML patients following the first round of

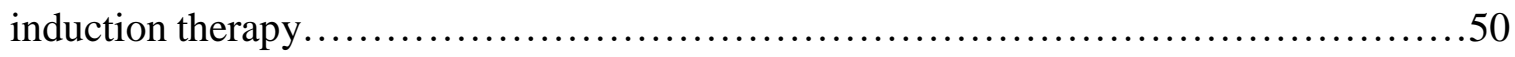

14. Combining methylation results from multiple $D L K 1-M E G 3 \mathrm{CpG}$ sites uncovers unique associations between AML patient survival and methylation at this locus...............51

15. Leukemic cells exhibit lower $D L K 1$ and $M E G 3$ expression than mononuclear cells...55

16. DLK1 and MEG3 expression is not associated with complete remission in AML

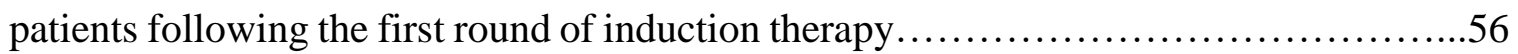

17. $D L K 1$ expression is associated with AML patient survival........................57

18. DLK1 and MEG3 expression is not associated with methylation at CG6............58

19. DLK1 and MEG3 expression is not associated with methylation at CG7 .............59

20. DLK1 and MEG3 expression is not associated with methylation at CG8.............60

21. DLK1 and MEG3 expression is not associated with methylation at CG9............61

22. DLK1 and MEG3 expression is not associated with combined methylation results from

CG6-9.

23. DLK1 and MEG3 expression is not associated with combined methylation results from CpG sites within the $M E G 3$ promotor region.

24. NTera2 cells exhibit hypomethylation at the IGF2-H19 ICR .74 25. A low IGF2/H19 expression ratio in NTera2 cells reflects their erasure of imprinting

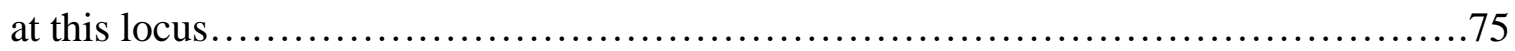
26. NTera2 cells exhibit hypermethylation at DMRs within the DLK1-MEG3 locus......77 27. A high DLK1/MEG3 expression ratio in NTera2 cells reflects their loss of imprinting at this locus... .78 
28. NTera2 cells exhibit a strong nuclear presence of the DLK1 protein

29. 5-azaD treatment of NTera2 cells inhibits their proliferation and demethylates the

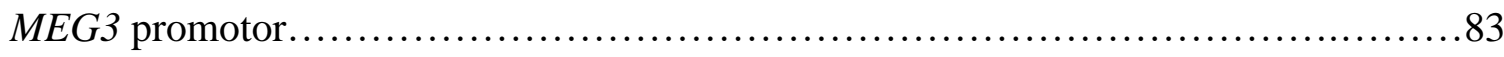

30. 5-azaD treatment of NTera2 cells augments their expression of genes from the DLK1MEG3 locus

31. 5-azaD treatment of NTera2 cells augments their expression of genes from the IGF2H19 locus

32. 5-azaD treatment of NTera2 cells decreases their expression of the pluripotency marker OCT4 .86

33. NTera2 cells exhibit low methylation at maternally imprinted loci

34. NTera2 cells exhibit hypermethylation at the P57-LIT1 locus, which is slightly demethylated by $5-\mathrm{azaD}$. .88

35. A high P57/LIT1 expression ratio in NTera2 cells reflects their loss of imprinting at this locus.

36. 5-azaD treatment of NTera2 cells augments their expression of genes from the P57LIT1 locus. .90

37. NTera 2 cells express receptors for insulin-like signaling growth factors

38. Insulin-like signaling growth factors stimulate NTera2 cells.

39. DLK1 knockdown decreases NTera2 OCT4 expression and in vitro cell

proliferation.

40. DLK1 knockdown cells exhibit strong nuclear DLK1 staining....................98

41. DLK1 knockdown decreases NTera2 tumor growth in vivo 
42. DLK1 knockdown decreases the in vivo organ seeding efficiencies of NTera2

cells. ....

100

43. Human primary EC tissue exhibits hypomethylation at the IGF2-H19, DLK1-MEG3, and P57-LIT1 loci. 102

44. Sorting strategy for NTera2 subpopulations based on CSC markers CD133 and

SSEA4.

45. The CSC markers CD133 and SSEA4 distinguish subpopulations of NTera2 cells exhibiting unique gene expression signatures .106

46. NTera2 subpopulations exhibit differences in their expression of imprinted genes....

47. NTera2 subpopulations demonstrate unique migratory abilities in vitro and in vivo 110

48. Singly sorted NTera2 cells exhibit a fluctuating phenotype upon in vitro expansion. 


\section{CHAPTER 1: INTRODUCTION}

\section{The maintenance of normal and malignant stem cells by parentally imprinted genes}

Of the many layers of control over gene transcription, the developing field of epigenetics has characterized several highly complex, dynamic processes which govern transcription at arguably the most basic level: genomic availability. Broad changes to the chromatin landscape occur through a growing list of post-translational modifications to histone proteins in coordination with environmental conditions such as cellular metabolism [1]. This is exemplified by the transition from quiescence to proliferation in skeletal muscle stem cells, which occurs in response to metabolic changes that promote acetylation at the lysine 16 residue of histone $\mathrm{H} 4$ [2].

In addition to these dynamic processes which allow for chromatin "breathing", epigenetic processes are also at the core of cellular identity. Indeed, this epigenetic tagging system, collectively known as the epigenome, can help distinguish certain cell types [3]. For example, the process of creating induced pluripotent stem cells (iPSCs) can generate cells which are functionally very similar, but permanent changes to the epigenetic landscape as side effects of the induction process dictate the true stem cell potency, or stemness, of these cells [4]. In this case, the differences in stemness between these cells results from epigenetic marks which are localized to one specific region of the genome. This region, known as the $D L K 1-M E G 3$ locus, participates in an epigenetic inheritance

mechanism - genomic imprinting - which is unique to only 100 known genes in the human genome sequestered into even fewer loci (www.geneimprint.com). Genomic imprinting 
controls the dosage of genes within a locus via differential chemical modifications to the maternally and paternally inherited chromosomes. For iPSCs, treatment with the histone deacetylase inhibitor valproic acid (VPA) induced changes to the imprinting pattern at the DLK1-MEG3 locus, as measured by increases in the activating histone modifications $\mathrm{H} 3$ acetylation and $\mathrm{H} 3 \mathrm{~K} 4$ methylation and by decreases in the methylation of differentially methylation regions (DMRs) within this locus. While this illustrates the cooperation of several epigenetic marks to influence the overall imprinting pattern at a locus, DMR methylation is among the most common measures of genomic imprinting. Figure 1 illustrates the archetype mechanism of gene regulation by methylation at DMRs, where allele-specific methylation controls the binding of chromatin-modifying proteins such as CCCTC-binding factor (CTCF) to promote the transcription of certain genes only from either the maternally- or paternally-inherited chromosome.

Coincidentally, the archetype imprinted locus illustrated in Figure 1, IGF2-H19, is also a major player in regulating cellular identity. Different populations of hematopoietic stem cells (HSCs) were recently shown to express growth restriction-associated imprinted genes in accordance with their placement in the HSC hierarchy, where the highest expression of these genes, including H19 and Meg3, was found in the long-term repopulating HSC (LT-HSC) population at the top of this hierarchy [5]. This group also found that disruption of genomic imprinting at the Igf2-H19 locus via deletion of the imprinting control region (ICR), the control DMR for this locus, promoted the loss of quiescence by LT-HSCs and the accumulation of more differentiated HSCs over time. Interestingly, later work uncovered a similar role for the Dlkl-Meg3 locus in maintaining HSCs, where the deletion of either the intergenic control (IG) DMR or the secondary Meg3 
DMR combined with the first five introns of $\mathrm{Meg} 3$ resulted in decreased noncoding RNA (ncRNA) expression from this locus and decreased fetal liver HSC numbers [6, 7]. In addition, quiescent adult pluripotent stem cells which exhibit hypomethylation at the IGF2H19 ICR, named very small embryonic-like stem cells (VSELs), displayed increases in methylation at the ICR upon in vitro expansion and differentiation $[8,9]$. Thus, genomic imprinting at these loci is especially important for maintaining the stemness of somatic and pluripotent stem cells. Furthermore, downregulation of the H19 gene in embryonal carcinoma (EC) cells, which are hypothesized to be the cancer stem-like cells (CSC) that give rise to more differentiated germ cell tumor tissues, was accompanied by decreases in pluripotency gene expression in these cells, and $D L K 1$ has been identified as a CSC marker which is important for the malignancy of CSCs in various cancers such as hepatocellular carcinoma and neuroblastoma [10,11]. Taken together, the IGF2-H19 and DLK1-MEG3 loci not only govern the maintenance of somatic and pluripotent stem cells, but genes from these loci are also involved in CSC malignancy. Considering the unique importance of these paternally imprinted genes in embryonic growth and development, it is not surprising that aberrant imprinting at these loci is also seen in certain malignancies [12, 13]. 


\section{IGF2-H19}
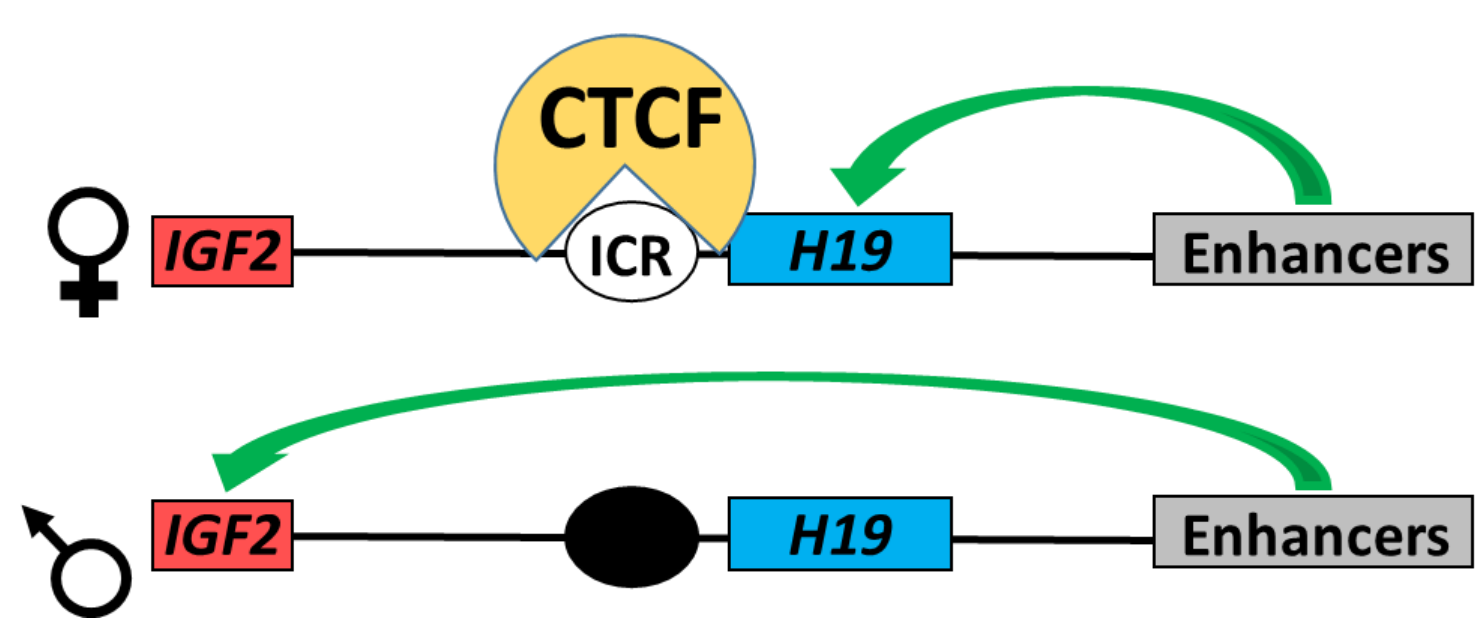

Figure 1. The IGF2-H19 locus

Genomic imprinting at the IGF2-H19 locus involves the binding of a CTCF protein to the ICR to regulate gene expression. In somatic cells, the ICR is hypomethylated on the maternally inherited chromosome (unfilled oval) and hypermethylated on the paternally inherited chromosome (filled oval). This methylation pattern controls the binding of CTCF proteins to the ICR, which allows for downstream enhancers to act on the promotor of either $H 19$ or $I G F 2$. 


\section{The IGF2-H19 and DLK1-MEG3 loci in embryogenesis and malignancy}

The regulation of gene transcription by DMR methylation is well characterized for the IGF2-H19 locus (Figure 1) [14]. Though the exact mechanism of genomic imprinting for this locus is not yet fully described, the importance of the ICR for gene transcription was previously shown in a seminal paper by Kono, et al., in which bimaternal mice were generated for the first time via manipulation of only the Igf2-H19 locus [15]. Gametes carry specific chemical modifications to their chromatin, including DMR methylation and posttranslational modifications to histone proteins, such that the fusion of egg and sperm causes these chemical modifications to complement each other at imprinted loci. This phenomenon allows for unique gene expression ratios from imprinted loci based on the enhanced opportunity for transcription from either the paternally inherited or maternally inherited chromosome (Figure 2). Maternally imprinted loci are those which are chemically modified primarily on the maternally inherited chromosome and represent the majority of known imprinted loci. In mice, the Igf2-H19 and Dlkl-Meg3 loci are two of only four known loci which are paternally imprinted [16]. Thus, the fusion of oocytes does not provide complementing chemical modifications at imprinted loci, preventing the dosage of imprinted gene expression which is found in a normal conceptus and preventing the proper growth and development of the resulting embryo, and work by Kono, et al. highlighted the specific importance of the paternally imprinted $\mathrm{Ig} f 2-\mathrm{H} 19$ locus in overcoming this restriction for creating viable bimaternal mice.

Kono, et al. found that fusing one normal oocyte with one oocyte from which the Igf2-H19 ICR and the $\mathrm{H} 19$ gene were deleted overcame the growth restriction which accompanied oocyte fusion and resulted in the full-term development of mice [15]. 
Importantly, while this process was very inefficient and resulted in the birth of only two surviving pups from 371 implanted embryos, the single pup which was sacrificed displayed a higher Dlk1/Meg3 transcription ratio than the non-surviving pups generated in the same manner. This indicated that the Dlkl-Meg3 locus was also important for bimaternal mouse generation, and later work showed that manipulation of both the Igf2-H19 and Dlkl-Meg3 loci enhanced the efficiency of this process [17].

In light of later work investigating genes from the IGF2-H19 and DLK1-MEG3 loci, these results for bimaternal mice generation may be explained in terms of the maintenance of cell stemness. As previously stated, the expression of ncRNAs from these loci maintains HSC stemness and numbers, and the lncRNA H19 preserves the stemness of EC cells $[5,6,18]$. Similarly, deletion of the $H 19$ gene on the maternal chromosome results in larger teratomas, which are composed of tissues derived from differentiated EC cells [19]. Interestingly, deletion of $\mathrm{Meg} 3$ from the maternal chromosome results in the development of larger teratomas as well [20]. This Meg3 deletion also results in the decreased expression of ncRNAs from the Dlkl-Meg3 locus, including miRNAs which are important for assessing the stemness of iPSCs, as well as the decreased expression stem cell-specific miRNAs. Taken together, it appears that downregulation of ncRNAs from the IGF2-H19 and DLK1-MEG3 locus decreases the stemness of cells and promotes their differentiation. In the case of teratomas, this results in the increased accumulation of bulk differentiated tumor tissue. For bimaternal embryos, a similar situation likely occurs whereby tissue development is impaired due to the high expression of ncRNAs from these loci, as illustrated for the erasure of imprint in Figure 2 which represents the imprint found at these loci as the result of oocyte fusion. Relaxing the expression of these ncRNAs via 
manipulation of both loci allows for the proper differentiation of stem cells into developing embryonic tissues, mimicking the proper somatic imprint which is found in somatic cells and ESCs (Figure 2) [8]. The decreased expression of protein-coding genes Igf2 and DlkI is likely a major player in the growth restriction of bimaternal embryos as well, since Igf2 and Dlk1 are deeply involved in early growth and development.

The parent-offspring conflict theory posits that parents contribute differently towards the growth and development of their offspring [21]. In the context of genomic imprinting at the IGF2-H19 and DLK1-MEG3 loci, this is demonstrated by the expression of growth-promoting protein-coding genes from the paternally inherited allele and growthsuppressing noncoding RNAs (ncRNAs) from the maternally inherited allele in cells with a somatic imprint (Figure 3), with bimaternal embryo generation resulting in low expression of these protein-coding genes. Insulin-like growth factor 2 (IGF2), encoded by the IGF2 gene, participates in insulin-like signaling via activation of the IGF1 receptor and insulin receptor to promote cell growth and proliferation (Figure 4). Delta-like homologue 1 (DLK1), encoded by the $D L K 1$ gene, participates in cell-specific growth-related activities of which the exact mechanisms are largely uncharacterized. While Igf2 is known to stimulate early embryonic growth, studies in mice have found that a balance between adult HSC self-renewal and differentiation is dependent on $I g f 2$ expression, and miRNAs from the Igf2-HI9 and DlkI-Meg3 loci actually decrease the expression of proteins involved in insulin-like signaling and the pro-growth mTOR pathway [5, 6, 22]. A recent study described an $I G F 2$ variant in humans which severely restricted postnatal growth when inherited paternally, similar to the prenatal growth retardation found in mice who have a disrupted Igf2 gene on the paternally inherited chromosome [23, 24]. Dlk1-null mice are 
also growth retarded and have lower survival rates compared to wild-type mice [25, 26]. One proposed mechanism for DLK1 action is through the inhibition of NOTCH signaling, and the overlapping expression patterns of Dlk1 protein and Notch mRNA in embryonic tissues led to the idea that Dlk1 inhibits the Notch-induced differentiation of these tissues [27]. In contrast to Dlk1-null mice, transgenic mice expressing Dlkl at twice the normal level exhibit overgrowth with a failure to thrive in early life, and mice with three times the Dlkl expression of normal mice exhibit organ abnormalities and embryonic lethality [28]. Interestingly, in contrast to the effects of increased Igf2 expression on HSC differentiation, this increased $D l k l$ expression in transgenic animals impaired the maturity of certain tissues, in line with previous observations and the suggestion that a balance in proliferation and differentiation of tissues is skewed by defects in imprinted gene expression from DlklMeg3 [29]. In support of this, the upregulation of Dlkl was found to occur during the prolonged culture of murine ESCs - a situation which has been found to accompany higher proliferation rates of these cells and their development of EC-like malignant characteristics - suggesting that $D l k l$ expression in the embryo might promote the expansion of certain stem cells such as ESCs and prevent their differentiation into embryonic tissues [30, 31]. Furthermore, though no association between DLKl expression and EC has been investigated, aberrant imprinting has been observed in the childhood cancer rhabdomyosarcoma, with hypermethylation of the IG-DMR observed in the embryonal histological subtype [12]. Considering this requirement for the balanced expression of growth-promoting protein-coding genes from the IGF2-H19 and DLK1-MEG3 loci in the proliferation and differentiation of stem cells, it is not surprising that $I G F 2$ and $D L K 1$ are involved in several malignancies, or that DLK1 has been characterized as a potential cancer 
stem cell (CSC) marker which promotes the stemness of cancerous cells [11, 32-35]. As GCTs exhibit hypomethylation of the IGF2-H19 locus, autocrine IGF2 expression is not likely to contribute significantly to the growth of these tumors [36-38]. Investigations into the imprinting of other loci such as DLK1-MEG3 are lacking in GCTs such as EC, and these studies may prove important considering the epigenome dynamics of the primordial germ cells (PGCs) from which these tumors likely originate [36].

As the precursors of gametes, primordial germ cells (PGCs) first appear in the developing epiblast, then undergo extensive chromatin remodeling which erases and reestablishes genomic imprinting patterns [39, 40]. Interestingly, there are significant overlaps between the transitions into and out of proliferation by PGCs and changes to their epigenome (Figure 5A) [41]. Similarly, this timeframe also overlaps with the first signs of hematopoiesis in the embryo [42]. Importantly, though these cells are derivatives of ESCs, which are capable of forming teratomas in vivo, PGCs are not able to form teratomas. However, the fact that hypomethylation of the IGF2-H19 ICR is commonly found in GCTs indicates that these tumors likely originate from the germ cell compartment [36-38]. This would be surprising given that the IGF2-H19 locus is responsible for the quiescence of HSCs and that maternal $H 19$ deletion promotes the growth of teratomas. However, the role of H19 in maintaining the expression of pluripotency markers in EC cells, which may be the stem cell responsible for certain types of GCTs (Figure 5C), suggests that this imprinting pattern may be important for GCT development $[18,43]$.

Like GCTs, populations of quiescent adult stem cells were recently described in mice and humans which also share many characteristics with epiblast stem cells and PGCs [44]. These VSELs have thus far been best characterized in mice, where they exhibit 
hypomethylation of the Oct4 promotor and, like GCTs, also exhibit hypomethylation of the Igf2-H19 ICR [8]. Like PGCs, these cells do not proliferate in vitro, do not form teratomas in vivo, and do not complement blastocyst development. However, VSELs can differentiate into all three germ layers in vitro and enter the cell cycle in vivo in response to various stimuli [45-47]. Collectively, these characteristics arguably place VSELs near the top of the adult stem cell hierarchy. Surprisingly, successful expansion of these cells was very recently reported to occur in response to treatment with VPA, a compound which was also shown to enhance the ex vivo expansion of HSCs while promoting their expression of $D L K 1$, suggesting that an increase in $D L K 1$ expression may also be related to the exit from quiescence by VSELs $[9,48]$. In contrast to their hypomethylated Igf2-H19 locus, VSELs exhibit somatic-like methylation at the Dlk1-Meg3 locus, indicating that this entire locus is "open" for activation by VPA, similar to the activation of this locus by VPA during the generation of iPSCs [4]. In total, these studies put significant focus on the IGF2-H19 and $D L K 1-M E G 3$ loci as potentially important players in the proliferation of quiescent cells which are proposed to arise from PGCs in the developing embryo. Interestingly, multiple groups have reported that VSELs can give rise to hematopoietic cells in vitro, suggesting that VSELs represent an Oct4-expressing "missing link" between PGCs and hematopoiesis in the developing embryo and posing questions regarding the potential roles of VSELs in the development of GCTs and leukemias [47, 49].

A common characteristic which is shared between PGCs and GCTs is the expression of pluripotency markers such as $O C T 4$ and NANOG. EC is a type of GCT which exhibits certain aspects of pluripotency, including the ability to differentiate into all three germ layers [50]. PGCs, while not inherently pluripotent, are able to acquire pluripotency 
upon transformation into embryonic germ cells (EGCs) in vitro, and a developmental fork in the road has been proposed for the controlled and malignant paths to pluripotency by PGCs (Figure 5B) [51]. Importantly, studies in testicular GCTs (TGCTs) have resulted in models which place EC as the precursor cells to more differentiated TGCTs (Figure 5C) [43]. Indeed, EC is considered the malignant counterpart to ESCs and the stem-like cells of teratocarcinomas [30]. Interestingly, the DNA methyltransferase inhibitor 5-aza-2'deoxycytidine (5-azaD) is toxic to EC cells at low doses in vitro, and this compound was recently found to target only the stem-like cells of teratocarcinomas for apoptosis and not more differentiated cells $[52,53]$. While TGCTs are easily treated and most often cured, EC is commonly found within mixed GCTs, and if EC is the primary component of the GCTs then there is a high probability of metastatic spread by the time of diagnosis [54]. 5$\mathrm{azaD}$ is currently used to treat certain myelodysplastic syndromes (MDSs) and boasts a high safety profile, making it an interesting candidate drug for the treatment of EC [55].

Considering that MDSs originate in the HSC compartment, and that HSCs are proposed to originate from migrating PGCs, it is interesting that several reports have documented associations between GCTs and leukemias such as acute myeloid leukemia (AML) [5, 56-58]. Similarly, as the quiescence and stemness maintenance of murine HSCs was determined to be controlled by the Igf2-H19 and Dlkl-Meg3 loci, it is not surprising that the imprinting of these loci is disrupted in AML $[5,6,59,60]$. The DLK1-MEG3 locus in particular has been extensively studied in MDS and leukemias, where MEG3 was identified as a tumor suppressor in AML and $D L K 1$ was found to inhibit the differentiation and proliferation of promyelocytic cells [61, 62]. However, few studies have focused on the potential associations between genes from these paternally imprinted loci and patient 
outcome. In MDS, which may evolve to AML, $D L K 1$ expression was found to correlate with the blast percentage of bone marrow cells in MDS and increased as the disease progressed [63]. Considering the importance of these loci in the maintenance of stem cells, and especially the roles of DLK1 in CSC maintenance, the potential for CSCs to be responsible for the high relapse rate in AML highlights the importance of investigations into the roles of genes from these loci in the clinical response and survival of AML patients [64]. 


\section{$\underline{\text { IGF2-H19 }}$}

Proper Somatic Imprint

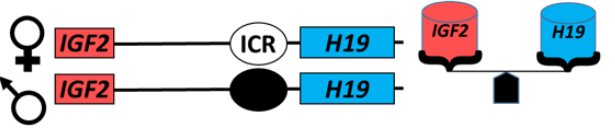

Erasure of Imprint

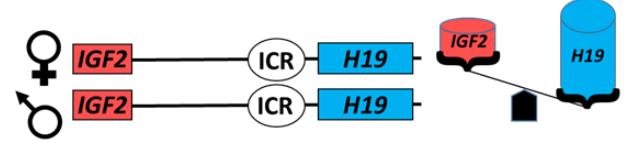

Loss of Imprint

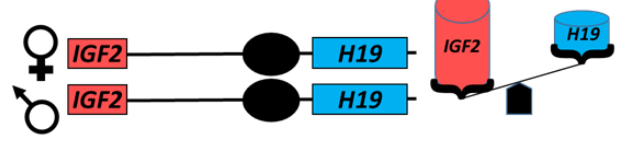

DLK1-MEG3

Proper Somatic Imprint

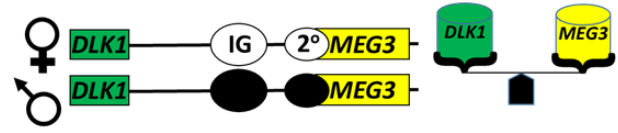

Erasure of Imprint

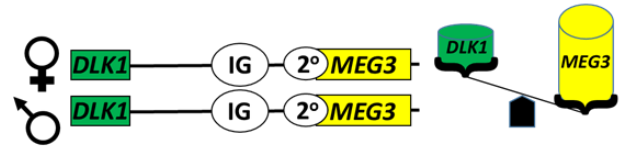

Loss of Imprint

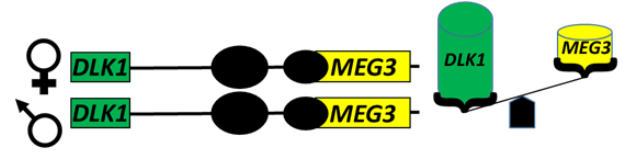

Figure 2. Genomic imprinting at the IGF2-H19 and DLK1-MEG3 loci

Genomic imprinting as a function of methylation at DMRs within the IGF2-H19 and DLK1-MEG3 loci controls the balance of expression for their respective ncRNAs and protein-coding genes. Hypomethylation (unfilled ovals) of the ICR, intergenic control DMR (IG-DMR; IG), and secondary MEG3 DMR $\left(2^{\circ}\right)$ promotes the expression of ncRNAs from these loci, whereas hypermethylation (filled ovals) of these DMRs promotes the expression of protein-coding genes. 


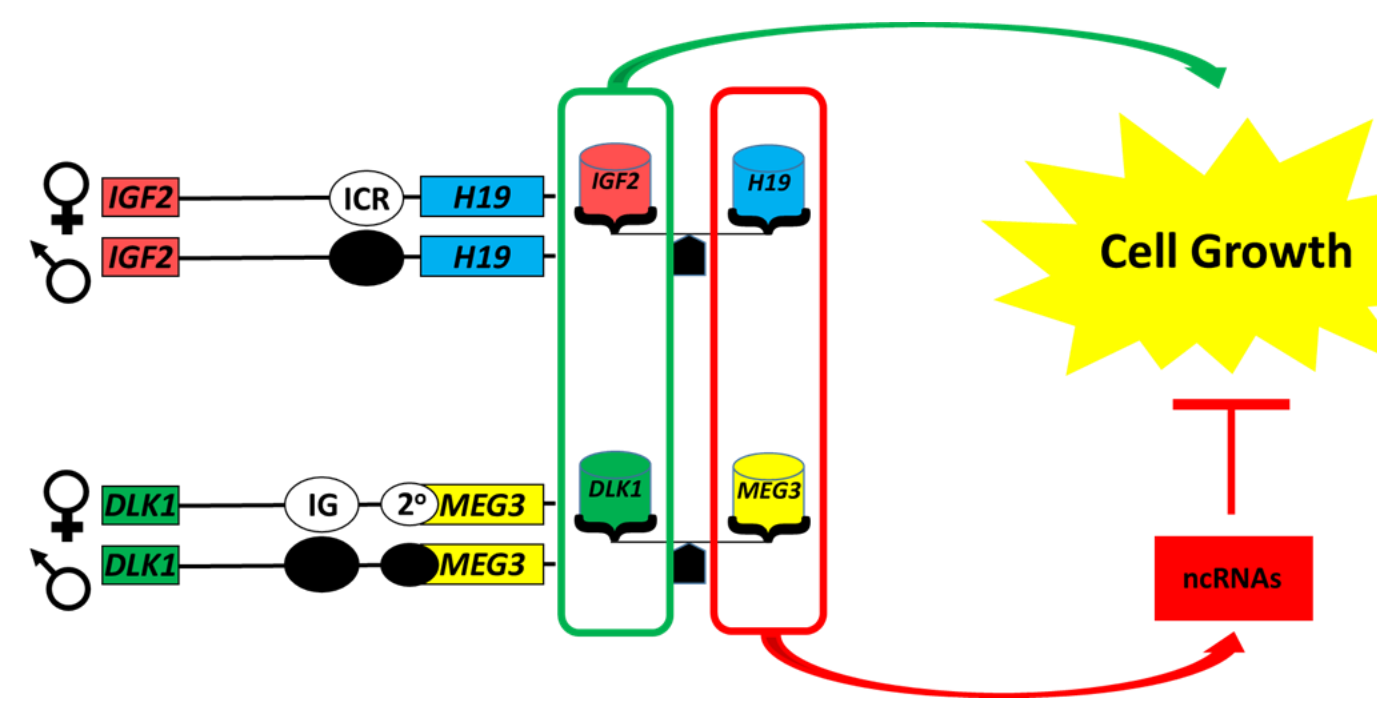

Figure 3. Opposing roles in growth by tandemly-expressed genes

The protein-coding genes and ncRNAs expressed from the IGF2-H19 and DLK1-MEG3 loci exhibit opposing roles in cell growth. Hypomethylation (unfilled ovals) of the ICR, IG-DMR (IG), and secondary MEG3 DMR $\left(2^{\circ}\right)$ on their respective maternally inherited chromosomes and hypermethylation (filled ovals) of these DMRs on the paternally inherited chromosomes, as seen in somatic cells, results in the balanced expression of growth-suppressing ncRNAs and growth-promoting protein-coding genes from these loci. 


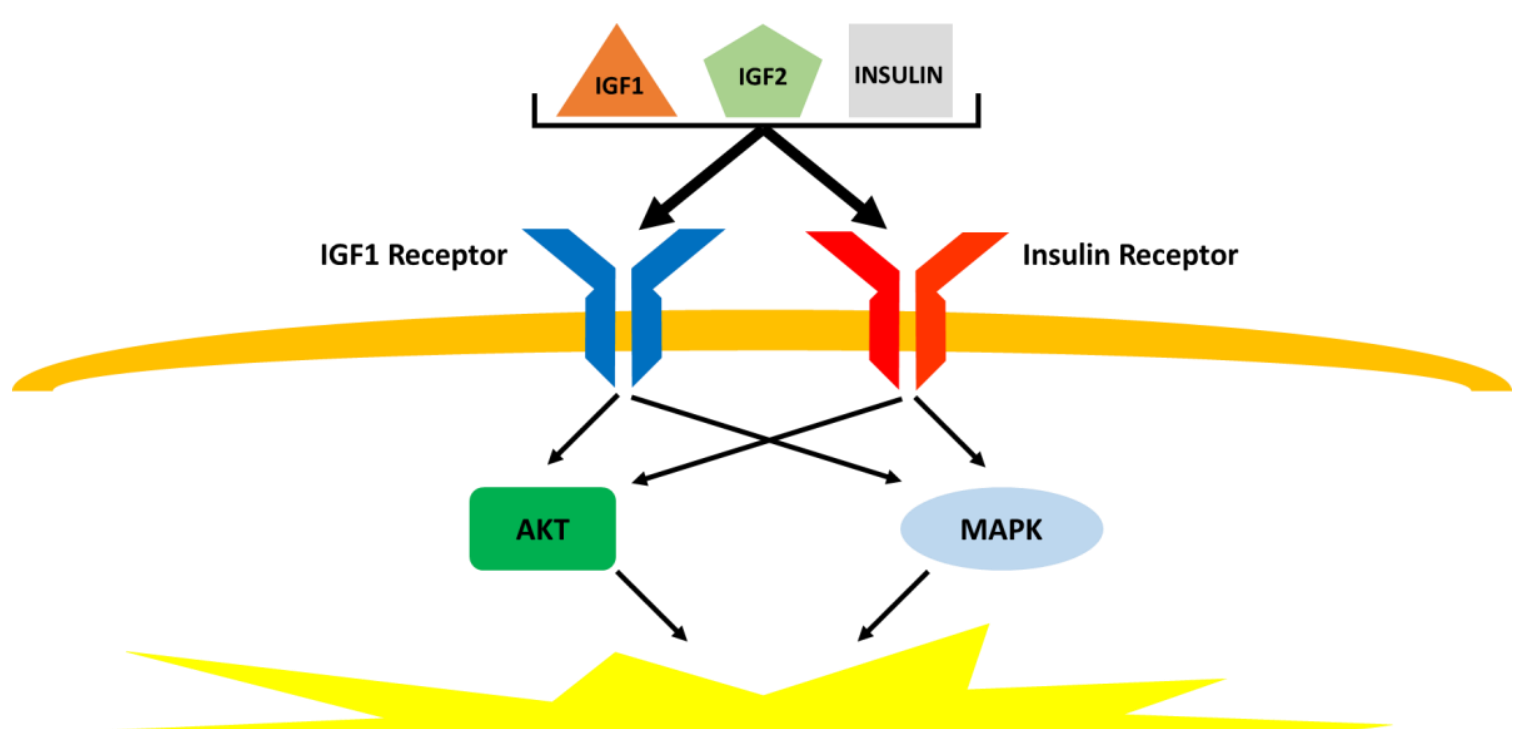

\section{Cell Survival and Proliferation}

\section{Figure 4. Insulin-like signaling}

Insulin-like signaling occurs via stimulation of the IGF1 receptor and insulin receptor by the ligands IGF1, IGF2, and insulin. 
A.

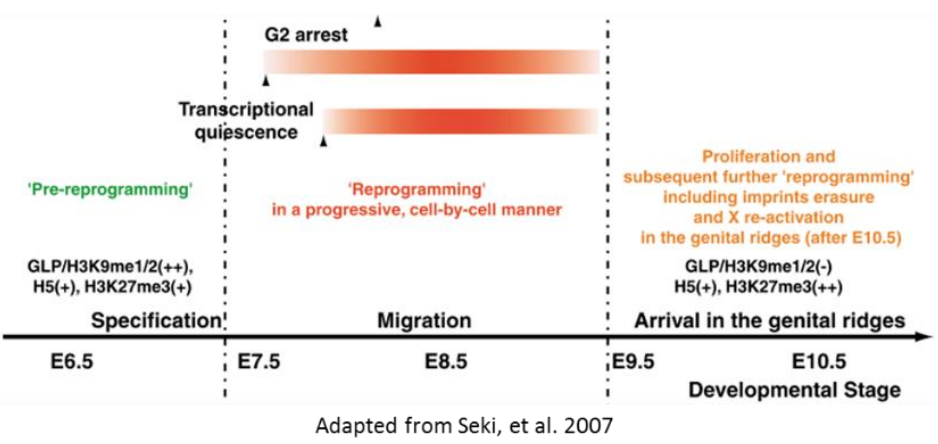

B.

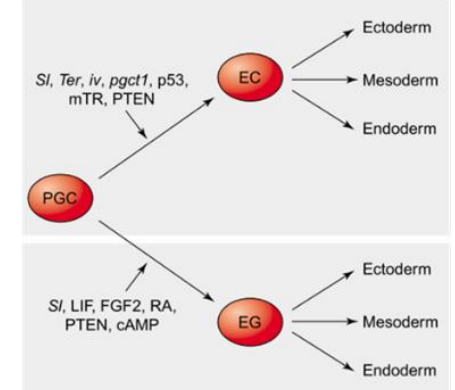

Adapted from Donovan and Miguel, 2003

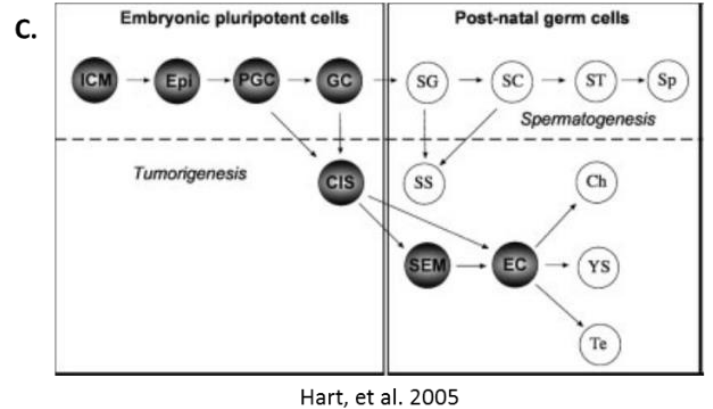

\section{Figure 5. Primordial germ cells and germ cell tumors}

(A) The epigenetic reprogramming of PGCs accompanies their migration and transcriptional quiescence. (B) Acquisition of pluripotency by PGCs can result in their transformation into EGCs or malignant EC cells. (C) EC cells are proposed to arise from germ cells and give rise to more differentiated TGCTs (ICM - inner cell mass; Epi epiblast; PGC - primordial germ cell; GC - gonocyte; SG - spermatogonial stem cell; SC - spermatocyte; ST - spermatid; Sp - spermatozoa; SS - spermatocytic seminoma; CIS carcinoma in situ; SEM - seminoma; EC - embryonal carcinoma; Ch - choriocarcinoma; YS - yolk sac; Te - teratoma) 


\section{Tumor heterogeneity and fluctuating cancer stem cell phenotypes}

It is well accepted that tumors are composed of heterogeneous tissues $[65,66]$. Among the subpopulations of cells proposed to exist within tumor tissues exists putative stem-like cell populations which exhibit enhanced capacity for malignancy. These CSC subpopulations are often identified based on their expression of certain surface antigens which also exist on normal stem cell populations, including SSEA4, CD133, and DLK1 [11, 67-69]. However, several studies have challenged the nature of true CSCs within tumors by providing evidence that these CSC-like subpopulations of cells dynamically express CSC-associated antigens [70, 71]. Notably, low-passage cell lines from human metastatic melanoma patients were recently shown to form tumors in vivo irrespective of their surface expression of the CSC marker CD133, and CD133-isolated subpopulations of these cells re-acquired CD133 expression during culture [70]. Indeed, this phenomenon has also been demonstrated in normal HSCs, where the expression of characteristic surface antigens was found to fluctuate over time [72]. Similarly, Chinese hamster ovary cells were used to demonstrate that the fluctuation of mRNA and protein expression can be coordinated with the cell cycle [73]. Interestingly, the expression dynamics observed in cells from tumor bulk and low-passage cell lines has also been observed in an established cell line, questioning the usefulness of these cells in tumor models [74].

Like bulk tumor tissues, cancer cell lines have also been assessed for their heterogeneity and the presence of CSC subpopulations. Unique CSC subpopulations have been identified in several cell lines which could provide insight into the development of treatment strategies that target CSCs $[68,75]$. However, also like bulk tumor tissues, the surface expression of CSC antigens was demonstrated to fluctuate in the established human 
ovarian carcinoma cell line A2780 [74]. Interestingly, in vitro expansion of single cells isolated from this cell line based on their expression of CD24 and CD44 demonstrated that $\mathrm{CD}^{-} 4^{-} \mathrm{CD} 44^{+}, \mathrm{CD}_{24}{ }^{+} \mathrm{CD} 44^{-}$, and even $\mathrm{CD} 24^{-} \mathrm{CD} 44^{-}$cells could give rise to $\mathrm{CD} 24^{+} \mathrm{CD} 44^{+}$ cells. Similarly, a recent investigation into B-cell acute lymphoblastic leukemia found that cell subpopulations isolated from a long-term culture system based on CD34 and CD38 surface expression and expanded in vitro resulted in the re-establishment of the expression phenotypes found in the initial cell population, and similar results were obtained when expanding single cells isolated from each subpopulation [71]. Considering that EC cells are proposed to be the CSCs which may give rise to GCTs, and EC cells exhibit pluripotent characteristics related to their expression of the imprinted $H 19$ gene, investigations into the phenotypic fluctuations of an EC cell line at the protein and mRNA levels may provide new insight into the mechanisms by which this occurs, and whether the expression of imprinted genes is related to this phenomenon $[18,43]$.

The human EC cell line NTera2 was derived from the teratocarcinoma cell line Tera2, which originated from a lung metastasis of teratocarcinoma in a 22-year-old male [76]. Among the cells observed from a nude mouse xenograft of Tera2, EC-like cells were found which resembled certain cells noted in early passages of Tera2 [77]. As an established EC cell line derived from this xenograft, NTera2 thus exemplifies the heterogeneity of cancerous tissues. Similarly, as the pluripotent stem-like cells of teratocarcinomas which may give rise to other TGCTs, EC cells such as NTera2 represent an interesting model for studying the nature of putative CSC populations [43]. Interestingly, HSCs are known to express CD133 and VSELs express CD133 and SSEA4, and both of these CSC-associated antigens have been found on the surface of EC cells [78- 
81]. Notably, SSEA4 is a pluripotent stem cell marker and has also been used to identify pluripotent stem-like cells within primary EC tissues [69]. Though the mechanisms by which these antigens exert their stem-like effects are largely uncharacterized, CD133 and SSEA4 represent antigens shared by cells of prospective PGC origins and are thus intriguing candidates for investigating the existence of CSCs within NTera2 as an established cell line. 


\section{CHAPTER 2: MATERIALS AND METHODS}

\section{Cell culture}

The NTera2 cell line was purchased from the American Type Culture Collection (ATCC) and ATCC instructions were followed for culturing of NTera2 cells. Cells were maintained at a high density in a media consisting of Dulbecco's Modified Eagle's Medium (DMEM)

with high glucose $(4,500 \mathrm{mg} / \mathrm{mL})$ supplemented with $10 \%$ fetal bovine serum, $100 \mathrm{U} / \mathrm{mL}$ penicillin, and $10 \mu \mathrm{g} / \mathrm{mL}$ streptomycin, and the cells were split 1:3 upon reaching confluency.

\section{Isolation of mononuclear cells from human umbilical cord blood}

Human umbilical cord blood was provided by the Cleveland Cord Blood Center (Cleveland, $\mathrm{OH}$ ) and centrifuged at $400 \mathrm{x} \mathrm{g}$ for 30 minutes in Ficoll-Paque Plus (GE Healthcare). Low-density mononuclear cells were isolated based on the resulting gradient following centrifugation.

\section{AML patient samples}

Forty-five newly diagnosed patients with acute non-promyelocytic leukemia were enrolled in a study and treated at the University of Bialystok Hematology Department (Bialystok, Poland) from 2008 to 2016. Patient information is listed in Table 1. The World Health Organization (WHO) classification system was used for diagnosing patients [82]. A sevenday induction therapy following the DAC schedule was used to treat patients: seven 
consecutive days of $200 \mathrm{mg} / \mathrm{m}^{2}$ cytarabine via continuous IV infusion, three consecutive days of $50 \mathrm{mg} / \mathrm{m}^{2}$ anthracycline via IV push; five days of $5 \mathrm{mg} / \mathrm{m}^{2}$ cladribine via IV push [83]. Following evaluation of the induction response, non-responding patients were given re-induction protocol therapy of cytarabine, cladribine, filgrastim, and mitoxantrone (CLAG-M) and/or idarubicin, cytarabine, and etoposide (ICE) [84-86]. Consolidation therapies for patients who achieved complete remission consisted of cytarabine and mitoxantrone first, then a high dose of cytarabine, and finally either allogenic HSC transplantation or maintenance therapy.

\section{Testicular germ cell tumor tissues}

Formalin-fixed paraffin-embedded (FFPE) human TGCT tissues were generously provided as a gift from Joanna Reszec at the Medical University of Bialystok (Bialystok, Poland). Pathology slides were also provided for each tumor which identified the EC component of the tissue samples.

\section{Combined bisulfite-restriction analysis and bisulfite sequencing of genomic DNA}

For NTera2 cells and all MNCs, genomic DNA (gDNA) was isolated from cells using the DNeasy Blood and Tissue Kit (Qiagen) and was subjected to bisulfite treatment using the EpiTect Bisulfite Kit (Qiagen). For FFPE tissues, the Epitect Fast FFPE Bisulfite Kit (Qiagen) was used to obtain bisulfite-treated gDNA (BSgDNA) from the EC component of FFPE tissue. For AML samples, the DNA Mini Kit (Qiagen) was used to isolate gDNA from whole peripheral blood leukocytes. The amplification of DNA sequences was performed using DNA oligo primer pairs specific to BSgDNA with the following 
thermocycler conditions: for AmpliTaq DNA Polymerase (Applied Biosystems) $-\left(95^{\circ} \mathrm{C}\right.$ for 2 minutes, annealing temperature for 1 minute, $72^{\circ} \mathrm{C}$ for 1 minute) $x 1 \mathrm{cycle},\left(95^{\circ} \mathrm{C}\right.$ for 30 seconds, annealing temperature for 1 minute, $72^{\circ} \mathrm{C}$ for 1 minute $) \times$ cycle number, $\left(72^{\circ} \mathrm{C}\right.$ for 10 minutes) x 1 cycle; for AmpliTaq Gold DNA Polymerase (Applied Biosystems) $\left(95^{\circ} \mathrm{C}\right.$ for 8 minutes $) \times 1$ cycle, $\left(95^{\circ} \mathrm{C}\right.$ for 2 minutes, annealing temperature for 1 minute, $72^{\circ} \mathrm{C}$ for 1 minute) $\mathrm{x} 2 \mathrm{cycles},\left(95^{\circ} \mathrm{C}\right.$ for 30 seconds, annealing temperature for 1 minute, $72^{\circ} \mathrm{C}$ for 1 minute) $\mathrm{x}$ cycle number, $\left(72^{\circ} \mathrm{C}\right.$ for 10 minutes $) \times 1$ cycle. Primer sequences for each locus are listed in Table 2. The annealing temperatures and cycle numbers for each amplicon are listed in Table 3.

Combined bisulfite-restriction analysis (COBRA) was carried out on each amplicon via restriction enzyme digestion using either TaqI or BstUI (New England Biolabs) with the following thermocycler conditions: for BstUI $-60^{\circ} \mathrm{C}$ for 2 hours; for TaqI $-65^{\circ} \mathrm{C}$ for 2 hours. The digested amplicons were separated by electrophoresis in agarose gels, and densitometric analysis of ethidium bromide-labeled bands was performed on photographed agarose gels using ImageJ software (National Institutes of Health). The specific COBRA conditions for each amplicon are listed in Table 3.

Bisulfite sequencing was performed by ligating amplicons into TOPO vectors (pCR2.1 for DLK1-MEG3 IG-DMR and pCR4 for IGF2-H19 ICR) (Invitrogen) and transforming TOP10 bacteria using the TOPO TA Cloning Kit (Invitrogen). Plasmids were purified from several colonies using the QIAprep Spin Miniprep Kit (Qiagen) and sequenced using the M13 primer pair. Sequencing results were compiled for methylation analysis using CpGviewer software (University of Leeds, UK). 


\section{Real-time quantitative PCR}

Extraction of RNA from NTera2 cells and MNCs (for comparison with NTera2 cells) was performed using the RNeasy Mini Kit (Qiagen). Human testes mixed tissue RNA was purchased separately (Ambion). For AML samples, total RNA was first purified using TRIZOL (Life Technologies) before diluting it with water to a final concentration of 1000$2500 \mathrm{ng} / \mu \mathrm{L}$, verifying its quality using a Nanodrop spectrophotometer (ThermoFisher) using a A260/280 threshold of 1.6. For MNCs used in experiments with AML samples, RNA was extracted using TRIZOL (Life technologies). For RNA isolated from NTera2 and MNCs and purchased for human testes, the First Strand cDNA Synthesis Kit (Thermo Fisher) was employed for preparation of cDNA from $200 \mathrm{ng}$ RNA in $10 \mu \mathrm{L}$ reaction volumes. For RNA isolated from AML patients, $2500 \mathrm{ng}$ of RNA was transcribed to cDNA using Superscript VILO (Life Technologies). For MNCs used in experiments with AML samples, 1000 ng of RNA was transcribed to cDNA using the First Strand cDNA synthesis kit without the addition of oligodT to the reaction mixture. Gene expression analysis was carried out at least twice in duplicate using $2 \mathrm{ng}$ of cDNA, $12.5 \mu \mathrm{L}$ SYBR Select Master Mix (Applied Biosystems), and $150 \mathrm{nM}$ forward and reverse primer sequences in $25 \mu \mathrm{L}$ reaction mixtures. Real-time quantitative PCR (RT-qPCR) for each reaction mixture was performed using a 7500 Fast Real-Time PCR system (Applied Biosystems) with the following amplification conditions: $50^{\circ} \mathrm{C}$ for 20 seconds; $95^{\circ} \mathrm{C}$ for 10 minutes; at least 45 cycles repeating the two steps $95^{\circ} \mathrm{C}$ for 15 seconds then $60^{\circ} \mathrm{C}$ for 1 minute; $95^{\circ} \mathrm{C}$ for 15 seconds; $60^{\circ} \mathrm{C}$ for 1 minute; $95^{\circ} \mathrm{C}$ for 30 seconds; then $60^{\circ} \mathrm{C}$ for 15 seconds. The $\Delta \Delta \mathrm{Ct}$ method was used to quantify RT-qPCR results. Primer sequences for each gene are listed in Table 4. 


\section{NTera2 treatment with 5-aza-2'-deoxycytidine}

For proliferation assays of NTera2 cells treated with 5-aza-2'-deoxycytidine (5-azaD; Sigma), cells were plated at a density of 10,000 cells/well on 12-well plates (Greiner). Wells were washed with PBS, trypsonized, and counted in triplicate on each day. Cells were washed with PBS every day prior to adding fresh media supplemented with either vehicle (DMSO) for control cells or concentrations of 5-azaD in DMSO beginning on Day 0. Proliferation assays were repeated twice in triplicate.

For DNA and RNA isolation from NTera2 cells treated with 5-azaD, cells were plated at a density of 150,000 cells/plate on $10-\mathrm{cm}$ plates (Greiner). Cells were washed with PBS every day prior to adding fresh media supplemented with either vehicle (DMSO) for control cells or $100 \mathrm{nM}$ 5-azaD in DMSO beginning on Day 0. Individual plates were trypsonized for each treatment either on Day 3 or Day 7 and the collected cells were divided in half for DNA and RNA isolation, which was later performed using the DNeasy Blood and Tissue Kit (Qiagen) and RNeasy Mini Kit (Qiagen), respectively.

\section{Plasmid preparation and transfection of NTera2 cells}

Stably transfecting NTera2 cells with short hairpin RNA (shRNA)-generating plasmids was performed using the pSUPER.retro.puro plasmid (Oligoengine). Plasmid preparation was performed according to the manufacturer's instructions utilizing shRNAs against DLK1 or the control gene Renilla. Target sequences for shRNA constructs are listed in Table 5. NTera2 cells were electroporated for $2.5 \mathrm{~ms}$ at $125 \mathrm{~V}$ and selection of transfected cells was performed using $140 \mathrm{ng} / \mathrm{mL}$ puromycin. 


\section{Proliferation assays of transfected NTera2 cells}

Cells were plated at a density of 10,000 cells/well on 12-well plates (Greiner). Wells were washed with PBS, trypsonized, and counted in triplicate on every second day beginning on Day 0. Fresh media was exchanged for each well on every second day beginning on Day 0 after an initial PBS wash on Day 0 only. Proliferation assays were repeated twice in triplicate.

\section{Tumor growth in immunodeficient mice}

All experiments in severe combined immunodeficiency mice (SCID) were performed in adherence to the guidelines established by the Association for the Assessment and Accredidation of Laboratory Animal Care at the University of Louisville upon approval by The Institutional Animal Care and Use Committee. SCID mice of 6-9 weeks of age were irradiated with $350 \mathrm{cGy}$ and injected subcutaneously with 8 million stably transfected NTera 2 cells in $200 \mu \mathrm{L}$ volume of 50\% Matrigel Matrix (Corning) in PBS at 24 hours postirradiation. Mice were monitored for signs of illness before being sacrificed after 5.5 weeks post-irradiation. The tumors were resected and measured using a digital caliper, then fixed via overnight formalin treatment followed by washing and storage in $70 \%$ ethanol.

\section{Organ seeding efficiencies}

The liver, lungs, and bone marrow were harvested from each mouse at the time of sacrifice. Isolation of gDNA from each tissue was performed using the DNeasy Blood and Tissue Kit (Qiagen). Evaluation of human-murine chimerism was achieved by qPCR analysis of 100 ng gDNA using primers targeting a human $\alpha$-satellite sequence and a murine $\beta$-actin 
sequence on a 7500 Fast Real-Time PCR system (Applied Biosystems). Reaction mixtures consisting of $100 \mathrm{ng}$ gDNA, $60 \mathrm{nM}$ forward and reverse primers (Table 4), and $12.5 \mu \mathrm{L}$ SYBR Select Master Mix (Applied Biosystems) in $25 \mu \mathrm{L}$ reaction volumes were analyzed by qPCR at least once in duplicate with the following amplification conditions: $50^{\circ} \mathrm{C}$ for 20 seconds; $95^{\circ} \mathrm{C}$ for 10 minutes; at least 45 cycles repeating the two steps $95^{\circ} \mathrm{C}$ for 15 seconds then $60^{\circ} \mathrm{C}$ for 1 minute; $95^{\circ} \mathrm{C}$ for 15 seconds; $60^{\circ} \mathrm{C}$ for 1 minute; $95^{\circ} \mathrm{C}$ for 30 seconds; then $60^{\circ} \mathrm{C}$ for 15 seconds. The difference in $\mathrm{Ct}$ values between the human $\alpha$ satellite sequence and murine $\beta$-actin for each sample generated $\Delta \mathrm{Ct}$ values for comparison to a standard curve of serially diluted NTera2 cells in a fixed number of murine MNCs.

For stably transfected cell lines, organs were harvested from the same mice from which tumors were isolated. For NTera2 subpopulations, SCID mice of 2-4 months of age were irradiated with $350 \mathrm{cGy}$, and intravenous injections of $100 \mu \mathrm{L}$ cell suspensions containing 100,000 freshly sorted NTera2 subpopulation cells (CD133-SSEA4 ${ }^{-}, \mathrm{CD}^{-} 33^{-}$ $\mathrm{SSEA}^{+}, \mathrm{CD} 133^{+} \mathrm{SSEA}^{-}$, and $\mathrm{CD} 133^{+} \mathrm{SSEA} 4^{+}$) in phosphate-buffered saline were performed at 24 hours post-irradiation.

\section{Proliferation assays of NTera2 cells treated with insulin-like growth factors}

Cells were plated at a density of 10,000 cells/well on 12-well plates (Greiner). Wells were washed with PBS, trypsonized, and counted in triplicate on each day. Cells were washed with PBS every day prior to adding fresh media supplemented with either 10\% FBS, $0.5 \%$ BSA, or growth factors (10 ng/mL IGF1, $10 \mathrm{ng} / \mathrm{mL}$ IGF2, and/or $10 \mu \mathrm{g} / \mathrm{mL}$ insulin) in 0.5\% BSA beginning on Day 0. Proliferation assays were repeated twice in triplicate. 


\section{Western blot}

NTera2 cells were plated onto 6-well plates (Greiner) at a density of 350,000 cells/well. Following quiescence in $0.5 \%$ BSA media, cells were washed with PBS and $1 \mathrm{~mL}$ of $0.5 \%$ BSA alone or supplemented with growth factors $(10 \mathrm{ng} / \mathrm{mL}$ IGF1, $10 \mathrm{ng} / \mathrm{mL}$ IGF2, or 10 $\mu \mathrm{g} / \mathrm{mL}$ insulin) was added to the cells. Following a 10 minute incubation at $37^{\circ} \mathrm{C}$ and $5 \%$ humidity, cells were washed again with PBS before adding RIPA buffer (Santa Cruz Biotechnology) containing $10 \mu \mathrm{L} / \mathrm{mL}$ protease inhibitors (Sigma), $1 \mathrm{mM}$ sodium orthovanadate, and $10 \mathrm{mM}$ sodium fluoride for a 30 minute incubation on ice. The resulting lysate was collected and centrifuged at $10,000 \times \mathrm{g}$ for 15 minutes at $4^{\circ} \mathrm{C}$. Protein concentrations were determined for each sample using the BCA Protein Assay Kit (Pierce). $20 \mu \mathrm{g}$ was separated on NuPAGE (Invitrogen) tris-glycine gels before transferring to PVDF membranes for 60 minutes at $100 \mathrm{~V}$. Membranes blocked for one hour at room temperature in a $2.5 \%$ BSA in TBS-T (20 mM tris $\mathrm{pH} 7.5,150 \mathrm{mM} \mathrm{NaCl}, 0.1 \%$ Tween 20 ) before overnight incubation at $4^{\circ} \mathrm{C}$ with primary phospho-AKT or phospho-MAPK antibodies (Cell Signaling) in 2.5\% BSA in TBS-T. Membranes were washed in TBS-T and the secondary antibody (Cell Signaling) in 2.5\% BSA in TBS-T was added for a 1 hour incubation at room temperature. Membranes were washed with TBS-T before developing with ECL reagents (Amersham).

\section{Immunofluorescence}

Ntera 2 cells were plated on $35 \mathrm{~mm}$ Fluorodish glass slides (World Precision Instruments) at a density of 50,000 cells/slide. Cells were washed with PBS and fixed via incubation in 3.7\% paraformaldehyde in PBS for 20 minutes at room temperature. Cells were washed 
with PBS, incubated in $0.1 \%$ triton in PBS for 1 minute at room temperature, and washed again with PBS before blocking for 2 hours in $2.5 \% \mathrm{BSA}$ in PBS at $37^{\circ} \mathrm{C}$. Cells were incubated overnight at $4^{\circ} \mathrm{C}$ with 1:250 mouse anti-human DLK1 antibody (Abcam), then incubated for 1.5 hours at $37^{\circ} \mathrm{C}$ in the dark with goat anti-mouse secondary antibody (Invitrogen) conjugated to Alexa Fluor 594. A 1.5 hour incubation with Alexa Fluor 488 phalloidin (Invitrogen) at $37^{\circ} \mathrm{C}$ in the dark followed before the cells were stained with 4',6diamidino-2-phenylindole (DAPI; Life Technologies) at room temperature for 15 minutes. Confocal microscopy of stained cells was performed using a FV100-IX81 confocal microscope (Olympus) with Fluoview version 4.1a software (Olympus).

\section{Flow cytometry analysis of the IGF1 receptor and insulin receptor}

For INSR, NTera2 cells were detached using Cell Stripper (Corning) and incubated at $37^{\circ} \mathrm{C}$ and $5 \%$ humidity in $0.5 \%$ BSA media for two hours, then labeled with goat anti-human INSR-APC (R\&D Systems) or goat IgG APC control (R\&D Systems) and incubated in the dark at $37^{\circ} \mathrm{C}$ for 30 minutes. Cells were washed and resuspended in $0.5 \%$ BSA media for analysis using a LSR II flow cytometer (BD Biosciences) and FACSDIVA (BD Bioscences) software.

For IGF1R, NTera2 cells were detached using Cell Stripper (Corning) and incubated at $37^{\circ} \mathrm{C}$ and $5 \%$ humidity in $0.5 \%$ BSA media for two hours, then labeled with chicken anti-human IGF1R primary antibody (Abcam). Control cells were not labeled with a primary antibody. Following a 30 minute incubation in the dark at $37^{\circ} \mathrm{C}$, cells were washed and resuspended with $0.5 \%$ BSA media and a rabbit anti-chicken FITC conjugated secondary antibody (Abcam) was added to experiment and control cells. Cells were 
incubated for 30 minutes in the dark at $37^{\circ} \mathrm{C}$, washed and resuspended in $0.5 \%$ BSA media, and analyzed by flow cytometer.

\section{Fluorescence-activated cell sorting}

NTera2 cells were detached using Cell Stripper (Corning) and incubated in DMEM with $2 \% \mathrm{FBS}$ for 2 hours at $37^{\circ} \mathrm{C}$ and $5 \%$ humidity, then labeled with mouse anti-human CD133APC (Miltenyi Biotec) and mouse anti-human SSEA4-PE (eBioscience) antibodies in the dark for 30 minutes at room temperature. Mouse IgG1-APC (Miltenyi Biotec) and mouse IgG3-PE (eBioscience) were used as isotype controls. Cells were washed and resuspended in $2 \%$ FBS media before sorting by CD133 and SSEA4 expression using a Moflo XDP cell sorter (Beckman Coulter).

\section{Chemotaxis assay}

NTera2 cells freshly sorted by CD133 and SSEA4 surface expression were incubated in DMEM with $0.5 \%$ BSA for 4 hours at $37^{\circ} \mathrm{C}$ and $5 \%$ humidity, then employed in chemotaxis assays using modified Boyden chambers. Each $8 \mu \mathrm{m}$ Transwell insert (Corning) was incubated with $1 \%$ gelatin for 60 minutes before removing the gelatin and loading 40,000 cells into the upper chamber. Lower chambers contained DMEM with either $0.5 \%$ BSA as a negative control or $10 \%$ FBS. Following a 40 -hour incubation at $37^{\circ} \mathrm{C}$ and $5 \%$ humidity, the PROTOCOL Hema3 stain set (Fisher Scientific) was used to stain cells on each insert. Migrated cells were counted under an inverted microscope after removing non-migrated cells from the top of each insert with a cotton swab. 


\section{Single-cell expansion and FACS analysis}

Single NTera2 cells were sorted into 96-well plates (Greiner) by their CD133 and SSEA4 expression and cultured under normal conditions. Cells which expanded into colonies were transferred to larger diameter wells upon reaching confluency. The surface expression analysis of CD133 and SSEA4 by expanded cells was carried out in a manner similar to the initial sorting strategy.

\section{Statistical analysis}

All statistical analyses were carried out using the Mann-Whitney U Test unless otherwise noted. Graphpad Prism software was used for all statistical analyses and graph preparation. Data are graphed either as means \pm SEM or in survival format, and $\mathrm{p}<0.05$ is considered significant with $* \mathrm{p}<0.05, * * \mathrm{p}<0.01$, and $* * * \mathrm{p}<0.001$ 


\begin{tabular}{|c|c|c|c|}
\hline \multicolumn{2}{|r|}{ Parameter } & Characteristic & Value \\
\hline \multirow{5}{*}{\multicolumn{2}{|c|}{ General Information }} & Number of patients & 45 \\
\hline & & Mean (range) age in years & $46.5(18-64)$ \\
\hline & & Mean $( \pm \mathrm{SD})$ white blood cell count $(\mathrm{G} / \mathrm{l})$ & $51.41 \pm 28.04$ \\
\hline & & Mean (range) of blastic cells in the peripheral blood & $56(0-97)$ \\
\hline & & Mean (range) of blastic cells in the bone marrow & $65(20-97)$ \\
\hline \multirow{13}{*}{$\begin{array}{l}\text { AML Subtypes based } \\
\text { on WHO classification } \\
(\mathrm{n},(\%))\end{array}$} & \multirow[t]{3}{*}{ AML with recurring genetic abnormalities } & $\mathrm{t}(8 ; 21)(\mathrm{q} 22 ; \mathrm{q} 22) ;(\mathrm{AML} 1 / \mathrm{ETO})$ & $4(8.9 \%)$ \\
\hline & & 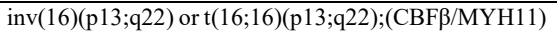 & $1(2.2 \%)$ \\
\hline & & $\mathrm{t}(9 ; 11) ;$ MLLT3-MLL & $3(6.7 \%)$ \\
\hline & \multicolumn{2}{|c|}{ AML with multilineage dysplasia without antecedent MDS } & $2(4.4 \%)$ \\
\hline & \multicolumn{2}{|l|}{ AML (therapy-related) } & $0(0 \%)$ \\
\hline & \multirow[t]{8}{*}{ AML (not otherwise categorized, $\mathrm{n}=35$ ) } & AML (minimally differentiated) & $4(8.9 \%)$ \\
\hline & & AML (without maturation) & $8(17.8 \%)$ \\
\hline & & AML (with maturation) & $13(28.9 \%)$ \\
\hline & & Acute myelomonocytic leukemia (AMMoL) & $8(17.8 \%)$ \\
\hline & & AMMoL with eosinophilia & $0(0.0 \%)$ \\
\hline & & Acute monocytic leukemia & $2(4.4 \%)$ \\
\hline & & Acute erythroid leukemia & $0(0.0 \%)$ \\
\hline & & Acute megakaryoblastic leukemia & $0(0.0 \%)$ \\
\hline \multicolumn{2}{|l|}{ Mutations } & $F L T 3-I T D / N M P 1_{m u t} / C E B P A_{m u t}$ & $8 / 2 / 1$ \\
\hline \multirow{4}{*}{$\begin{array}{l}\text { Induction } \\
\text { Outcome }\end{array}$} & & Complete remission after 1 st induction & 24 \\
\hline & & Complete remission after 2nd induction & 6 \\
\hline & & Complete remission after 3rd induction & 3 \\
\hline & & Mortality (1st/2nd/3rd induction/consolidation) & $5 / 4 / 3 / 0$ \\
\hline \multirow{2}{*}{\multicolumn{2}{|c|}{ Post-consolidation Treatment }} & Allogenic hematopoietic stem cell transplant & 30 \\
\hline & & Maintenance & 3 \\
\hline \multirow{4}{*}{\multicolumn{2}{|c|}{ Risk }} & Favorable risk & $7(15.6 \%)$ \\
\hline & & Intermediate risk I & $10(22.2 \%)$ \\
\hline & & Intermediate risk II & $8(17.8 \%)$ \\
\hline & & Unfavorable risk & $20(44.4 \%)$ \\
\hline
\end{tabular}

Table 1. AML patient information $\left(N P M 1_{m u t}\right.$ - mutated nucleophosmin; CEBPA $A_{m u t}$ mutated core binding factor leukemia; FLT3-ITD = internal tandem duplication of FMSlike tyrosine kinase 3) 


\begin{tabular}{|c|c|c|}
\hline Locus & 1st PCR Primer Pair (5'-3') & 2nd PCR Primer Pair (5'-3') \\
\hline IGF2-H19 ICR & $\begin{array}{l}\text { F - AGGTGTTTTAGTTTTATGGATGATGG } \\
\text { R - TCCCATAAATATCCTATTCCCAAATAACC }\end{array}$ & $\begin{array}{l}\text { F - TGTATAGTATATGGGTATTTTTGGAGGTTT } \\
\text { R - TCCCATAAATATCCTATTCCCAAATAACC }\end{array}$ \\
\hline$D L K 1-M E G 3$ IG-DMR (CG6) & $\begin{array}{l}\text { F - TGGGAATTGGGGTATTGTTTATAT } \\
\text { R - AAACAATTTAACAACAACTTTCCTC }\end{array}$ & $\begin{array}{l}\text { F - GTTAAGAGTTTGTGGATTTGTGAGAAATG } \\
\text { R - CTAAAAATCACCAAAACCCATAAAATCAC }\end{array}$ \\
\hline MEG3 DMR (CG7) & $\begin{array}{l}\text { F- TTATTTTTTTTGAATAATAAGAGAAAGTATG } \\
\text { R - CTCATTTCTCTAAAAATAATTAACC }\end{array}$ & $\begin{array}{l}\text { F - TTATTTTTTTGAATAATAAGAGAAAGTATG } \\
\text { R - CCCCAAATTCTATAACAAATTACT }\end{array}$ \\
\hline MEG3 Promotor (CG9) & $\begin{array}{l}\text { F - TGAGGAAGTAGGGGTTTATAGAGAG } \\
\text { R - AACCCTACAACCCCACAAAA }\end{array}$ & $\begin{array}{l}\text { F - TGAGGAAGTAGGGGTTTATAGAGAG } \\
\text { R - TTCACAATAAACCCCACTCTCC } \\
\end{array}$ \\
\hline MEG3 Intragenic (CG8) & $\begin{array}{l}\text { F - GTTTGAGATTTGTTGGGTATTT } \\
\text { R - CGTTATTTTGGGTTTTGAGTTG }\end{array}$ & $\begin{array}{l}\text { F - GTTTGAGATTTGTTGGGTATTT } \\
\text { R - CGTTATTTTGGGTTTTGAGTTG }\end{array}$ \\
\hline ZAC DMR & $\begin{array}{l}\text { F- GGGGTAGTYGTGTTTATAGTTTAGTA } \\
\text { R - CRAACACCCAAACACCTACCCTA }\end{array}$ & $\begin{array}{l}\text { F - GGGGTAGTYGTGTTTATAGTTTAGTA } \\
\text { R - CRAACACCCAAACACCTACCCTA }\end{array}$ \\
\hline P57-LITI DMR & $\begin{array}{l}\text { F - TTTTGGTAGGATTTTGTTGAGGAGT } \\
\text { R - CCTCACACCCAACCAATACCTC }\end{array}$ & $\begin{array}{l}\text { F - TTTTGGTAGGATTTTGTTGAGGAGT } \\
\text { R - CCTCACACCCAACCAATACCTC }\end{array}$ \\
\hline SNRPN DMR & $\begin{array}{l}\text { F - GTGTTGTGGGGTTTTAGGGGTTTAG } \\
\text { R - CTCCCCAAACTATCTCTTAAAAAAAACC }\end{array}$ & $\begin{array}{l}\text { F - AGGGAGTTGGGATTTTTGTATTG } \\
\text { R - CTCCCCAAACTATCTCTTAAAAAAAACC }\end{array}$ \\
\hline PEGl DMR & $\begin{array}{l}\text { F - TTGTTGGTTAGTTTTGTAYGGTT } \\
\text { R - AAAAATAACACCCCCTCCTCAAAT }\end{array}$ & $\begin{array}{l}\text { F - TTGTTGGTTAGTTTTGTAYGGTT } \\
\text { R - CCCAAAAACAACCCCAACTC }\end{array}$ \\
\hline DNMT1 DMR & $\begin{array}{l}\text { F- GTTTAGTTTTTAGTTATTAGGGAGTTA } \\
\text { R - ACTCCRTTCCATCCTTCTACAC } \\
\end{array}$ & \begin{tabular}{|l|} 
F - TTTTGTTTGTTTTTTTGAGTT \\
R - ACTCCRTTCCATCCTTCTACAC \\
\end{tabular} \\
\hline PEG10 DMR & $\begin{array}{l}\text { F - TTGGTTTAGGTGTGGGATTTT } \\
\text { R - AAACATTCTAAAATACTACTCCATCTC }\end{array}$ & $\begin{array}{l}\text { F - TTGGTTTAGGTGTGGGATTTT } \\
\text { R - AAACATTCTAAAATACTACTCCATCTC }\end{array}$ \\
\hline TRAPPC9 DMR & $\begin{array}{l}\text { F - GGTTTTAGTAGTATTAGGTA } \\
\text { R - AAACTCTTTACCCTATAAAT }\end{array}$ & \begin{tabular}{|l|} 
F - GGTTTTAGTAGTATTAGGTA \\
R - AAACTCTTTACCCTATAAAT \\
\end{tabular} \\
\hline L3MBTL DMR & $\begin{array}{l}\text { F - TATGAGGCGAAGAGAGGGTTATGGTAT } \\
\text { R - AAAACCCAACTCAAAACCTAAAAAAC } \\
\end{array}$ & $\begin{array}{l}\text { F - TATGAGGCGAAGAGAGGGTTATGGTAT } \\
\text { R - AAAACCCAACTCAAAACCTAAAAAAC } \\
\end{array}$ \\
\hline PEG3 DMR & $\begin{array}{l}\text { F - AAAAGGTATTAATTATTTATAGTTTGGT } \\
\text { R - AAAAATATCCACCCTAAACTAATAA }\end{array}$ & $\begin{array}{l}\text { F - AAAAGGTATTAATTATTTATAGTTTGGT } \\
\text { R - AAAAATATCCACCCTAAACTAATAA }\end{array}$ \\
\hline
\end{tabular}

Table 2. Primer list for PCR of BSgDNA 


\begin{tabular}{|c|c|c|c|c|c|c|c|}
\hline \multirow[b]{2}{*}{ Locus } & \multicolumn{3}{|c|}{ 1st PCR Conditions } & \multicolumn{3}{|c|}{ 2nd PCR Conditions } & \multirow{2}{*}{\begin{tabular}{|l|} 
COBRA \\
Restriction \\
Enzyme
\end{tabular}} \\
\hline & Polymerase & \begin{tabular}{|l|} 
Annealing \\
Temperature \\
$\left({ }^{\circ} \mathrm{C}\right)$ \\
\end{tabular} & Cycles & Polymerase & $\begin{array}{l}\text { Annealing } \\
\text { Temperature } \\
\left({ }^{\circ} \mathrm{C}\right) \\
\end{array}$ & Cycles & \\
\hline $\begin{array}{l}\text { DLK1-MEG3 IG-DMR } \\
\text { (CG6) }\end{array}$ & AmpliTaq & 55 & 35 & AmpliTaq & 55 & 35 & BstUI \\
\hline MEG3 DMR (CG7) & GoldTaq & 57 & 40 & GoldTaq & 57 & 40 & TaqI \\
\hline MEG3 Intragenic (CG8) & AmpliTaq & 55 & 35 & AmpliTaq & 55 & 35 & BstUI \\
\hline ZAC DMR & GoldTaq & 55 & 35 & GoldTaq & 55 & 38 & BstUI \\
\hline P57-LIT1 DMR & GoldTaq & 55 & 38 & GoldTaq & 55 & 58 & TaqI \\
\hline SNRPN DMR & AmpliTaq & 60 & 35 & AmpliTaq & 60 & 35 & BstUI \\
\hline PEG1 DMR & AmpliTaq & 55 & 35 & GoldTaq & 55 & 38 & TaqI \\
\hline L3MBTL DMR & GoldTaq & 55 & 35 & AmpliTaq & 55 & 35 & TaqI \\
\hline PEG3 DMR & AmpliTaq & 55 & 35 & AmpliTaq & 55 & 35 & BstUI \\
\hline
\end{tabular}

Table 3. PCR conditions for amplifying BSgDNA 


\begin{tabular}{|l|l|l|}
\hline Gene & Forward Primer $\left(\mathbf{5}^{\prime}\right.$-3') & Reverse Primer $\mathbf{( 5 '}$-3') \\
\hline$I G F 2$ & CCTCCTGGAGACGTACTGTGCT & TGGACTGCTTCCAGGTGTCATA \\
\hline$H 19$ & TGACAAGCAGGACATGACATGG & CAGCCTAAGGTGTTCAGGAAGG \\
\hline$D L K 1$ & GCGAGGATGACAATGTTTGCA & GGTTCTCCACAGAGTCCGTGAA \\
\hline$I G F I R$ & ATCCCGGACCCAAGTCTTCT & CCACATTCGAGGTCCCTTCC \\
\hline$I N S R$ & GCACCATCTTCAAGGGCAATTTG & AGGAAGGACAAGGAGACCAAGG \\
\hline$I G F 1$ & TCAAAACGAGGCCCGAAGAT & GAGCCCATAGACCCGGAAGA \\
\hline OCT4 & GATGCACACCATGTCCTCCT & AAAAGCCCCTGTCTCCACAC \\
\hline$T D G F 1$ & GCTGGATGTCAGGGCTCTTT & TCAAGAGATTTATCGAGCACCTTCT \\
\hline$M Y C$ & ACATGTAATTCTACCAAGGTCTTCT & AGGTCCACATTAGAATTAGTCTCCA \\
\hline$P 57$ & GCGACTCTGAGGAGGAACAAGA & CCAGCAGAAGGTGATCCAGACT \\
\hline$L I T 1$ & CGGTTATTGGTTATGCCAAAGG & CCAGAGTCCGCGATGAAAAT \\
\hline$B M G$ & ACACCAGCCAGGAAGGCCCA & AGCTTCGCCCAGCCGTAGGA \\
\hline Human $\alpha$-satellite DNA & TGACTTTGTCACAGCCCAAGATA & AATGCGGCATCTTCAAACCT \\
\hline Murine $\beta$-actin intronic DNA & ACCACTCTGTGTCCTTCGTTCG & ACTGCGCTCTCAAAAGGAGTGT \\
\hline & TTCAATTCCAACACTGTGCTGTCT & CTGTGGAGTGACTAAATGGAAACC \\
\hline
\end{tabular}

Table 4. Primer list for RT-qPCR 


\begin{tabular}{|l|l|l|}
\hline Name & Target Gene & Target Sequence $\mathbf{( 5}^{\prime}-\mathbf{3}^{\prime}$ ) \\
\hline Control & Renilla $[87]$ & CAAAGGAAACGGATGATAA \\
\hline Construct 1 & DLK1 $[88]$ & GGTGTCCATGAAAGAGCTC \\
\hline Construct 2 & DLK1 & GGTATCGTCTTCCTCAACAAG \\
\hline
\end{tabular}

Table 5. Plasmid shRNA sequences for producing stably transfected cell lines 


\section{CHAPTER 3: DLK1 EXPRESSION AND MEG3 PROMOTOR METHYLATION ARE NOVEL MARKERS OF AML PATIENT SURVIVAL}

\section{Introduction}

The overlap of primordial germ cell migration and transformation with the appearance of primitive hematopoiesis in the developing embryo suggests that these two phenomena are somehow linked. The developmental route of mouse PGCs begins with their specification in the epiblast at embryonic day 6.25 (E6.25), continues with their epigenetic reprogramming and migration through the extraembryonic endoderm, and ends with their deposition in the genital ridges by E12.5, where they finalize their epigenetic reprogramming and transformation into either male or female germ cells $[39,89]$. The appearance of PGCs at the allantois, a region of the extraembryonic yolk sac, at E7.5 occurs at a similar time and region as the first appearance of hemangioblasts and hematopoietic islands $[90,91]$. Similarly, PGCs enter the aorta-gonad-mesonsephros region of the embryo at E11, which is the region where HSCs first appear and where Oct3/4-expressing cells were recently isolated which could form hematopoietic colonies in vitro [42, 92]. Considering this, it is not surprising that GCTs have been associated with hematologic malignancies, or that mouse teratocarcinoma cells were able to differentiate into erythroid cells in vitro [56-58, 93].

For PGCs, hypomethylation of the IGF2-H19 ICR as a result of their epigenetic reprogramming serves not only as an artifact of the putative PGC origins of GCTs, but it is likely an important factor in the malignant transformation of these otherwise benign 
cells as well [18, 36-38]. Similarly, imprinted genes are key regulators of HSC quiescence and maintenance, and several studies have documented various genomic imprinting defects in certain blood disorders $[5,6,94]$. Among these disorders is the blood cancer AML, which is characterized by the malignant expansion of myeloid progenitor blast cells. Studies in HSC maintenance and aberrant genomic imprinting in AML have uncovered the potential roles of several imprinted genes, many of which are considered part of an imprinted gene network (IGN) deeply involved in the regulation of embryonic and somatic growth [94-96].

In an effort to determine whether IGN members may influence the outcomes of AML patients, I was involved in an initial screening of methylation at the DMRs of several loci which encode members of the IGN, including IGF2-H19, ZAC, PEG1, and PEG3. However, while there were methylation differences between AML patients and MNC control cells for the IGF2-H19, ZAC, and PEG1 DMRs, there were no associations between the methylation of these DMRs and either the complete remission or the survival of patients (Figures 6-8). Considering the importance of both the IGF2-H19 and DLK1-MEG3 loci for the generation of bimaternal mice and for the quiescence and maintenance of HSCs, the DMRs which control the expression of IGN members DLK1 and MEG3 became prospective targets for the screen. 

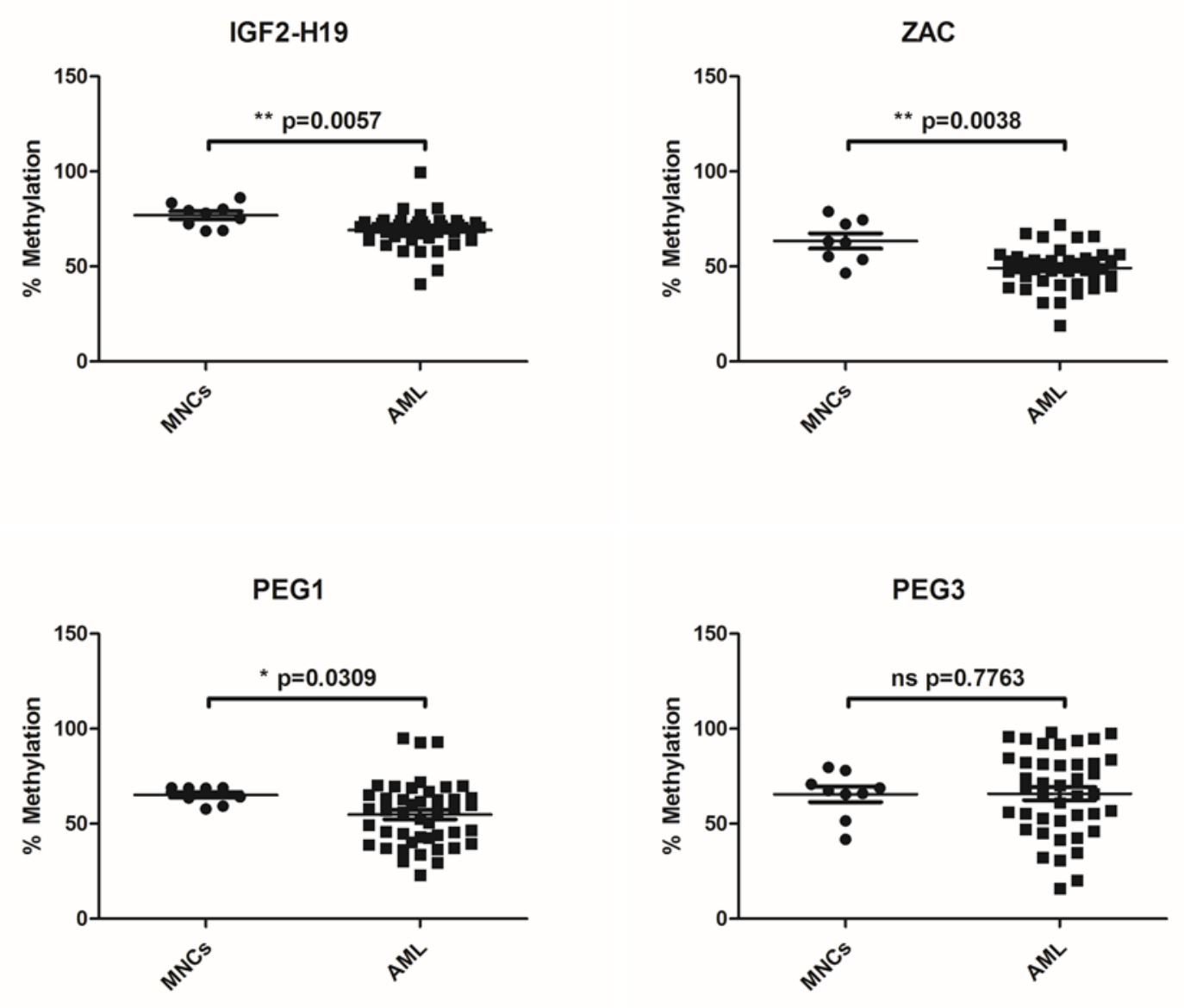

Figure 6. Leukemic cells exhibit differences in methylation at imprinted gene network loci compared to mononuclear cells

Comparison of COBRA results for normal human mononuclear cells (MNCs) and human mononuclear cells from AML patients (AML). Graphs represent the results of COBRA performed once per sample for each locus and analyzed using the Mann-Whitney U test. MNCs - IGF2-H19, PEG1, PEG3 n=9; ZAC n=8. AML - IGF2-H19, PEG1 n=45; PEG3, ZAC $\mathrm{n}=42 . \mathrm{ns}$ not significant, $* \mathrm{p}<0.05, * * \mathrm{p}<0.01$ 

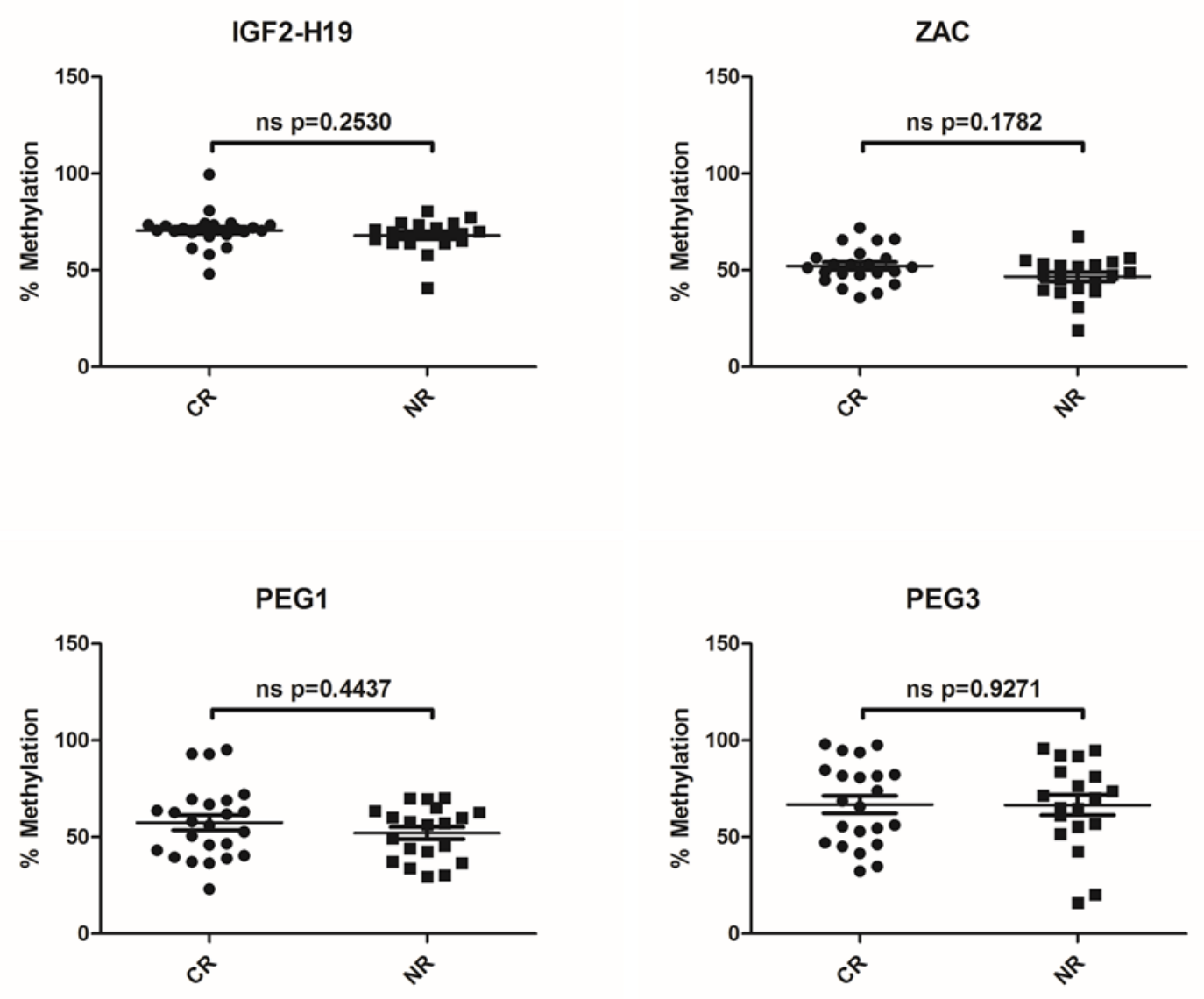

Figure 7. Methylation at imprinted gene network loci is not associated with complete remission in AML patients following the first round of induction therapy Comparison of COBRA results for human mononuclear cells from AML patients who responded $(\mathrm{CR})$ or did not respond $(\mathrm{NR})$ to treatment. Graphs represent the results of COBRA performed once per sample for each locus and analyzed using the Mann-Whitney U test. CR - IGF2-H19, PEG1 n=24; PEG3, ZAC n=22. NR - IGF2-H19, PEG1 n=20; PEG3, ZAC n=19. ns not significant 
IGF2-H19

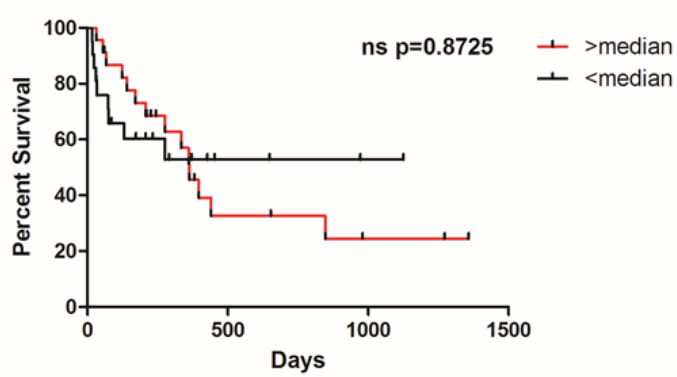

PEG1

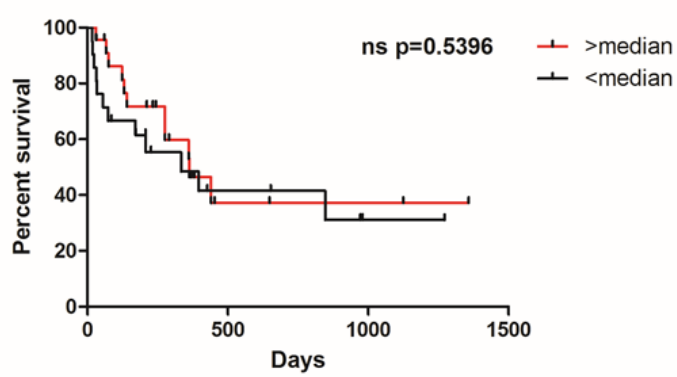

ZAC

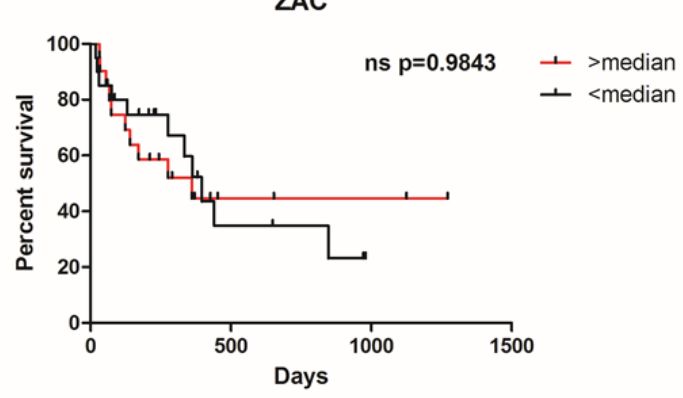

PEG3

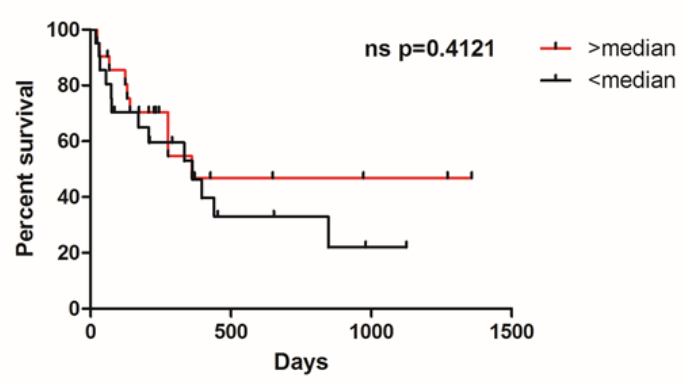

Figure 8. Methylation at imprinted gene network loci is not associated with AML patient survival

Comparison of COBRA results for human mononuclear cells from AML patients based on patient survival. Graphs represent the results of COBRA performed once per sample for each locus and analyzed based on the median methylation for each locus using the MantelCox test. >median methylation - IGF2-H19, PEG1 n=23; PEG3, ZAC n=21. <median methylation - IGF2-H19, PEG1, PEG3 n=21; ZAC n=20. ns not significant 
On the other hand, the DLK1-MEG3 locus encodes the growth-related proteincoding gene $D L K 1$ and the tumor-suppressing long noncoding RNA $M E G 3$, as well as the second largest cluster of miRNAs in the human genome. While initially hypothesized to be under an imprinting control scheme similar to that of the $I G F 2-H 19$ locus, the presence of multiple DMRs and CTCF binding sites serve to illustrate a much less simple mechanism [97, 98]. Nonetheless, the intergenic control DMR (IG-DMR) sits atop the DMR hierarchy for this locus and thus exerts control over the expression of $D L K 1, M E G 3$, and the downstream miRNAs [99]. Previous studies in AML and acute promyelocytic leukemia (APL) revealed methylation defects in the DLK1-MEG3 locus which affected the expression of $D L K 1$ and several of the downstream miRNAs, and hypermethylation of the MEG3 promotor was recently associated with decreased survival of AML patients [60, 98, 100]. However, multiple $\mathrm{CpG}$ sites exist within the $M E G 3$ promotor region, and the degree of methylation in this region was not used for associations with survival. Similarly, the expression of $D L K 1$ and $M E G 3$ have not been previously investigated for their roles in AML patient survival. Therefore, I hypothesized that methylation at select CpG sites throughout the DLK1-MEG3 locus (Figure 9), along with the expression of DLK1 and MEG3, could be associated with AML patient outcomes. 


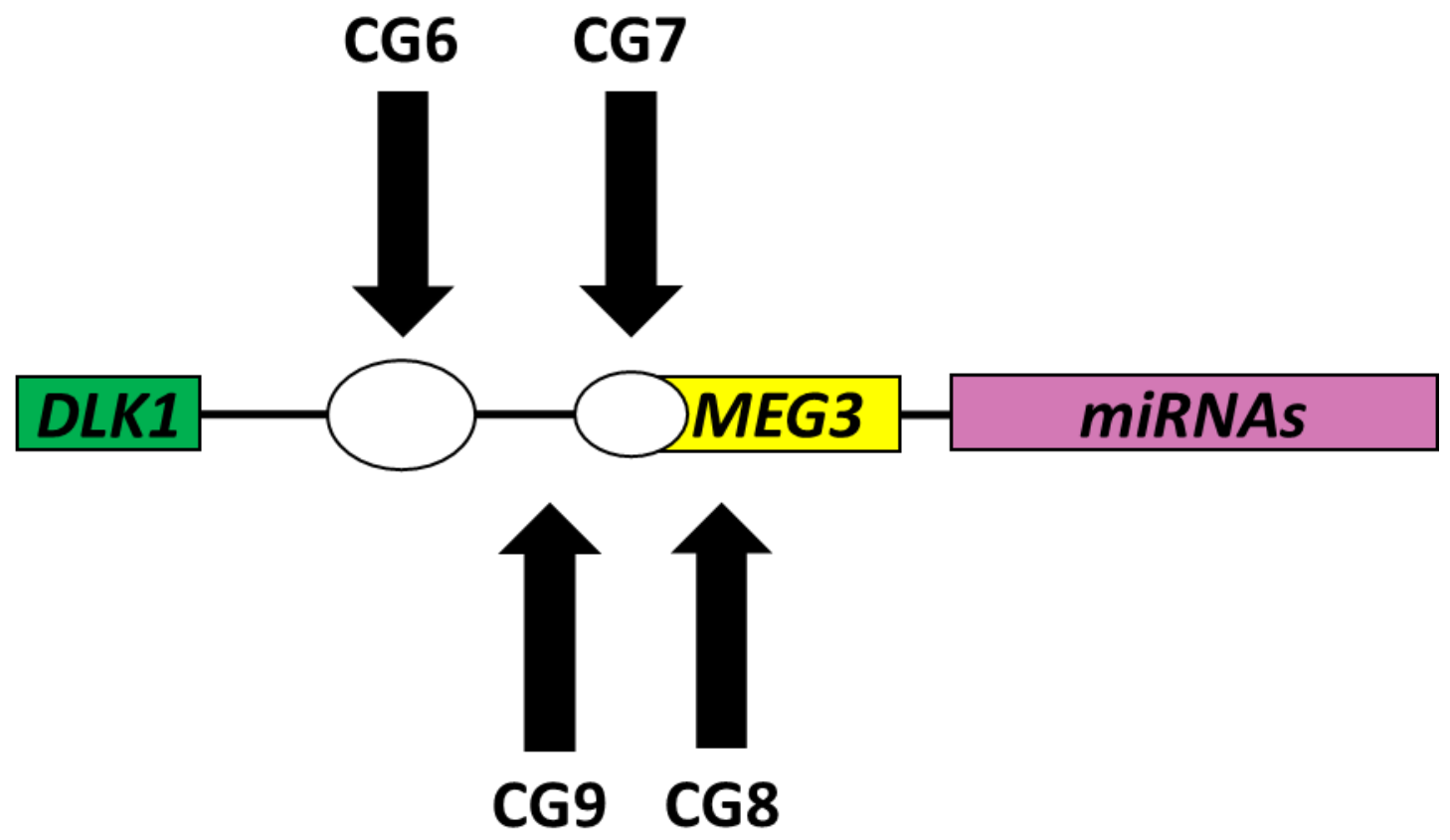

Figure 9. CpG sites within the DLK1-MEG3 locus

Several imprinted (top arrows) and non-imprinted (bottom arrows) $\mathrm{CpG}$ sites exist within the DLK1-MEG3 locus. White circles represent the IG-DMR (left) and MEG3 DMR (right). 


\section{Results}

\section{Methylation at select CpG sites within the DLK1-MEG3 locus is associated with AML}

patient outcomes

Previous investigations into the DLK1-MEG3 locus in blood disorders have revealed significant associations between the presence of dysplastic cells and aberrant methylation at select sites which contain many CpGs [60, 98, 100, 101]. Specifically, hypermethylation at the MEG3 promotor has been observed in MDS, multiple myeloma, and AML patient samples [100, 101]. Because hypermethylation of the $M E G 3$ promotor was recently associated with decreased AML patient survival, I investigated the methylation of several CpG sites within the DLK1-MEG3 locus to determine whether this association involves the disruption of genomic imprinting [100].

COBRA results from control MNCs and AML samples revealed significant differences for CG6, CG7, and CG8, but not for CG9 (Figure 10). A previous report utilizing a more detailed sequencing method found no overall differences between AML patients and control samples for the same CG6 region [60]. However, their results for AML patients show hypermethylation of the same CpGs which are targeted by BstUI in my assay, in agreement with my results. Similarly, this report also analyzed amplicons which lie in close proximities to the CG7, CG8, and CG9 amplicons, and found no differences in methylation between AML and control samples. However, none of their amplicons directly overlaps with CG7, CG8, or CG9, and in addition to the fact that their AML samples often showed considerable variation in methylation, it is also worth noting that their analyses were for a subset of $D L K 1$-overexpressing patients. 
Analyses of the response of patients to the first round of induction therapy based on methylation at each $\mathrm{CpG}$ site uncovered a slight, though significant, increase in the methylation of CG8 for patients who went into complete remission (CR) compared to nonresponders (NR) (Figure 11). This is surprising, as there was no statistical difference in the methylation at CG8 between AML and control MNCs. More importantly, however, survival curves generated based on the median methylation of AML samples for each CpG sites show significant associations between increased survival and higher methylation at CG7 and CG9 (Figure 12). Because CG7 and CG9 lie within the MEG3 promotor region, these results seemingly contradict a previous report which associated higher methylation of the MEG3 promotor with decreased survival in AML [100]. This is likely due to several possible factors centered around their method of methylation analysis for this region. The authors used methylation-specific PCR, in which one set of primers amplified a methylated allele and the other set of primers amplified an unmethylated allele, to determine whether there was aberrant methylation within the region. In addition to being arguably less sensitive for analyzing methylation than COBRA, the authors used sets of primers which did not overlap and instead were separated by over $1.3 \mathrm{~kb}$. In fact, their methylated allele primer set amplified a region of DNA $150 \mathrm{bp}$ downstream of CG9 (not imprinted), and their unmethylated allele primer set amplified a region of DNA within CG7 (imprinted), which could have produced inconsistent results due to the unique methylation patterns of genomic imprints. Thus, my results using COBRA may offer novel insight into the role of MEG3 promotor methylation in the survival outcome of AML patients.

Further investigation into the associations between $\mathrm{CpG}$ site methylation and patient outcome yielded interesting results. A Spearman's Rho test did not show a 
correlation between age and methylation at CG6 (rho=-0.16, p=0.28), CG8 (rho-=0.16, $\mathrm{p}=0.28)$, or CG9 $(\mathrm{rho}=-0.13, \mathrm{p}=0.39)$, but did show a correlation between CG7 methylation and patient age $(\mathrm{rho}=-0.39, \mathrm{p}=0.01)$. Because the type of treatment offered for AML before and after 60 years of age are different, I checked the clinical response of patients using this age as a cutoff (Figure 13). No statistically significant differences in methylation were found between patients based on their response for either age group. Due to the limited number of patients over 60, a similar investigation into the association between methylation and survival based on age was carried out only for patients under 60, which yielded a strong association between high methylation and increased survival.

Gene expression at the DLK1-MEG3 locus is regulated by both genomic imprinting and promotor methylation, offering the possibility that $\mathrm{CpG}$ sites within this locus may regulate gene expression independently as well as cooperatively. Similarly, genes expressed from this locus serve opposing roles in cell proliferation, further complicating the overall influence of aberrant methylation at this locus on malignancy. Therefore, I analyzed the associations between methylation at multiple sites and patient survival (Figure 14; $\mathrm{p}$ values listed in Table 6). I found significant differences in survival between patients who had low methylation at all sites compared to patients who had high methylation at 1 , 2, or 3 sites total throughout this locus, with no differences between any other groups. Of these CpG sites, 2 are imprinted, 2 are not imprinted, and 2 are within the promotor region of $M E G 3$. Analyses of the associations between survival and low methylation at all sites, up to 2 sites of high methylation, and over 2 sites of high methylation illustrated an intriguing stepwise pattern of increasing survival with increasing methylation, though the difference between 1-2 sites and 3-4 sites was not statistically significant. Similarly, a 
stepwise pattern was found when analyzing only those $\mathrm{CpG}$ sites found within the $M E G 3$ promotor, albeit in an unexpected manner, where the difference between 1 site and 2 sites was also not statistically significant. 

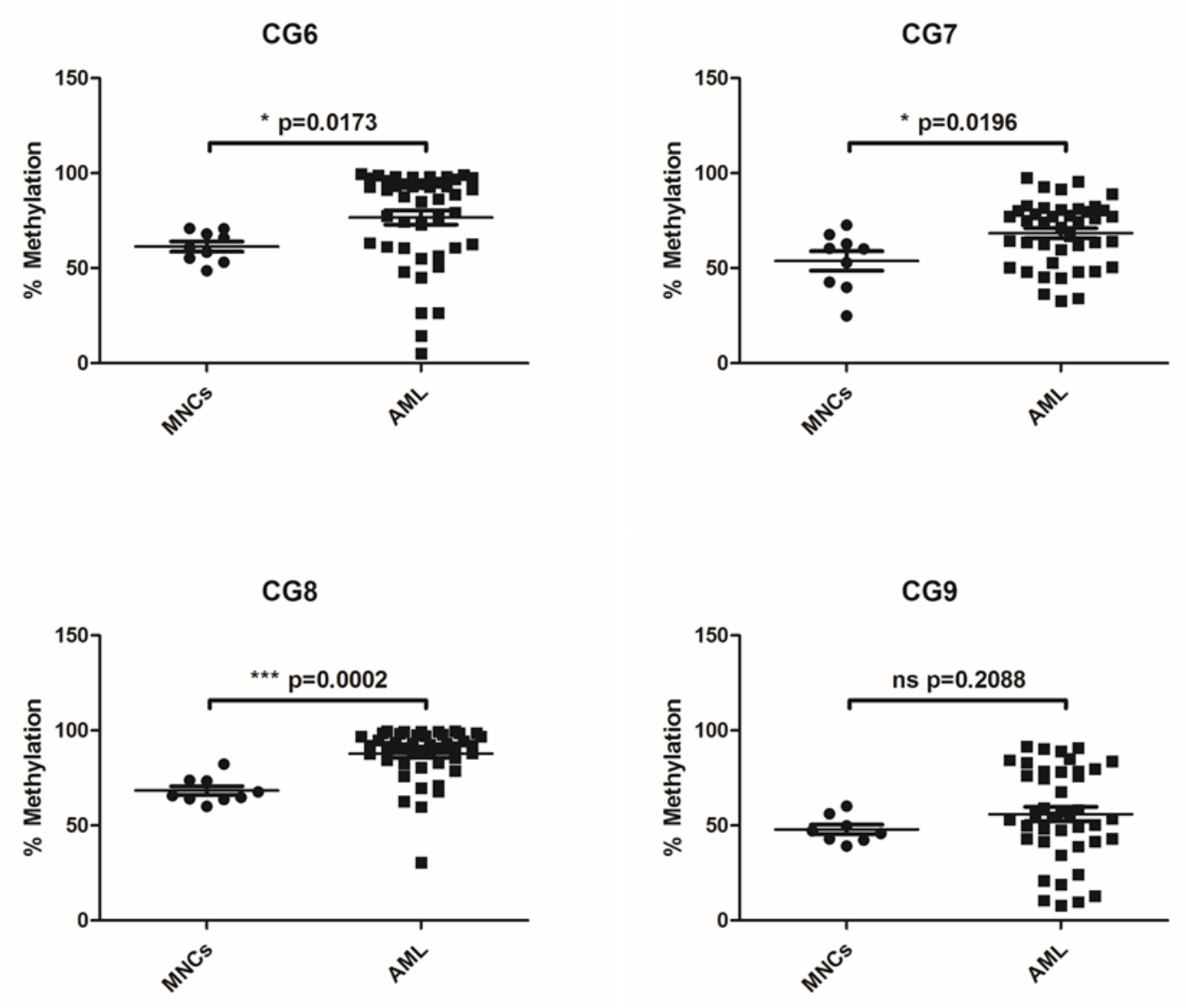

Figure 10. Leukemic cells exhibit differences in methylation at CG6, CG7, and CG8 within the DLK1-MEG3 locus compared to mononuclear cells Comparison of COBRA results for normal human mononuclear cells (MNCs) and human mononuclear cells from AML patients (AML). Graphs represent the results of COBRA performed once per sample for each $\mathrm{CpG}$ site and analyzed using the Mann-Whitney $\mathrm{U}$ test. MNCs - CG6, CG7, CG8 n=9; CG9 n=8. AML - CG6, CG8 n=44; CG7, CG9 n=42. ns not significant, $* \mathrm{p}<0.05, * * * \mathrm{p}<0.001$ 

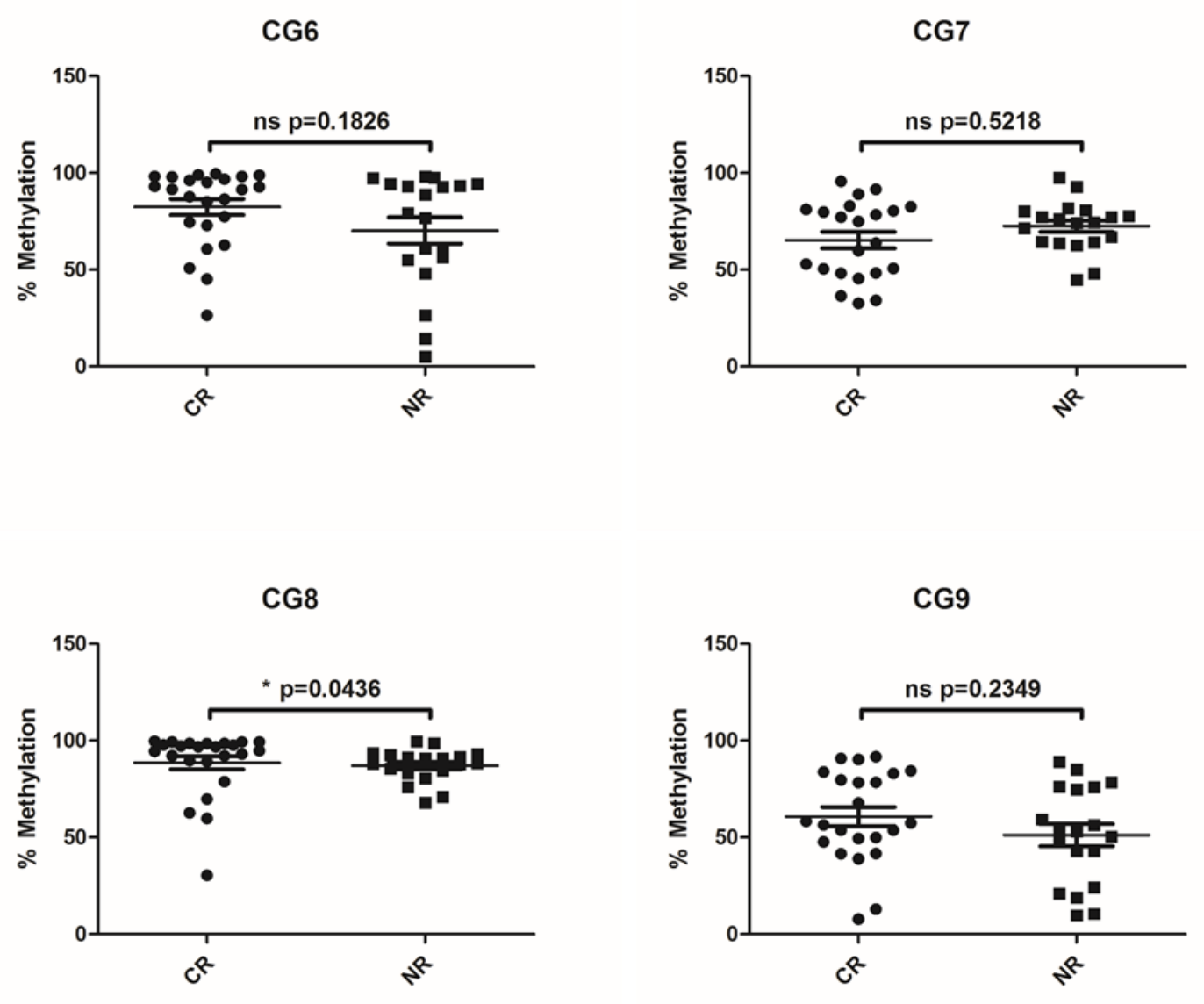

Figure 11. Methylation at individual CpG sites within the DLK1-MEG3 locus is not associated with complete remission in AML patients following the first round of induction therapy

Comparison of COBRA results for human mononuclear cells from AML patients who responded $(\mathrm{CR})$ or did not respond $(\mathrm{NR})$ to treatment. Graphs represent the results of COBRA performed once per sample for each $\mathrm{CpG}$ site and analyzed using the MannWhitney U test. CR - CG6, CG8 n=24; CG7 n=22; CG9 n=23. NR - CG6, CG7, CG8, CG9 $n=19$. ns not significant, $* \mathrm{p}<0.05$ 

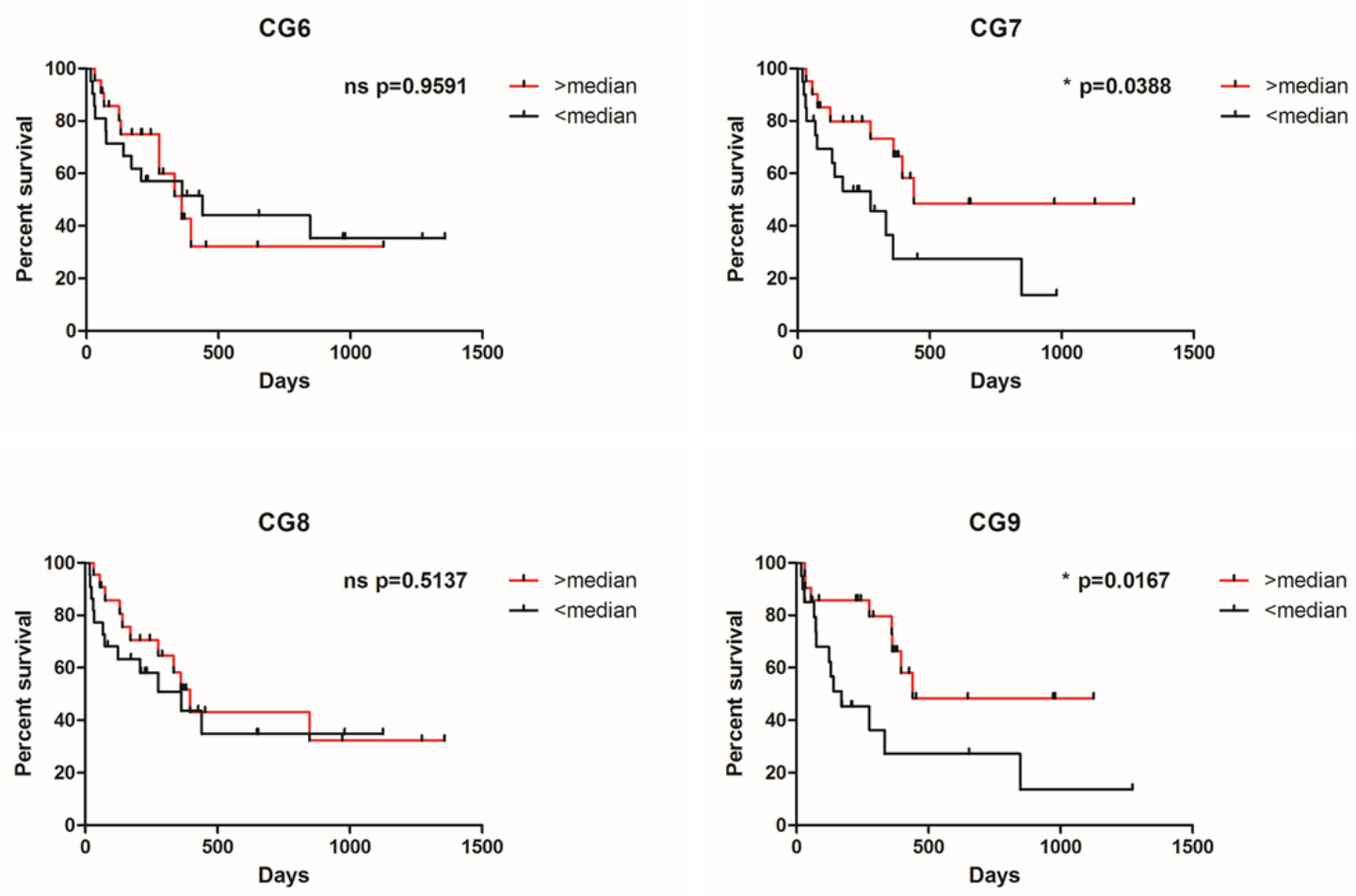

Figure 12. Methylation at CG7 and CG9 within the promotor region of MEG3 is associated with AML patient survival

Comparison of COBRA results for human mononuclear cells from AML patients based on patient survival. Graphs represent the results of COBRA performed once per sample for each CpG site and analyzed based on the median methylation for each locus using the Mantel-Cox test. >median methylation - CG6, CG8 n=22; CG7, CG9 n=21. <median methylation - CG6 n=21; CG7, CG9 n=20; CG8 n=22. ns not significant, * $\mathrm{p}<0.05$ 
A.
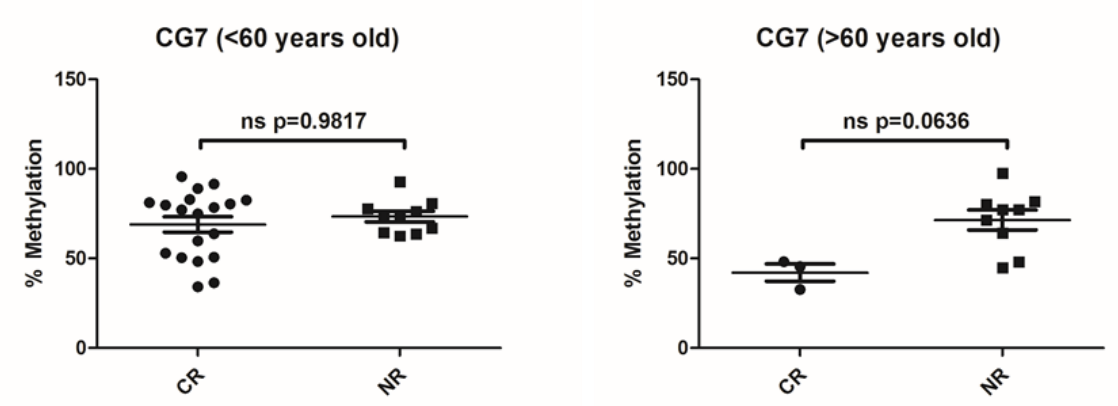

B.

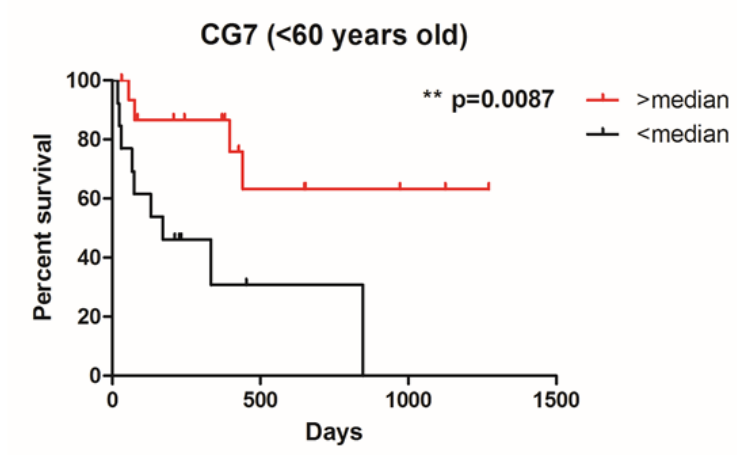

Figure 13. Methylation at CG7 in the promotor region of $M E G 3$ does not exhibit agerelated associations with complete remission in AML patients following the first round of induction therapy

(A) Comparison of COBRA results for human mononuclear cells from AML patients of different age groups who responded (CR) or did not respond (NR) to treatment. Graphs represent the results of COBRA performed once per sample for CG7 and analyzed using the Mann-Whitney U test. CR $<60$ years $n=19$. NR $<60$ years $n=10 . C R>60$ years $n=3$. NR $>60$ years $n=9$. (B) Comparison of COBRA results for human mononuclear cells from AML patients based on patient survival. Graphs represent the results of COBRA performed once per sample for CG7 and analyzed based on the median methylation for CG7 of patients $<60$ years using the Mantel-Cox test. >median methylation $n=16$. <median methylation $\mathrm{n}=13$. ns not significant, $* \mathrm{p}<0.05$, ** $\mathrm{p}<0.01$ 

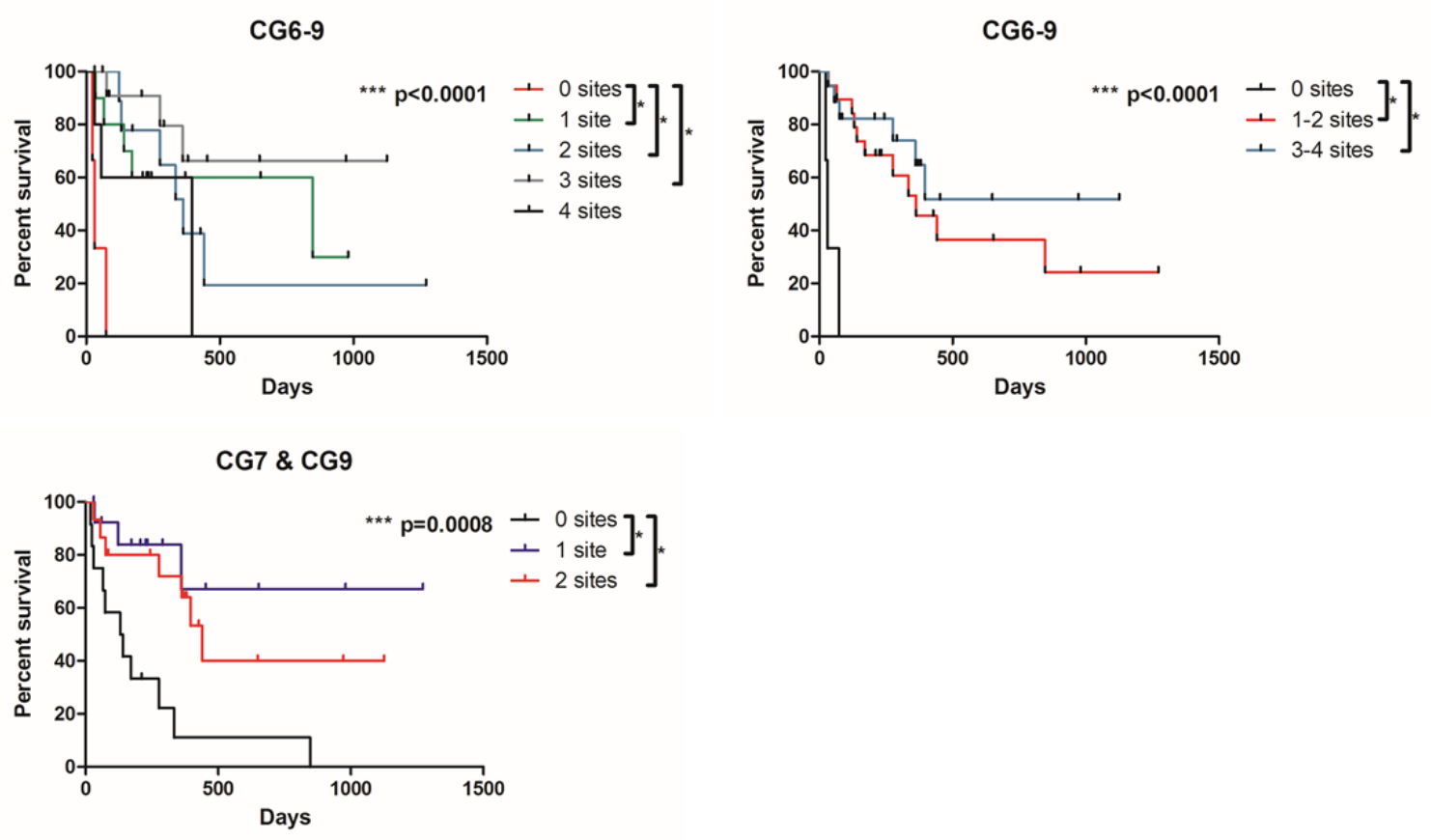

Figure 14. Combining methylation results from multiple DLK1-MEG3 CpG sites uncovers unique associations between AML patient survival and methylation at this locus

Comparison of COBRA results for human mononuclear cells from AML patients based on patient survival. Graphs represent the results of COBRA performed once per sample for each $\mathrm{CpG}$ site and analyzed based on the median methylation for each $\mathrm{CpG}$ site using the Mantel-Cox test, where "0 sites" denotes patients for whom the methylation at each CpG site was lower than the median methylation for that site, " 1 site" denotes patients for whom the methylation at one $\mathrm{CpG}$ site was higher than the median methylation for that site, etc. CG6-9 -0 sites $n=3$ (both graphs); 1 site $n=10 ; 2$ sites $n=9 ; 3$ sites $n=13 ; 4$ sites $n=5,1-2$ sites $\mathrm{n}=19 ; 3-4$ sites $\mathrm{n}=18 . \mathrm{CG} 7,9-0$ sites $\mathrm{n}=12 ; 1$ site $\mathrm{n}=14 ; 2$ sites $\mathrm{n}=15 . * * * \mathrm{p}<0.001$ for Mantel-Cox test; * $\mathrm{p}<0.005$ for Bonferroni (CG6-9 left graph); * $\mathrm{p}<0.0167$ (CG6-9 right graph, CG7 \& CG9 graph) 


\begin{tabular}{|l|l|l|r|c|}
\hline CG6-9 $\mathbf{( 0 , 1 , 2 , 3 , 4 )}$ & 1 site & 2 sites & 3 sites & 4 sites \\
\hline 0 sites & 0.0027 & 0.0002 & $<0.0001$ & 0.0733 \\
1 site & & 0.7903 & 0.2290 & 0.4016 \\
2 sites & & & 0.1546 & 0.5870 \\
3 sites & & & & 0.1190 \\
\hline
\end{tabular}

\begin{tabular}{|l|c|c|}
\hline CG6-9 (0, 1-2, 3- & $1-2$ & $\begin{array}{c}\text { 3-4 } \\
\text { 4) }\end{array}$ \\
\hline 0 sites & $<0.0001$ & \multicolumn{1}{c|}{ sites } \\
\hline $1-2$ sites & & 0.0001 \\
& & 0.3434 \\
\hline
\end{tabular}

\begin{tabular}{|l|l|c|}
\hline CG7 \& 9 $\mathbf{( 0 , 1 , 2 )}$ & 1 site & 2 sites \\
\hline 0 sites & 0.0012 & 0.0051 \\
1 sites & & 0.3941 \\
\hline
\end{tabular}

Table 6. Individual p values for Figure 14 


\section{DLK1 expression is associated with AML patient outcomes, but not with CpG site methylation}

Several studies have recently emerged which analyzed the expression of imprinted genes within the DLK1-MEG3 locus in AML patient samples [60, 100, 102, 103]. Whereas the expression of MEG3 was found to be downregulated in both AML patient samples and cell lines, conflicting data has appeared for the expression of DLK1 in AML patient samples, and investigations into the outcome of patients based on the expression of these genes are lacking $[60,63]$. My results show that the expression of both $D L K 1$ and $M E G 3$ is significantly decreased in AML patients compared to control MNCs (Figure 15). However, though no studies have directly investigated the DLK1/MEG3 expression ratio in AML patient samples, these results for $D L K 1$ and $M E G 3$ surprisingly did not amount to any difference in the DLK1/MEG3 expression ratio between AML patients and control MNCs. Some AML samples exhibited high expression of these genes, and AML samples appeared to group together based on their DLK1/MEG3 expression ratio.

Considering my results from comparing $\mathrm{CpG}$ site methylation with patient outcomes, these variations in $D L K 1$ and $M E G 3$ expression among AML samples suggested that similar associations between expression patterns and patient outcomes may exist as well. While no associations between complete remission and the expression of $D L K 1$, $M E G 3$, or the DLK1/MEG3 expression ratio were found (Figure 16), high expression of DLK1 was associated with decreased patient survival (Figure 17). This is an interesting observation, as DLK1 is important for maintaining stem-like hematopoietic cells and may contribute to their accumulation, and the expression of $D L K 1$ was previously found to increase during the progression of $\operatorname{MDS}[61,63,104]$. 
The lack of association between $M E G 3$ expression and patient outcomes was unexpected, as CG7 and CG9 both lie within the MEG3 promotor and were associated with patient survival. Decreased expression of $M E G 3$ was also recently implicated in the development of AML [62]. Investigating potential associations between methylation of $\mathrm{CpG}$ sites within the DLK1-MEG3 locus and the expression of DLK1, MEG3, and the DLK1/MEG3 expression ratio in AML patients did not reveal any such associations for individual (Figures 18-21) or combined CpG sites (Figures 22, 23). While this was unexpected, the variability in the expression of $D L K 1$ and $M E G 3$ and of the $D L K 1 / M E G 3$ expression ratio among AML patients, combined with a previous report which described biallelic expression of $D L K 1$ in AML patients, suggests that biallelic expression of these genes is likely undermining the search for potential associations between their expression and $\mathrm{CpG}$ site methylation [60]. This report also found that control over mono- and biallelic expression of $D L K 1$ was regulated by a CpG site within the $D L K 1$ promotor, but not by $\mathrm{CpG}$ sites located in close proximities to each of the $\mathrm{CpG}$ sites I tested. Thus, investigating the biallelic expression of $D L K 1$ and $M E G 3$ in these patients in the future could be valuable for re-analysis of these potential associations. 

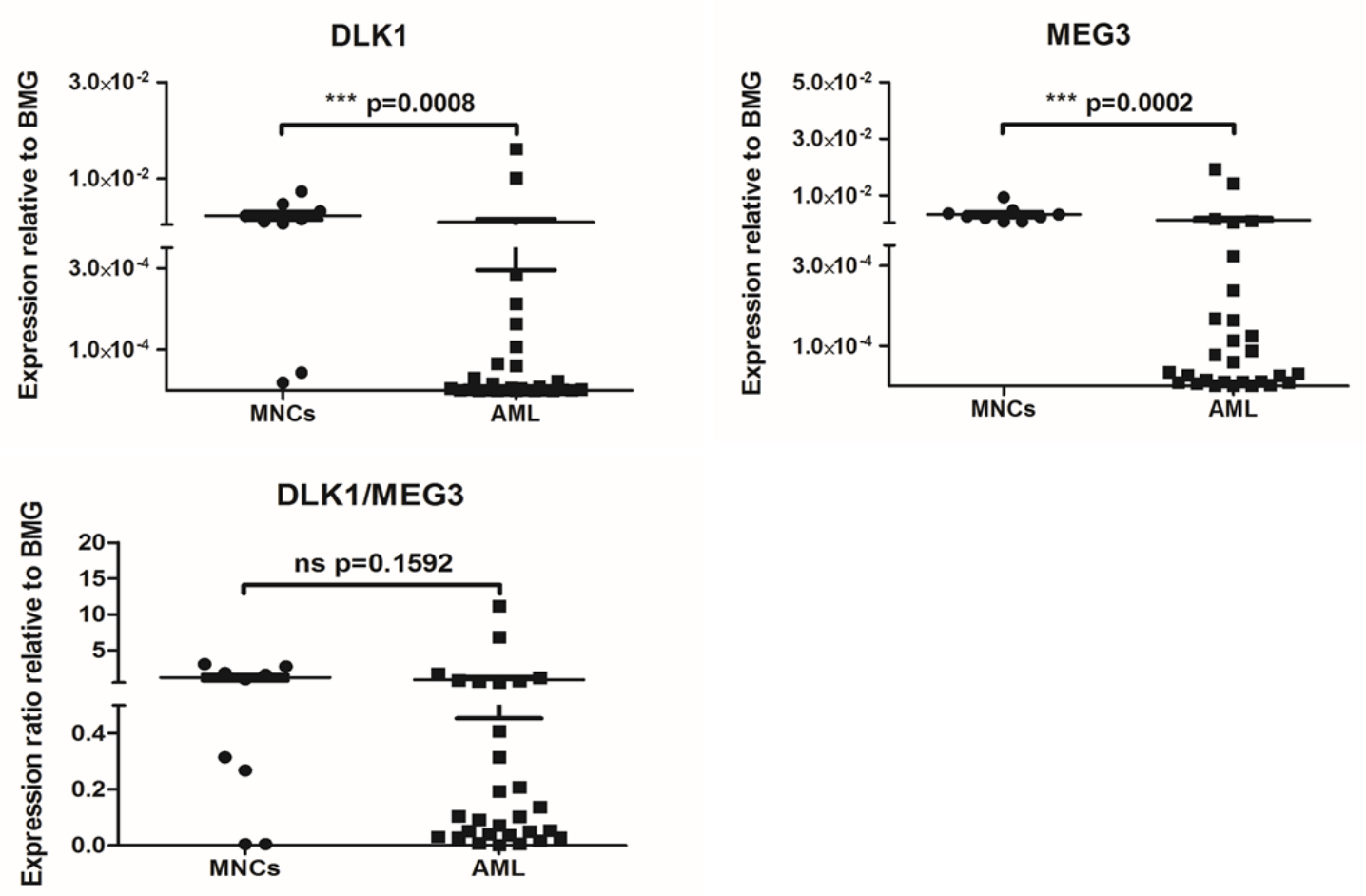

Figure 15. Leukemic cells exhibit lower DLK1 and MEG3 expression than mononuclear cells

Comparison of RT-qPCR results for normal human mononuclear cells (MNCs) and human mononuclear cells from AML patients (AML). Graphs represent the results of RT-qPCR performed once per sample in duplicate for each gene, where the average for each sample was used for analysis with the Mann-Whitney U test. MNCs - DLK1, MEG3, DLK1/MEG3 n=9. AML $-D L K 1, M E G 3, D L K 1 / M E G 3 \mathrm{n}=29$. ns not significant, $* * * \mathrm{p}<0.001$ 
DLK1
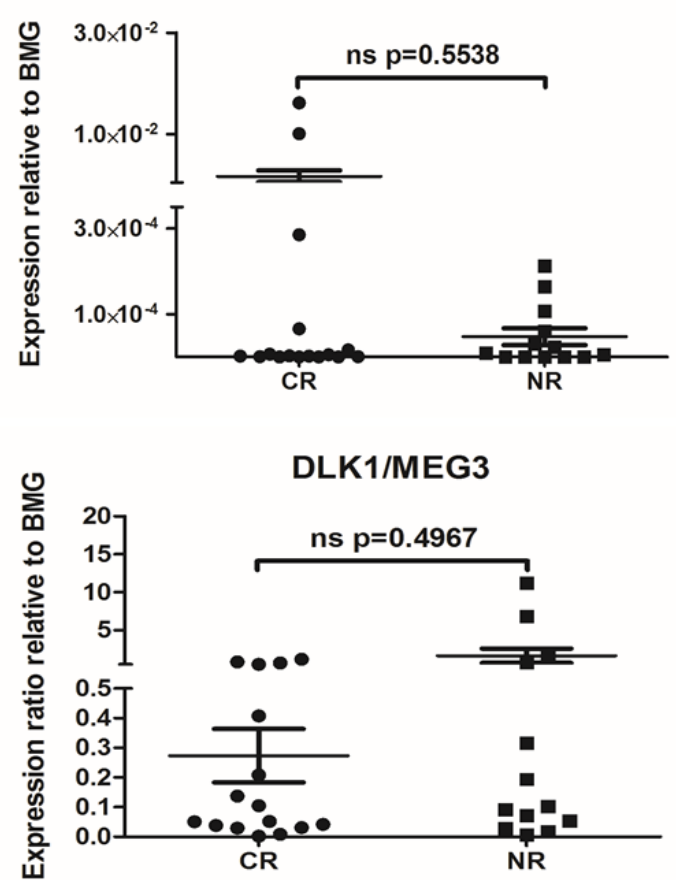

MEG3

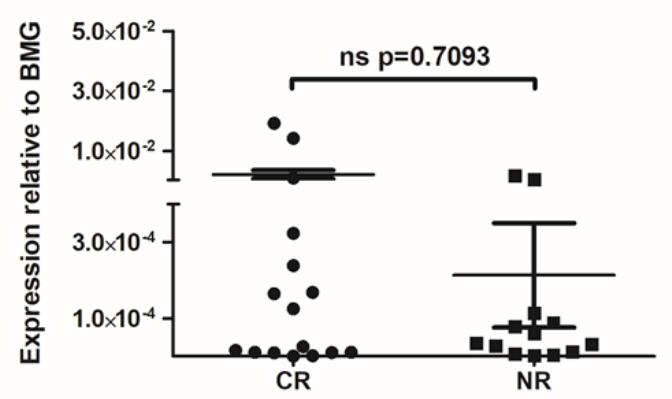

Figure 16. DLK1 and MEG3 expression is not associated with complete remission in AML patients following the first round of induction therapy

Comparison of RT-qPCR results for human mononuclear cells from AML patients who responded $(\mathrm{CR})$ or did not respond (NR) to treatment. Graphs represent the results of RTqPCR performed once per sample in duplicate for each gene, where the average for each sample was used for analysis with the Mann-Whitney U test. CR - DLK1, MEG3, DLK1/MEG3 n=16. NR - DLK1, MEG3, DLK1/MEG3 n=13. ns not significant 
DLK1

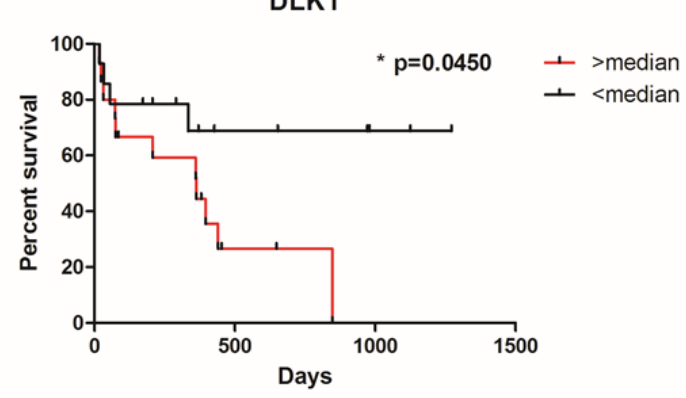

DLK1/MEG3

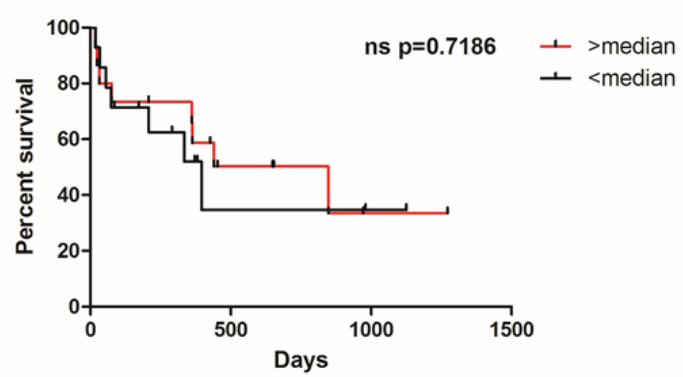

MEG3

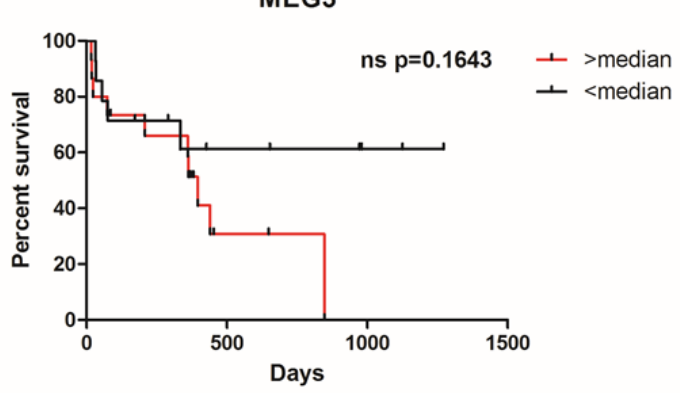

Figure 17. DLK1 expression is associated with AML patient survival

Comparison of RT-qPCR results for human mononuclear cells from AML patients based on patient survival. Graphs represent the results of RT-qPCR performed once per sample in duplicate for each gene, where the average for each sample was used for analysis with the Mantel-Cox test based on the median expression for each gene. >median expression DLK1, MEG3, DLK1/MEG3 n=14. <median expression - DLK1, MEG3, DLK1/MEG3 $\mathrm{n}=15 . \mathrm{ns}$ not significant, $* \mathrm{p}<0.05$ 

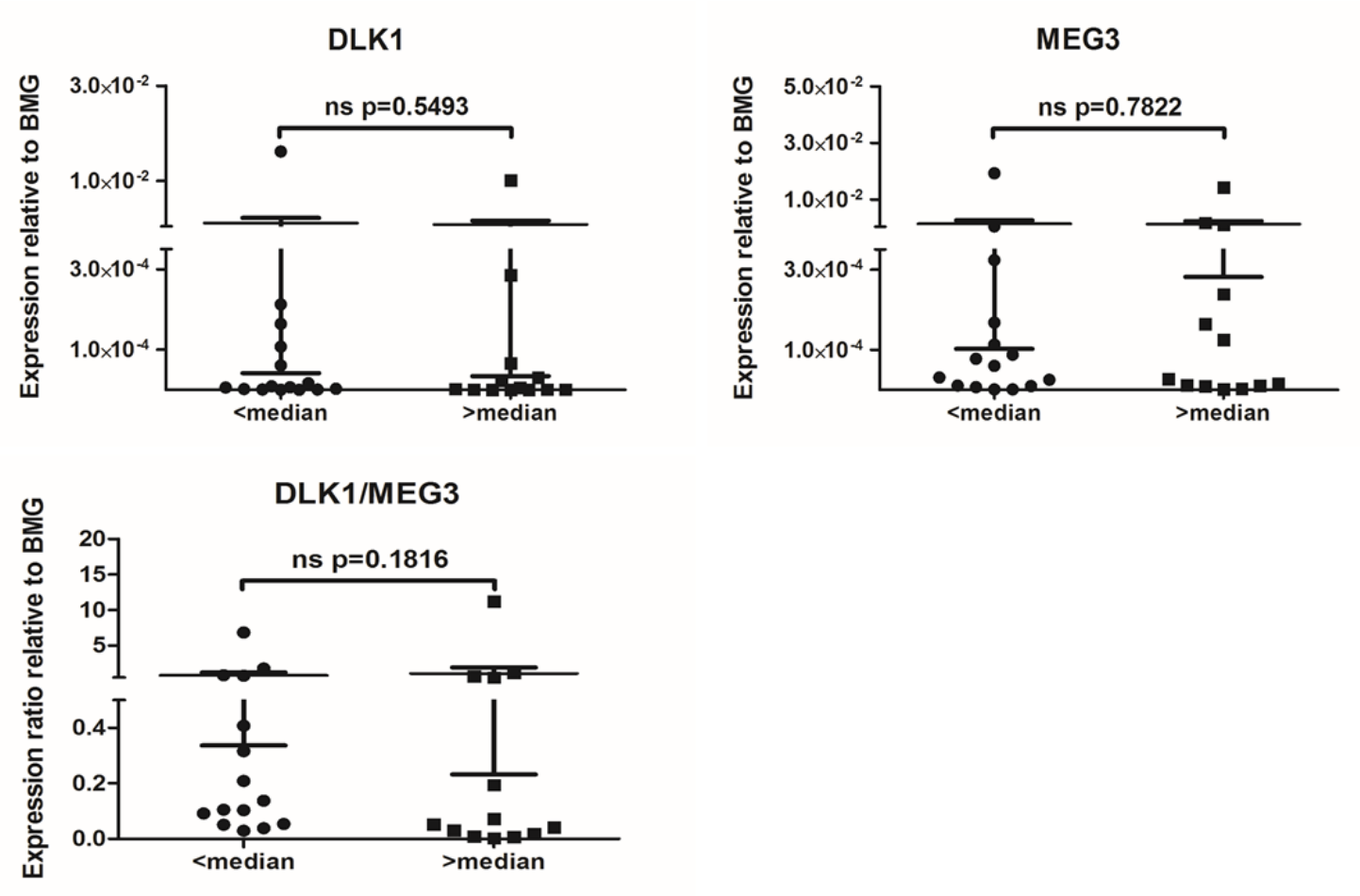

Figure 18. DLK1 and MEG3 expression is not associated with methylation at CG6

Comparison of RT-qPCR results for human mononuclear cells from AML patients based on methylation at CG6. Graphs represent the results of RT-qPCR performed once per sample in duplicate for each gene, where the average for each sample was used for analysis with the Mann-Whitney U test based on the median methylation at CG6 for that sample. >median methylation - DLK1 n=14; MEG3, DLK1/MEG3 n=13. <median methylation DLK1,MEG3, DLK1/MEG3 n=15. ns not significant 

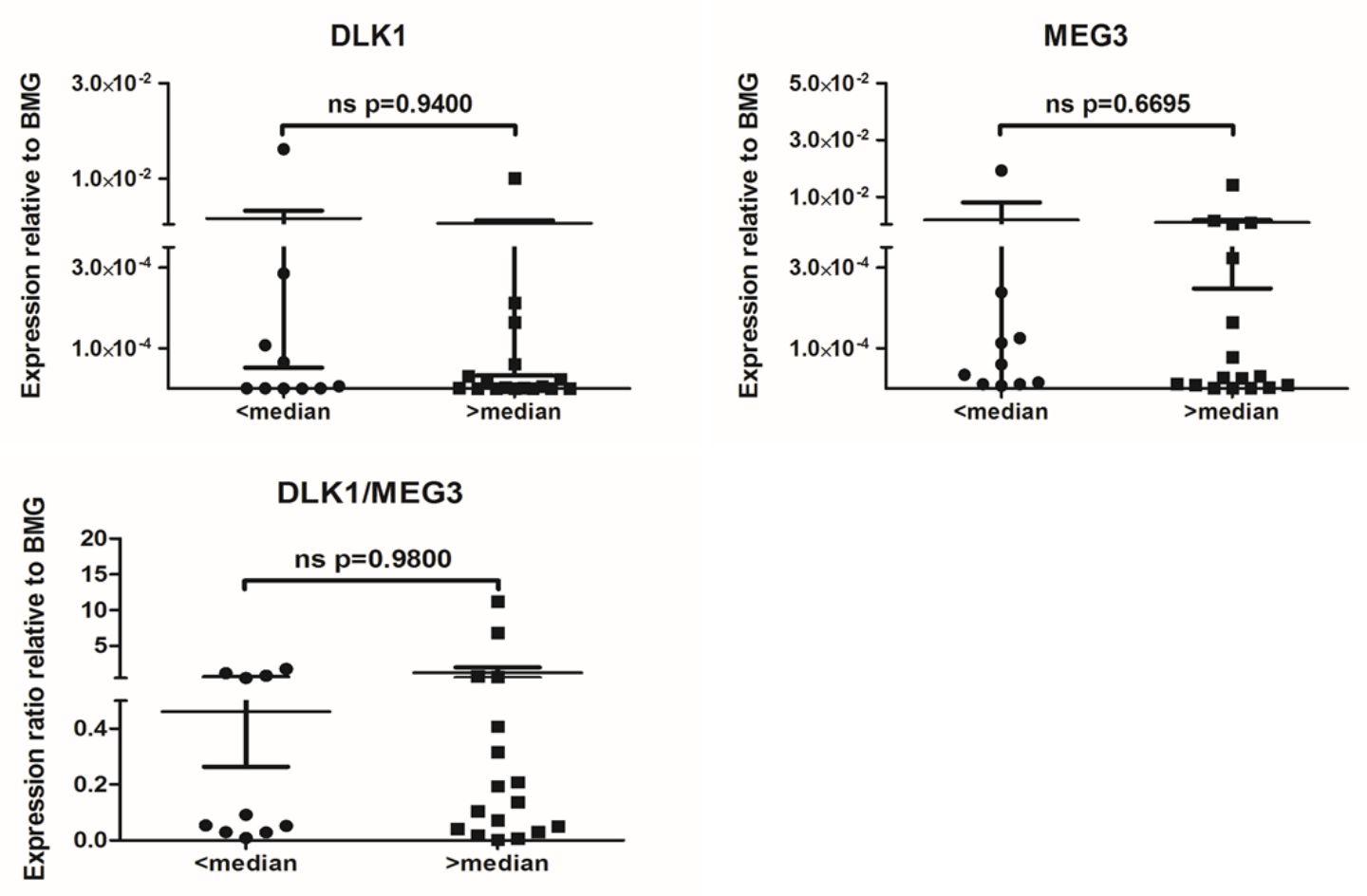

Figure 19. DLK1 and MEG3 expression is not associated with methylation at CG7

Comparison of RT-qPCR results for human mononuclear cells from AML patients based on methylation at CG7. Graphs represent the results of RT-qPCR performed once per sample in duplicate for each gene, where the average for each sample was used for analysis with the Mann-Whitney U test based on the median methylation at CG7 for that sample. >median methylation $-D L K 1, M E G 3, D L K 1 / M E G 3 \mathrm{n}=17$. <median methylation $-D L K 1$, MEG3, DLK1/MEG3 n=10. ns not significant 

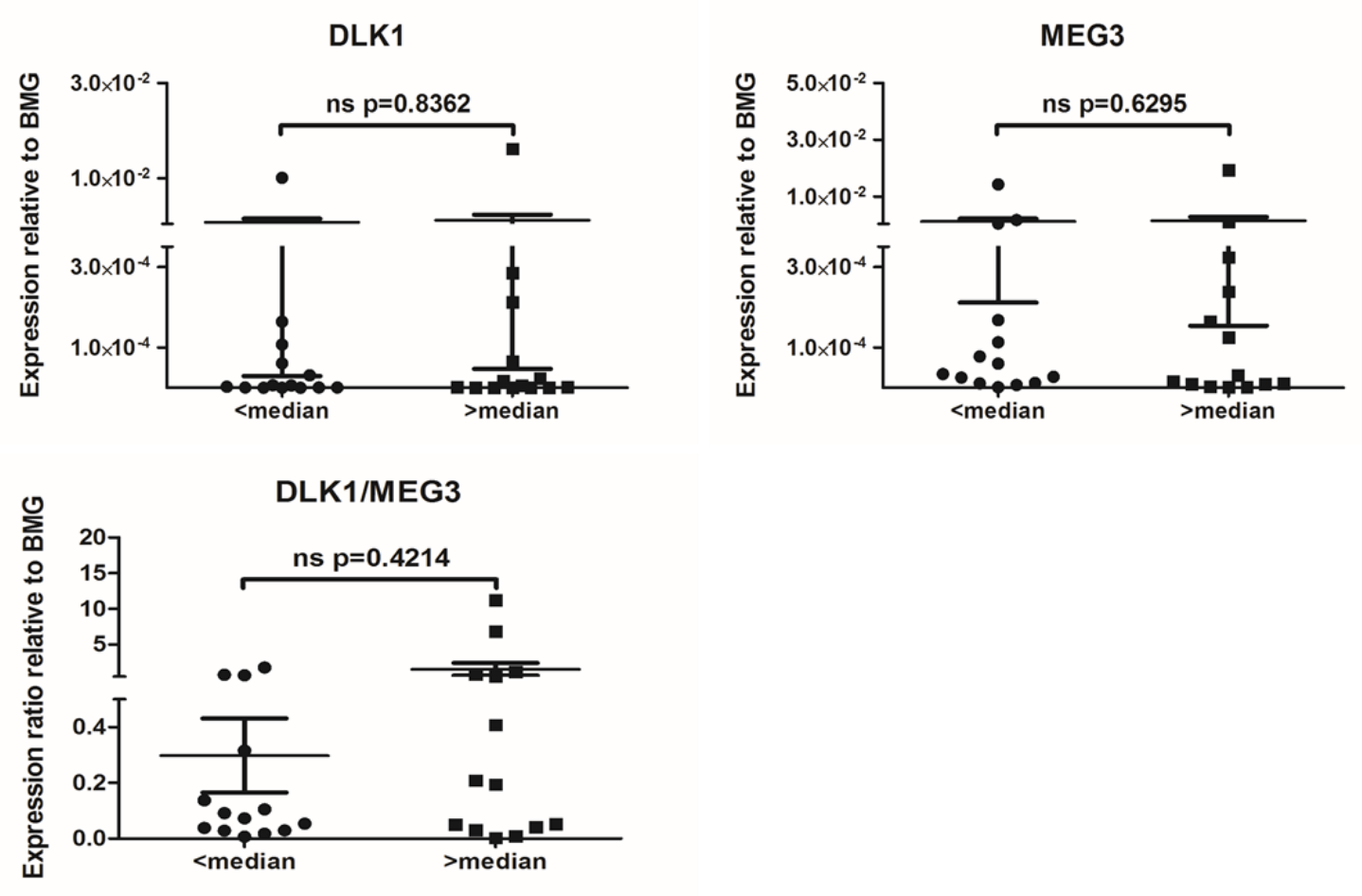

Figure 20. DLK1 and MEG3 expression is not associated with methylation at CG8

Comparison of RT-qPCR results for human mononuclear cells from AML patients based on methylation at CG8. Graphs represent the results of RT-qPCR performed once per sample in duplicate for each gene, where the average for each sample was used for analysis with the Mann-Whitney U test based on the median methylation at CG8 for that sample. >median methylation $-D L K 1, M E G 3, D L K 1 / M E G 3 \mathrm{n}=14$. <median methylation $-D L K 1$, MEG3, DLK1/MEG3 n=14. ns not significant 

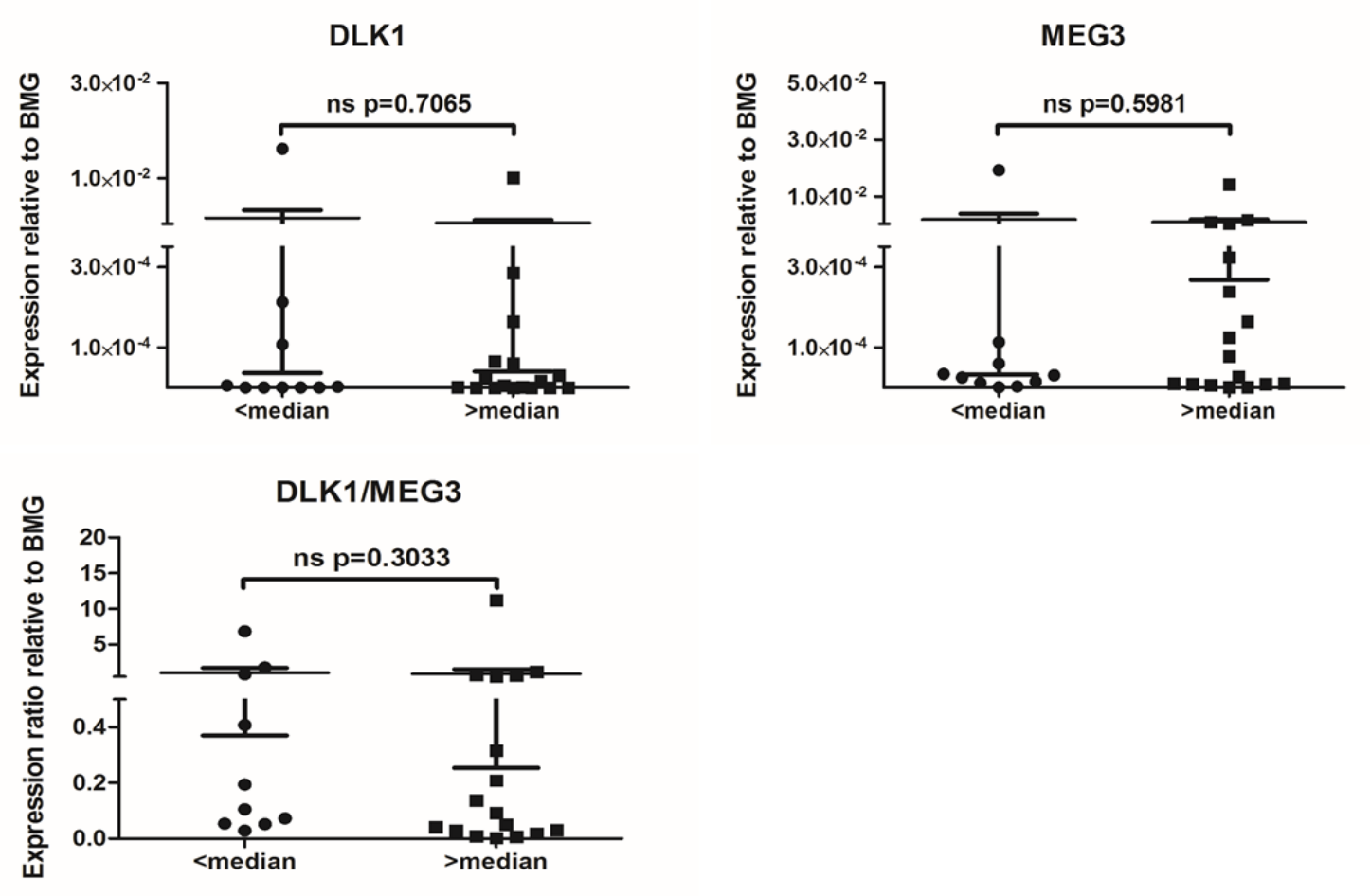

Figure 21. DLK1 and MEG3 expression is not associated with methylation at CG9

Comparison of RT-qPCR results for human mononuclear cells from AML patients based on methylation at CG9. Graphs represent the results of RT-qPCR performed once per sample in duplicate for each gene, where the average for each sample was used for analysis with the Mann-Whitney U test based on the median methylation at CG9 for that sample. >median methylation $-D L K 1, M E G 3, D L K 1 / M E G 3 \mathrm{n}=17$. <median methylation $-D L K 1$, MEG3, DLK1/MEG3 n=10. ns not significant 

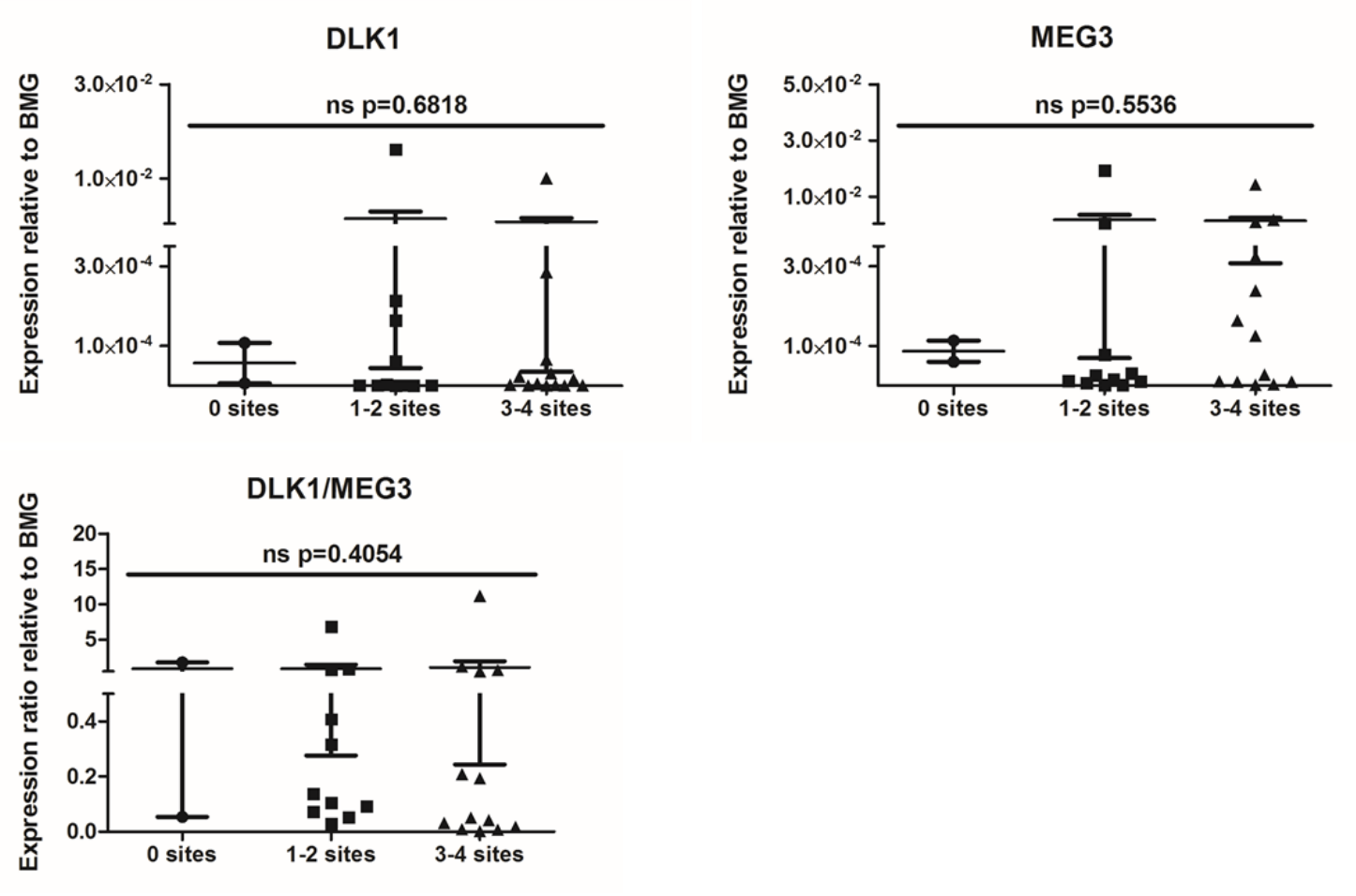

Figure 22. DLK1 and MEG3 expression is not associated with combined methylation results from CG6-9

Comparison of RT-qPCR results for human mononuclear cells from AML patients based on methylation at CG6-9 within the DLK1-MEG3 locus. Graphs represent the results of RT-qPCR performed once per sample in duplicate for each gene, where the average for each sample was used for analysis with the Kruskal-Wallis test and Dunn's Multiple Comparison Test based on the median methylation at CG6-9 for that sample, i.e. samples in the "0 sites" are from patients for whom the methylation at each $\mathrm{CpG}$ site was lower than the median methylation for that site, samples in the "1-2 sites" are from patients for whom the methylation at one or two $\mathrm{CpG}$ sites was higher than the median methylation for those sites, and samples in the "3-4 sites" are from patients for whom the methylation at three or four CpG sites was higher than the median methylation for those sites. DLKl, MEG3, DLKI/MEG3 - 0 sites $\mathrm{n}=2,1-2$ sites $\mathrm{n}=11,3-4$ sites $\mathrm{n}=13$. ns not significant 
DLK1

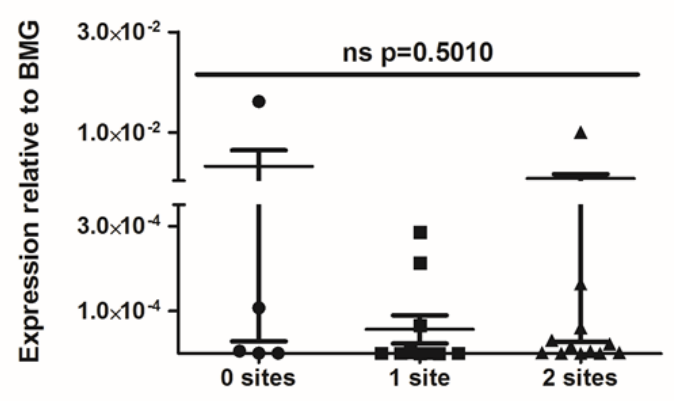

MEG3

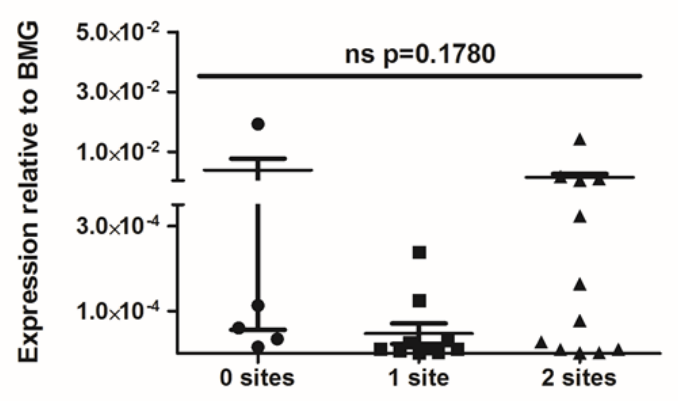

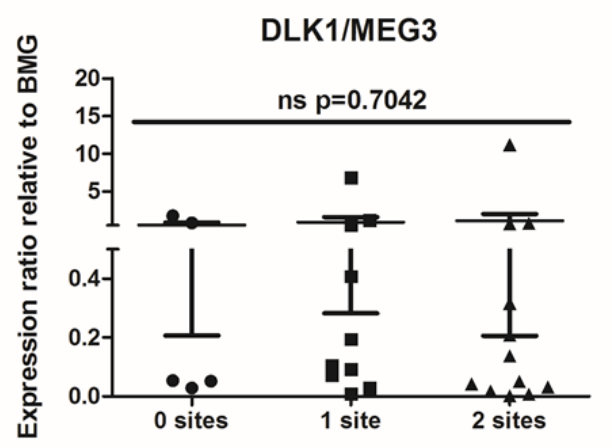

Figure 23. DLK1 and MEG3 expression is not associated with combined methylation results from CpG sites within the $M E G 3$ promotor region.

Comparison of RT-qPCR results for human mononuclear cells from AML patients based on methylation at CG7,9 within the DLK1-MEG3 locus. Graphs represent the results of RT-qPCR performed once per sample in duplicate for each gene, where the average for each sample was used for analysis with the Kruskal-Wallis test and Dunn's Multiple Comparison Test based on the median methylation at CG7,9 for that sample, i.e. samples in the "0 sites" are from patients for whom the methylation at each $\mathrm{CpG}$ site was lower than the median methylation for that site, samples in the "1 site" are from patients for whom the methylation at one $\mathrm{CpG}$ site was higher than the median methylation for that site, and samples in the " 2 sites" are from patients for whom the methylation at both $\mathrm{CpG}$ sites was higher than the median methylation for those sites. DLK1, MEG3, DLK1/MEG3 - 0 sites $\mathrm{n}=5,1$ site $\mathrm{n}=10,2$ sites $\mathrm{n}=12$. $\mathrm{ns}$ not significant 


\section{Discussion}

In this chapter I provide evidence that CpG sites within the DLK1-MEG3 locus are hypermethylated in AML patient samples, and that there are significant associations between patient survival and both $D L K 1$ expression and select $\mathrm{CpG}$ site methylation. Accumulating evidence continues to implicate the DLK1-MEG3 locus in the development of various human diseases, including those of the blood system [10, 60, 63, 104-110]. A recent landmark study described the functional requirements by long-term repopulating hematopoietic stem cells (LT-HSCs) for a large cluster of miRNAs located within the DlklMeg3 locus [6]. These miRNAs, hypothesized to originate from a large polycistronic transcriptional unit, were found to target members of the PI3K-mTOR pathway and restrict its overactivation [111]. Interestingly, deletion of the maternal IG-DMR repressed expression of $M e g 3$ and the miRNA-containing ncRNAs in murine fetal liver LT-HSCs, reduced the numbers of these cells, and upregulated $D l k 1$. Maternal deletion of the first 5 exons of $\mathrm{Meg} 3$ along with $~ 300 \mathrm{bp}$ of its promotor, corresponding to $\mathrm{CpG}$ site CG7 in my experiments and previously shown to similarly alter the expression of these genes in whole mouse embryos, also reduced the number of murine fetal liver LT-HSCs [7]. What's more, hypermethylation of the $M e g 3 \mathrm{DMR}$ and repressive chromatin marks at the $\mathrm{Meg} 3$ promotor were recently shown to accompany decreased expression of these miRNAs as the result of an induction process used to create induced pluripotent stem cells [4]. Taken together, these studies highlight the complexity of genomic imprinting at the IG-DMR and MEG3 DMR and its importance for the correct expression of growth-promoting and growth-suppressing genes in stem cells. 
Considering this, combined with the roles of DLK1 in the maintenance of normal and cancerous stem cells, it was not surprising to find that decreased AML patient survival was significantly associated with high $D L K 1$ expression. AML is characterized by the accumulation of blast cells in the bone marrow, and while the overexpression of $D L K 1$ was previously found to decrease the proliferation of human promyelocytic HL-60 cells, the differentiation of these cells was also inhibited [61]. DLK1 expression is also known to increase during the progression of MDS [63]. Thus, high expression of $D L K 1$ in leukemic blasts may promote their accumulation by blocking their differentiation and preventing their uptake of chemotherapeutics through hyperproliferation. This was not reflected in any association between $D L K 1$ expression and the complete remission of patients, but using a larger number of patients and separating them into "high DLKI expression" and "low DLK1 expression" groups may uncover such an association in future studies. More surprising, however, was the low expression of $D L K 1$ in AML cells compared to control MNCs. Previous studies have shown conflicting data regarding the expression of $D L K 1$ in AML patient samples compared to control MNCs $[60,112]$. These studies both utilized GAPDH as a control gene and normal human bone marrow for control MNCs, and inspection of their RT-qPCR primer sequences did not reveal any obvious issues. Because I utilized $B M G$ as a control gene and sourced MNCs from human umbilical cord blood, direct comparisons between my results and these studies cannot be made. However, discrepancies between these two studies could possibly be explained by the blast \% of MNCs isolated from AML and normal bone marrow. In addition, one study had a large percentage of patients which exhibited biallelic expression of $D L K 1$, which may have skewed their results towards higher $D L K 1$ expression overall. Interestingly, this study also 
found mono- and biallelic expression of $D L K 1$ to be under the control of a $\mathrm{CpG}$ site within the DLK1 promotor region in AML patients. In light of a recent report in which overexpression of the Meis gene in an ND13 murine model of AML resulted in the overexpression of $D l k l$ and hypermethylation of the IG-DMR, but not the Dlkl promotor, it could be valuable to check the expression of MEIS and biallelic expression of DLK1 in the patient samples I used [113]. Doing so could help explain the lack of associations I found between $\mathrm{CpG}$ site methylation and $D L K 1$ expression for these samples.

Like $D L K 1, M E G 3$ was found to be downregulated in AML samples compared to controls, but my results do not show any associations between $M E G 3$ expression and $\mathrm{CpG}$ site methylation for AML samples. Nor do they show associations between MEG3 expression and either the complete remission or survival of patients, which was unexpected due to the suppressive role previously found for $M E G 3$ in leukemogenesis [62]. Also like $D L K 1$, this could be explained by biallelic expression of $M E G 3$, which has not been thoroughly investigated in AML. While the average methylation of imprinted $\mathrm{CpG}$ sites within the DLK1-MEG3 locus was high for AML samples, at least one of these two CpG sites was hypomethylated for many patients. Similarly, the possibility of biallelic expression for these genes could also explain lack of associations between the DLK1/MEG3 expression ratio and either the outcome or patients or the methylation of $\mathrm{CpG}$ sites. Paradoxically, higher methylation at $\mathrm{CpG}$ sites within the MEG3 promotor region was significantly associated with increased survival by these patients. This conflicts with a previous report which found aberrant methylation to significantly associate with decreased AML patient survival, though this conflict is likely due to the method of methylation assessment [100]. However, given the growth-suppressing nature of MEG3 
and its downstream miRNAs and their dependence on the MEG3 DMR and promotor region for their correct expression, a decrease in miRNA expression would be expected for the samples which have higher methylation at these CpG sites $[6,7,98,114,115]$. This phenomenon could result in the overactivation of the PI3K-mTOR pathway, which is a common feature of AML, and allow for hyperproliferation of blasts and their enhanced uptake of chemotherapeutics. Thus, this would imply that the effect of increased $D L K 1$ expression and increased methylation of $\mathrm{CpG}$ sites within the $M E G 3$ promotor serve opposing roles in the survival of AML patients due to their effects on chemotherapeutic uptake. However, I did not find any association between methylation of these CpG sites and complete remission by patients. Previous studies of small ncRNA expression in AML uncovered miRNA signatures based on specific karyotypes and subtypes, with APL specifically upregulating several miRNAs and cell cycle-promoting small nucleolar RNAs (snoRNAs) from the DLK1-MEG3 locus[116]. Interestingly, while the overexpression of these snoRNAs was dependent on the PML-RARalpha fusion protein, the expression of specific miRNAs was associated with hypermethylation of CTCF binding motif-containing CpG sites corresponding to CG7, CG8, and CG9. Thus, it appears that miRNA expression from this locus can be positively associated with increased methylation, and the location of potential CTCF-binding sites within each of these $\mathrm{CpG}$ sites underscores the importance of 3-dimensional chromatin conformation and the complexity of gene expression regulation at this locus. Assessment of miRNA expression specifically from this locus may expose expression signatures which are associated specifically with increased survival.

Taken together, these results are the first to associate AML survival with the degree of $D L K 1$ expression and with the degree of methylation at the $M E G 3$ promotor. This is 
also one of the first instances where hypermethylation of $\mathrm{CpG}$ sites within the DLK1MEG3 locus appears beneficial for the patient, and this data may be useful for the future development of prognostic guidelines for AML. Future studies in search of miRNA expression signatures from the DLK1-MEG3 locus specifically associated with patient survival could offer further insight into the regulation of genes from this locus, and may serve as valuable biomarkers for AML.

\section{Conclusions}

- The initial screen of IGN DMR methylation uncovered differences between AML samples and control MNCs, though these differences did not amount to any significant associations between methylation and patient outcomes

- Similar to the initial IGN DMRs screened, significant differences in methylation at CpG sites within the DLK1-MEG3 locus were also found between AML patients and control MNCs. In contrast to the other IGN DMRs, however, methylation at select $\mathrm{CpG}$ sites within the $M E G 3$ promotor was significantly associated with AML patient survival.

- High DLK1 expression among AML patients was significantly associated with decreased patient survival, but no associations were found between patient outcomes and either $M E G 3$ expression or the $D L K 1 / M E G 3$ expression ratio. 
- Future studies integrating the epigenome with arrays of miRNA expression from the $D L K 1-M E G 3$ locus will provide valuable insight into gene regulation at this locus, and may aid in the future development of targeted therapies for AML. 


\section{CHAPTER 4: GENOMIC IMPRINTING IDENTIFIES DLK1 AS A NOVEL THERAPEUTIC TARGET FOR EMBRYONAL CARCINOMA}

\section{Introduction}

Primordial germ cells undergo widespread epigenomic changes during their maturation into sex-specific germ cells. Among these changes lies the complete erasure and reestablishment of genomic imprinting patterns [39]. Improper maintenance of genomic imprinting is found in several types of cancers and other growth-related diseases, yet few associations have been uncovered between aberrant imprinting and tumors of proposed PGC origins $[117,118]$. Chief among these associations identified thus far is hypomethylation at the IGF2-H19 locus [36-38]. While the participation of growth factors such as IGF2 in insulin-like signaling has been implicated in TGCT development, a recent study found the H19 lncRNA to be necessary for the maintenance of EC stem-like cells [18, 119].

Seminal work on the generation of bimaternal mice implicated two paternally imprinted loci as key barriers to this process: IGF2-H19 and DLK1-MEG3 [15, 120]. Later work determined that genetic manipulation of either locus was sufficient to carry pups through the majority of growth and development, but manipulation of both loci was necessary to efficiently create live, healthy mice $[15,17,121]$. Both loci harbor growthrelated protein-coding genes and growth-suppressing ncRNAs which are oppositely imprinted, such that 
the protein-coding genes are transcribed primarily from the paternally inherited chromosome, and the ncRNAs are transcribed primarily from the maternally inherited chromosome. Interestingly, lncRNAs from each locus, $H 19$ and $M E G 3$, are implicated in the suppression of teratoma growth $[19,20]$. What's more, ncRNAs from these loci help maintain the quiescence and stemness of HSCs, and hypomethylation of the IGF2-H19 locus is hypothesized to control the quiescence of adults pluripotent VSELs [5, 6, 8].

Several lines of evidence place HSCs and VSELs as derivatives of migrating PGCs in the developing embryo [42]. Similarly, TGCTs are proposed to originate from PGCs at various stages of their development [36]. Although PGCs and VSELs express pluripotency markers, neither population of cells is able to form teratomas in vivo or complement blastocyst development. On the other hand, EC cells can give rise to teratocarcinomas, and most TGCTs present with an EC component [54]. Interestingly, EC is placed atop the hierarchy of TGCT stemness and may be the stem-like cells which give rise to other TGCT tissues [43].

PGCs serve as the proposed origin of HSCs, VSELs, and TGCTs such as EC [4244]. Thus, it is interesting that TGCTs display such malignant growth while exhibiting hypomethylation at the IGF2/H19 ICR [36-38]. Surprisingly, H19 expression is necessary to maintain the stemness of EC cells [18]. However, no studies have investigated methylation at the IGF2-H19 ICR in human EC cells, which is important when considering the heterogeneity of tumor tissues. Given the importance of the Dlkl-Meg3 locus in the generation of bimaternal mice, combined with the roles of this locus in HSC maintenance, it is also surprising that imprinting at this locus has not been investigated in TGCTs or EC cells $[6,15,17]$. What's more, while tumor heterogeneity and the existence of CSCs in 
tumors is generally accepted now, mounting evidence has challenged the existence of a static phenotype for stem cells and cancer stem-like cells. The continuum of HSC stemness was recently associated with the abundance of imprinted ncRNAs expressed by each cell, promoting the question of whether genes from the DLK1-MEG3 or IGF2-H19 loci may help define CSCs [6]. As the CSCs of TGCTs, the importance of H19 for maintaining the stemness of EC cell lines underscores this possibility [18]. Taken together, I therefore hypothesized that genomic imprinting at the IGF2-H19 and DLK1-MEG3 loci promotes the malignant growth of EC cells.

\section{Results}

Hypomethylation at the paternally imprinted IGF2-H19 locus in NTera2 correlates with a low IGF2/H19 expression ratio

Multiple groups have confirmed a PGC-like hypomethylation of the IGF2-H19 ICR in GCT tissues [36, 38]. However, biallelic expression of $H 19$ and $I G F 2$ is also a common feature of TGCTs, and previous efforts to assess the biallelic expression of these genes in NTera2 were ineffective $[122,123]$. Here, I found that the IGF2-H19 ICR is hypomethylated in NTera2 by COBRA as well as by a more sensitive sequencing method (Figure 24A and 24B, respectively). In contrast, MNCs exhibit higher methylation at this locus, in agreement with a recent report on imprinting in human tissues [124].

Somatic-like methylation of the IGF2-H19 ICR corresponds to monoallelic expression of the H19 and IGF2 genes by a well-characterized CTCF binding motif, whereas hypomethylation of the ICR corresponds to biallelic expression of the $H 19$ gene [125]. As expected based on their respective IGF2-H19 ICR methylation values, I found 
that the IGF2/H19 expression ratio is significantly lower in NTera2 cells than MNCs (Figure 25A). Importantly, IGF2 expression as well as the $I G F 2 / H 19$ expression ratio are also significantly lower in NTera2 cells than in testes tissues, which were recently reported to carry somatic methylation at the IGF2-H19 ICR, suggesting that autocrine IGF2 signaling may not be an important feature of NTera2 growth [124] 
A.

\begin{tabular}{|c|c|c|c|}
\hline & NTera2 & Humal & MNCs \\
\hline & $\overline{(-)}(+)$ & $(-) \quad(+)$ & $(-)$ \\
\hline IGF2-H19 DMR & -0 & $=$ & $=$ \\
\hline $231 \mathrm{BP}$ & & & \\
\hline 3 cut sites & $18 \% \mathrm{M}$ & $72 \% \mathrm{M}$ & $62 \% \mathrm{M}$ \\
\hline
\end{tabular}

B.

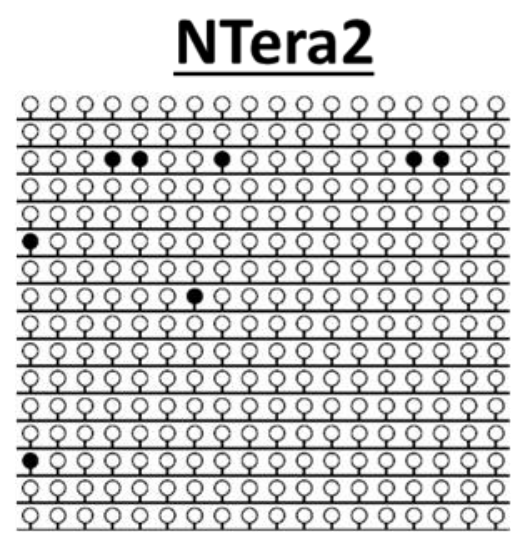

$3 \% \mathrm{M}$

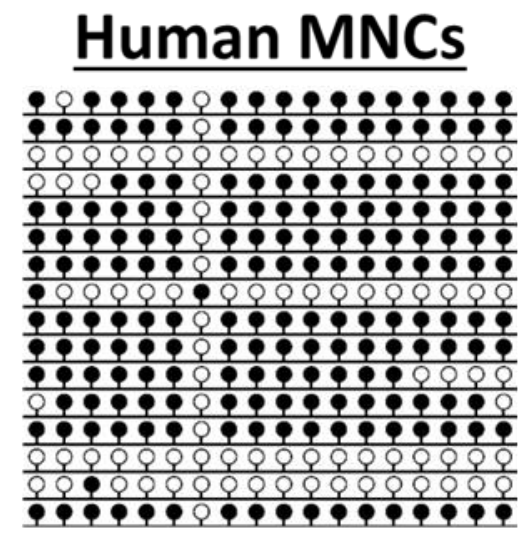

$68 \% \mathrm{M}$

Figure 24. NTera2 cells exhibit hypomethylation at the IGF2-H19 ICR

(A) Analysis of BSgDNA amplicons by COBRA. Densitometric analysis of undigested amplicons (-) and amplicons digested by a restriction enzyme (+) was used to estimate the methylation percentage $(\% \mathrm{M})$ at the IGF2-H19 ICR. (B) Sequencing of BSgDNA amplicons from the IGF2-H19 ICR was used to confirm the COBRA results for this DMR. Graphs represent the results of one experiment. 
A.

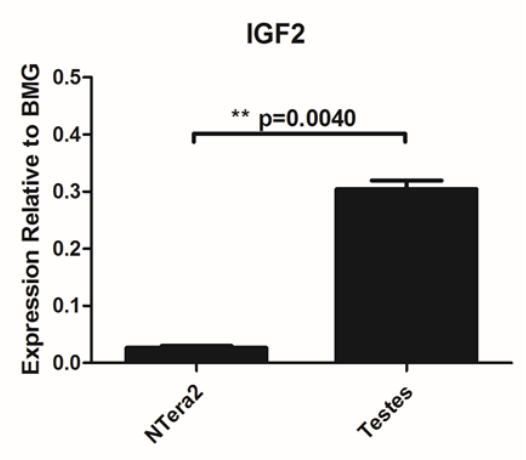

IGF2/H19

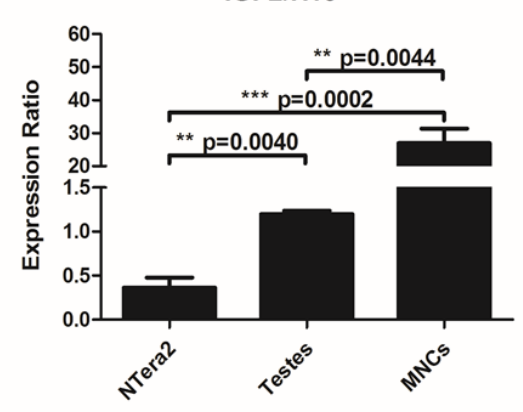

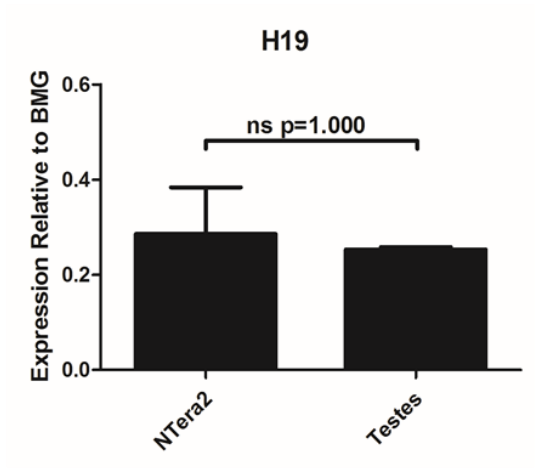

B.

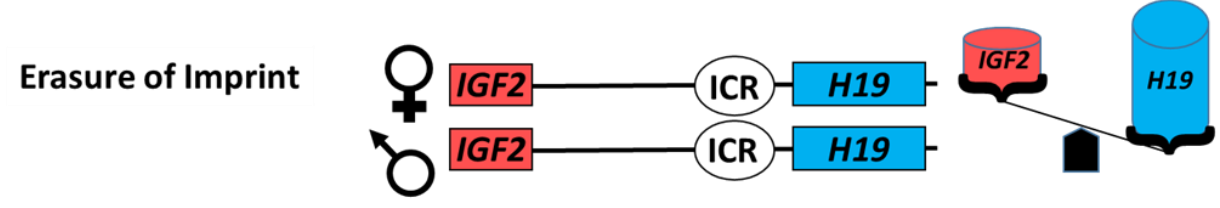

Figure 25. A low IGF2/H19 expression ratio in NTera2 cells reflects their erasure of imprinting at this locus

(A) RT-qPCR analysis of gene expression from the IGF2-H19 locus. Graphs represent the results of two experiments carried out in duplicate and analyzed using the Mann-Whitney $\mathrm{U}$ test. (B) Methylation and expression data from the IGF2-H19 locus demonstrate an erasure of imprint for this locus in Ntera2 cells. Hypomethylation at the ICR (unfilled oval) on each chromosome results in decreased expression IGF2 relative to H19. ${ }^{*} \mathrm{p}<0.01$ $* * * \mathrm{p}<0.001$ 


\section{Hypermethylation at the paternally imprinted DLK1-MEG3 locus in NTera2}

correlates with a high DLK1/MEG3 expression ratio

In contrast to the IGF2-H19 ICR, no studies have directly investigated the imprinting pattern of the DLK1-MEG3 IG-DMR in human GCTs. However, hypermethylation of the MEG3 promotor was recently documented in TGCT tissues and cell lines, warranting further investigation into the methylation status of DMRs within this locus [126]. I found by COBRA that the DLKI-MEG3 IG-DMR and the MEG3 DMR are both hypermethylated in NTera2 cells compared to MNCs (Figure 26A), and I confirmed these results for the IG-DMR by a more sensitive sequencing method (Figure 26B).

Though the exact mechanism of imprinting at the DLK1-MEG3 locus has yet to be determined, the association of methylation at the IG-DMR and MEG3 DMR is similar to that of the IGF2-H19 locus [99]. I found that the DLK1/MEG3 expression ratio is significantly higher in NTera2 cells than MNCs, in agreement with their respective methylation patterns at both DMRs within this locus (Figure 27A). Interestingly, while the expression of $D L K 1$ is significantly lower in NTera2 cells than in testes, the $D L K 1 / M E G 3$ ratio is significantly higher in NTera2 cells, suggesting that methylation patterns within this locus may promote the malignant growth of NTera2. This expression ratio agrees with a recent report which found somatic methylation at the DLK1-MEG3 IG-DMR in human testes tissues [124]. What's more, immunostaining of NTera2 cells revealed a strong presence of DLK1 in the nuclei of these cells (Figure 28). This is a surprising observation, as studies with DLK1 have primarily focused on its roles in growth and differentiation for membrane-bound and soluble DLK1 proteins only, with few studies commenting on its presence in the nucleus [127-130]. 
A.

\begin{tabular}{|c|c|c|}
\hline & MW (-) (+) & $\frac{\text { Human MNCs }}{(-)(+)(-)(+)}$ \\
\hline $\begin{array}{l}\text { DLK1-MEG3 IG-DMR } \\
429 \text { BP } \\
3 \text { cut sites }\end{array}$ & $99 \% \mathrm{M}$ & $68 \%$ M 77\% M \\
\hline $\begin{array}{l}\text { MEG3 DMR } \\
332 \text { BP } \\
3 \text { cut sites }\end{array}$ & 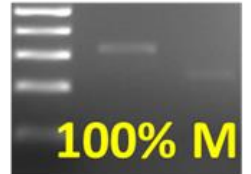 & $61 \% \mathrm{M} 47 \% \mathrm{M}$ \\
\hline
\end{tabular}

B.

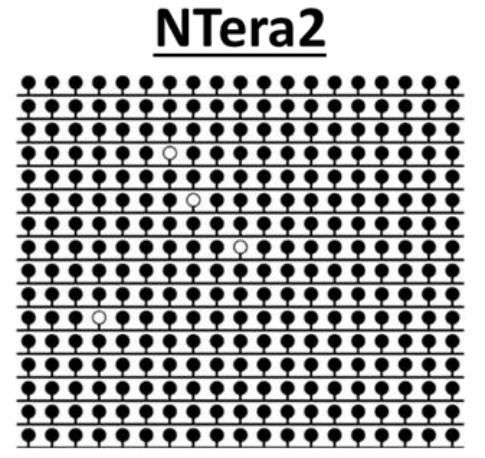

$99 \% \mathrm{M}$

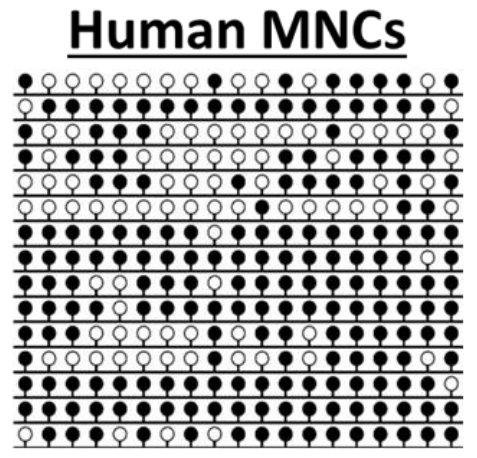

$69 \% \mathrm{M}$

Figure 26. NTera2 cells exhibit hypermethylation at DMRs within the DLK1-MEG3 locus

(A) Analysis of BSgDNA amplicons by COBRA. Densitometric analysis of undigested amplicons (-) and amplicons digested by restriction enzymes (+) was used to estimate the methylation percentage $(\% \mathrm{M})$ at the DLK1-MEG3 IG-DMR and MEG3 secondary DMR.

(B) Sequencing of BSgDNA amplicons from the DLK1-MEG3 IG-DMR was used to confirm the COBRA results for this DMR. Graphs represent the results of one experiment. 
A.

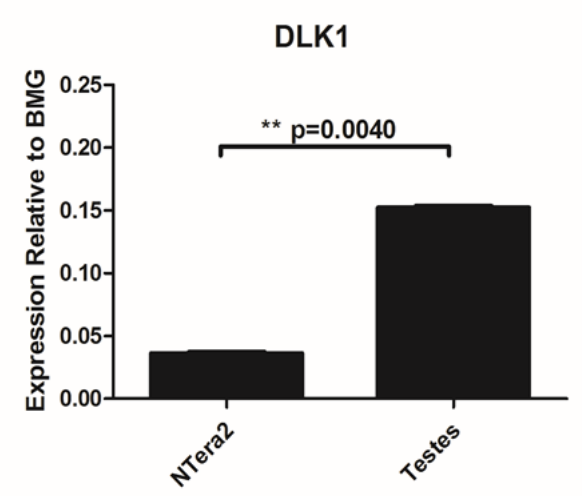

DLK1/MEG3

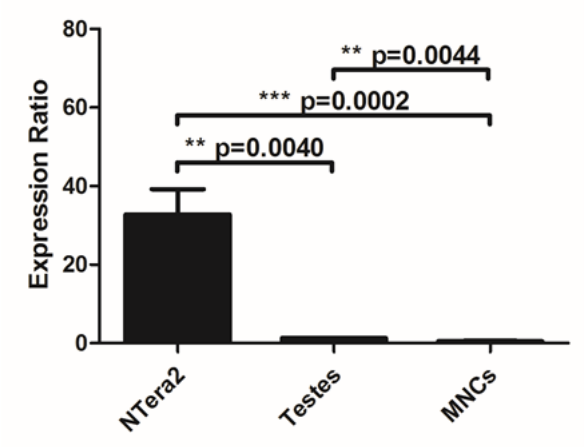

MEG3

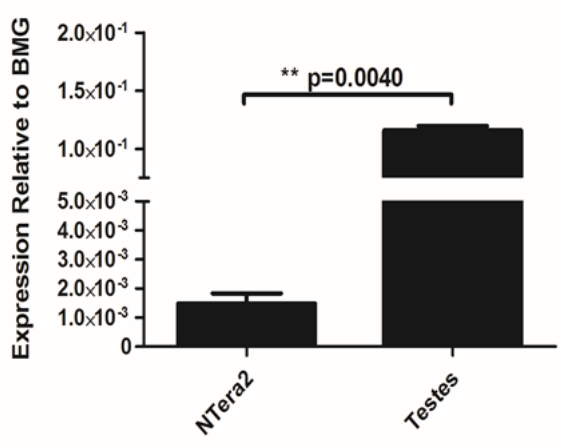

B.

Loss of Imprint
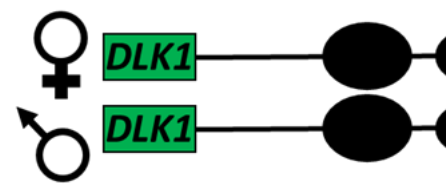

MEG3

MEG3

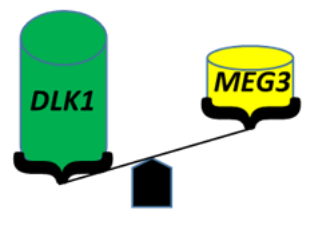

Figure 27. A high DLK1/MEG3 expression ratio in NTera2 cells reflects their loss of imprinting at this locus

(A) RT-qPCR analysis of gene expression from the DLK1-MEG3 locus. Graphs represent the results of two experiments carried out in duplicate and analyzed using the MannWhitney $\mathrm{U}$ test. (D) Methylation and expression data from the DLK1-MEG3 locus demonstrate a loss of imprint for this locus in Ntera2 cells. Hypermethylation at the IGDMR (large filled oval) and MEG3 secondary DMR (small filled oval) on each chromosome results in increased expression of $D L K 1$ relative to $M E G 3$. **p $<0.01$ $* * * \mathrm{p}<0.001$ 


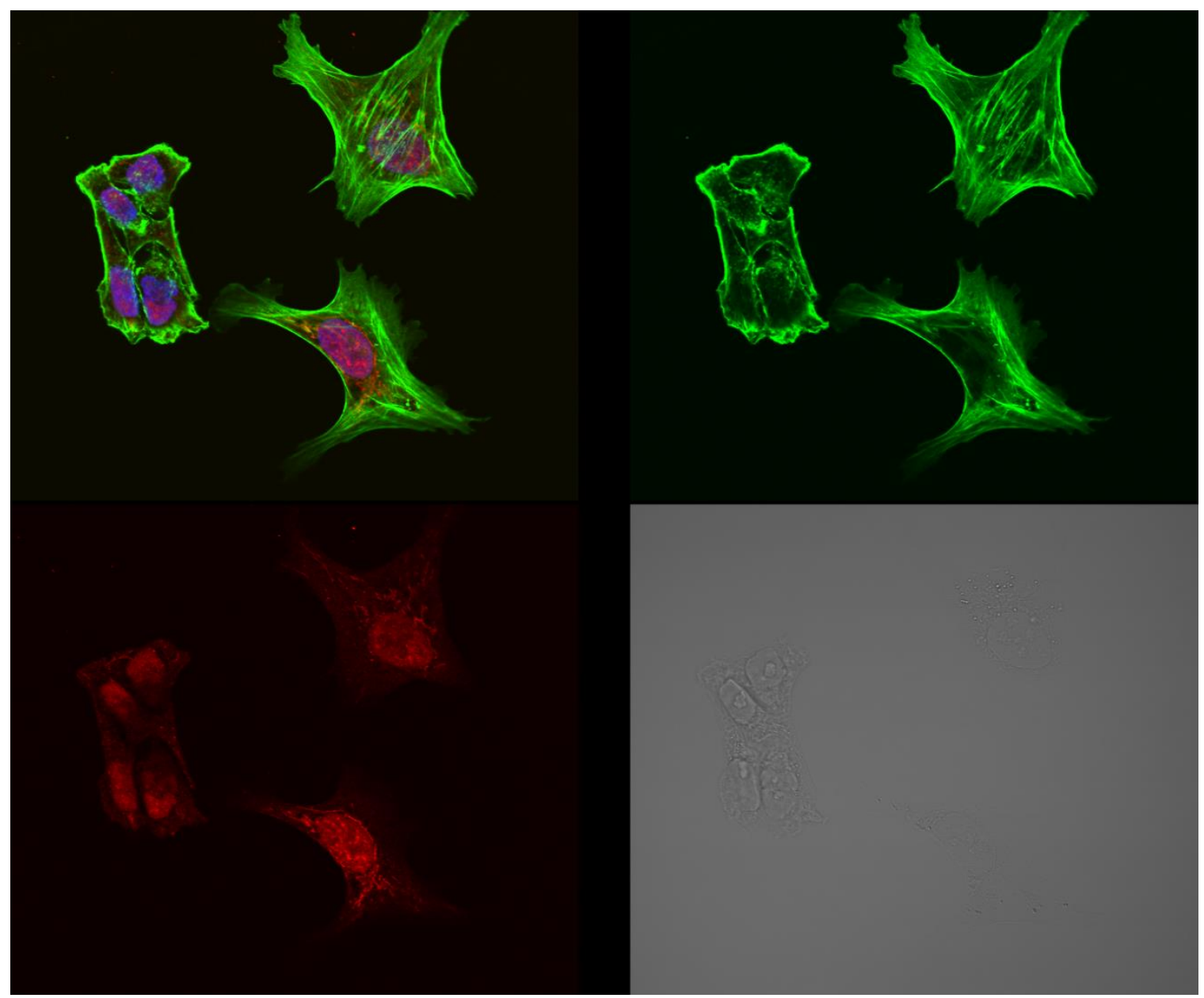

Figure 28. NTera2 cells exhibit a strong nuclear presence of the DLK1 protein

Representative confocal 3D overlay of NTera2 cells showing the nucleus (DAPI; blue), Factin (green), DLK1 (red), and brightfield. 
The demethylating compound 5-aza-2'-deoxycytidine inhibits the in vitro growth of NTera2 while demethylating the MEG3 promotor and augmenting the DLK1/MEG3 and IGF2/H19 expression ratios

The toxicity of the demethylating compound 5-aza-2'-deoxycytidine (5-azaD) to EC cells has been documented by multiple studies $[52,53]$. The 5-azaD analog 5azacytidine (5-azaC) was recently shown to demethylate the IGF2-H19 ICR in human rhabdomyosarcoma cells and augment their IGF2/H19 expression ratio [131]. Because 5azaD can prevent de novo DNA methylation and cause DNA methyltransferases to covalently bind DNA, I investigated the potential of 5-azaD to augment gene transcription from the IGF2-H19 and DLK1-MEG3 loci. In agreement with previous reports, I found that nanomolar doses of 5-azaD decreased the in vitro proliferation rate of NTera2 cells (Figure 29A). Because the IGF2-H19 ICR is already hypomethylated in NTera2 cells, I investigated the ability of 5-azaD to demethylate the DLK1-MEG3 locus (Figure 29B). Interestingly, while methylation of the $M E G 3$ promotor decreased in response to 5-azaD treatment, the IG-DMR methylation status remained unchanged.

As expected with demethylation at the $M E G 3$ promotor, $M E G 3$ expression was significantly increased in 5-azaD-treated cells (Figure 30). Interestingly, although no changes were observed in IG-DMR methylation, $D L K 1$ expression was also significantly decreased in treated cells. These changes in MEG3 and $D L K 1$ expression resulted in a significant decrease in the DLK1/MEG3 ratio, which became evident by Day 3 of the treatment. Moreover, while the IGF2-H19 ICR is already hypomethylated in NTera2 cells, significant changes in $I G F 2$ and $H 19$ expression along with a significant increase in the $I G F 2 / H 19$ expression ratio were also found in treated cells (Figure 31 ). While 5-azaD is a 
DNA methylation inhibitor, its toxicity is attributed to its ability to cause covalent attachment of DNA methyltransferases to DNA [132]. This is important when considering the proposed CTCF-governed control of gene expression at these loci, as covalent adducts are likely to inhibit CTCF binding to DNA. In addition, methylation changes at the IGF2 promotor and the intragenic IGF2 DMR may also play roles in the expression of this gene [133]. Similarly, a conserved CTCF binding site is located within the intragenic portion of the $M E G 3$ promotor region, which may also affect the DLK1/MEG3 expression ratio [134]. Thus, 5-azaD treatment of NTera2 cells results in a significant decrease in their in vitro proliferation rate, and this is accompanied by $M E G 3$ promotor demethylation and likely other aspects of genomic imprinting disruption. Most importantly, this decrease in the proliferation rate of treated cells is accompanied by a significant decrease in their DLK1/MEG3 expression ratio. This decrease in the proliferation rate of treated cells also occurs despite their increased IGF2 expression and increased IGF2/H19 expression ratio, with no statistically significant changes in the $I G F I R$ expression of these cells (Figure 32). These results indicate that the maintenance of expression ratios from the IGF2-H19 and DLK1-H19 loci is associated with the proliferative capacity of NTera2 cells, and suggest that the DLK1/MEG3 expression ratio is more important than the $I G F 2 / H 19$ expression ratio in the proliferation of NTera2. It is also interesting that OCT4 expression was decreased by 5 -azaD treatment, suggesting the DLK1/MEG3 ratio may be associated with OCT4 expression.

It is worth noting that in order to be sure the IGF2-H19 and DLK1-MEG3 loci were worth investigating in EC, I screened methylation at the DMRs of several maternally imprinted loci (Figure 33). Each of these loci exhibited either hypomethylation or somatic- 
like methylation, with one exception (Figure 34). The P57-LIT1 locus was slightly hypermethylated in comparison to MNCs and its P57/LIT1 expression ratio reflected this imprint (Figure 35). However, while the exact roles of LIT1 are not very well characterized, P57 encodes a cell cycle inhibitor, in contrast to the paternally expressed growth-promoting IGF2 and DLK1 genes. In addition, 5-azaD decreased the expression of P57 while inhibiting the proliferation of NTera2 cells, suggesting that P57 does not play a significant role in the proliferation of NTera2 (Figure 36). Thus, I concluded that this locus was likely not as important for the malignancy of NTera 2 as the IGF2-H19 or the DLK1-MEG3 loci, and continued my investigation with that focus. 
A.

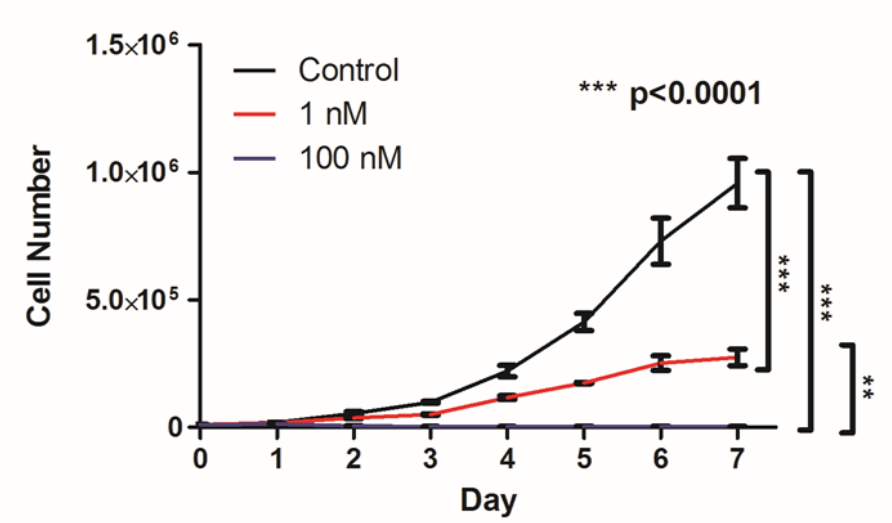

B.

Control

5-azaD

Day 3 Day 7 Day 3 Day 7

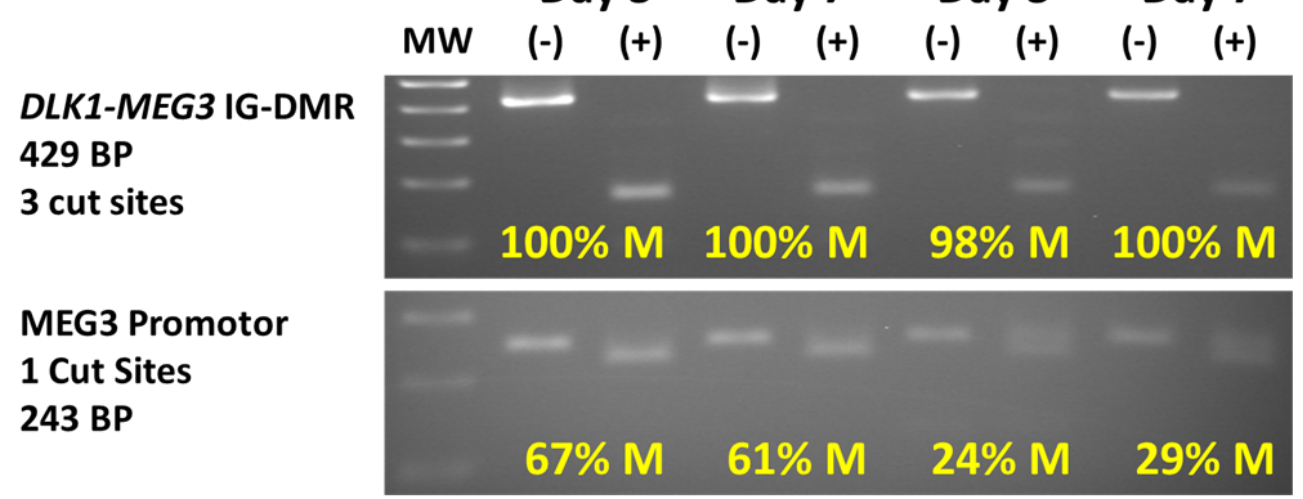

Figure 29. 5-azaD treatment of NTera2 cells inhibits their proliferation and demethylates the $M E G 3$ promotor

(A) Proliferation assay of NTera2 cells treated with 5-azaD. Graph represents the results of two experiments carried out in triplicate, where Day 6 cell numbers were analyzed using a one-way ANOVA with a Tukey post-test. (B) Analysis of BSgDNA amplicons by COBRA. Densitometric analysis of undigested amplicons (-) and amplicons digested by restriction enzymes (+) was used to estimate the methylation percentage $(\% \mathrm{M})$ at the DLK1-MEG3 IG-DMR and MEG3 secondary DMR for NTera2 cells treated with $100 \mathrm{nM}$ 5 -azaD for 3 and 7 days. Each graph represents the results of one experiment. $* * p<0.01$ $* * * \mathrm{p}<0.001$ 
DLK1 Day 3

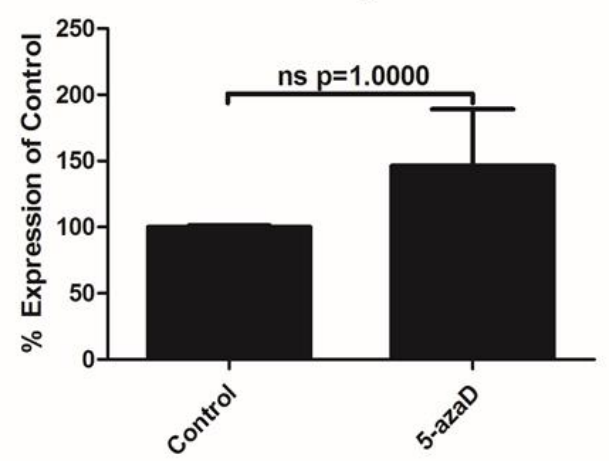

MEG3 Day 3
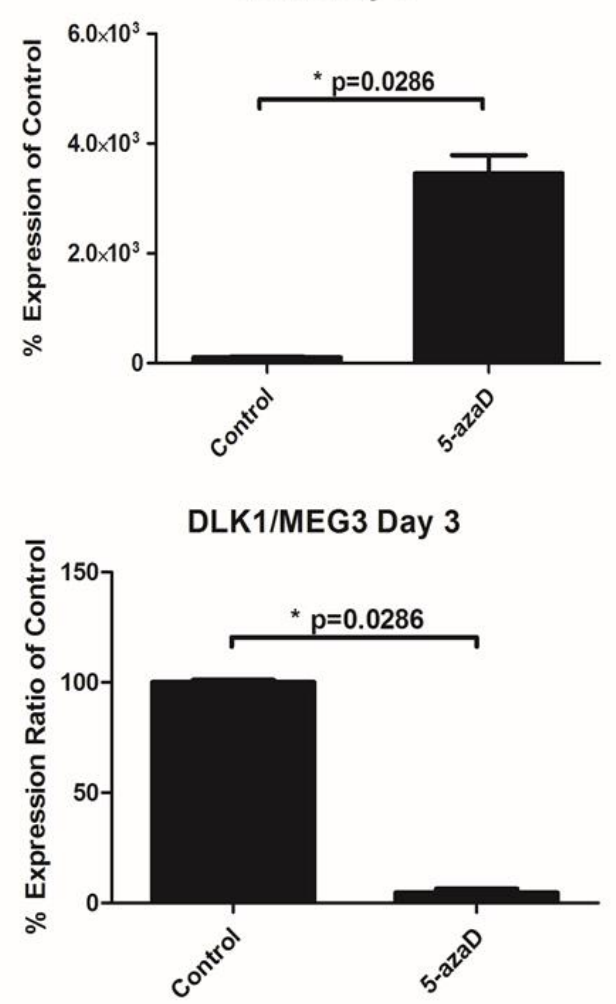

DLK1 Day 7

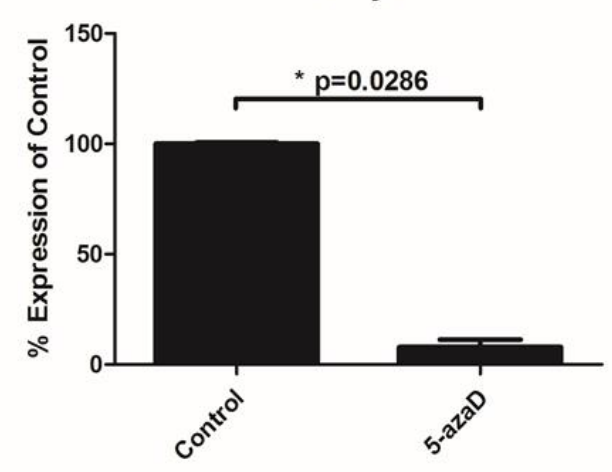

MEG3 Day 7
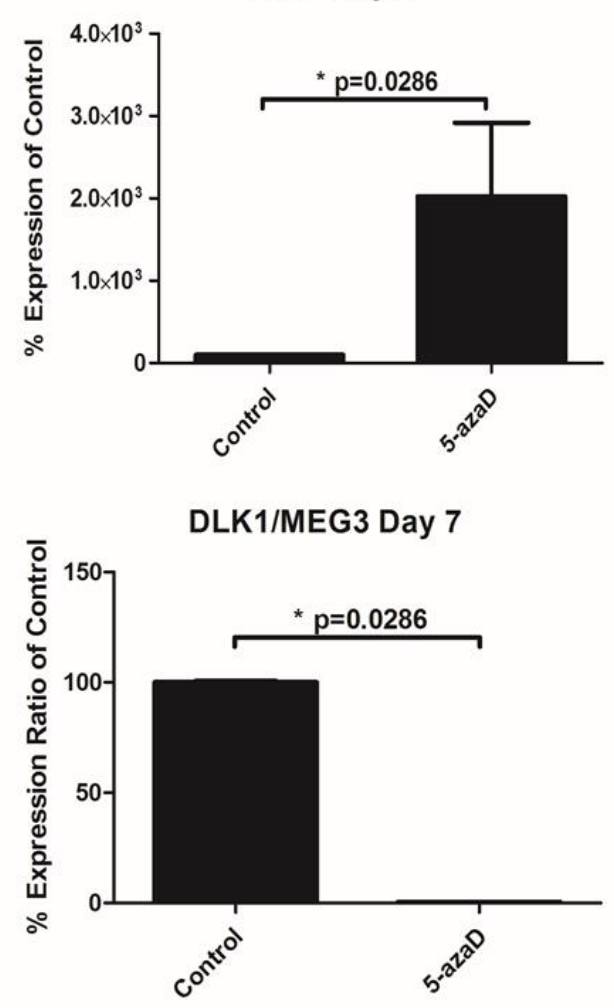

Figure 30. 5-azaD treatment of NTera2 cells augments their expression of genes from the DLK1-MEG3 locus

RT-qPCR analysis of gene expression from the DLK1-MEG3 locus in cells treated with $100 \mathrm{nM} 5$-azaD for 3 and 7 days. Graphs represent the results of two experiments carried out in duplicate and analyzed using the Mann-Whitney $U$ test. $* \mathrm{p}<0.05$ 
IGF2 Day 3

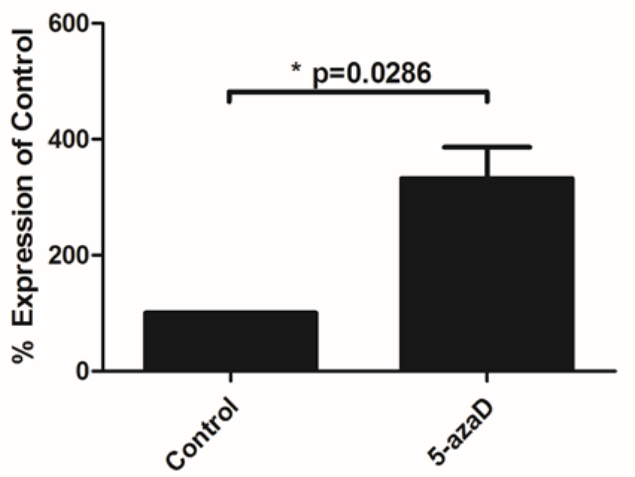

H19 Day 3

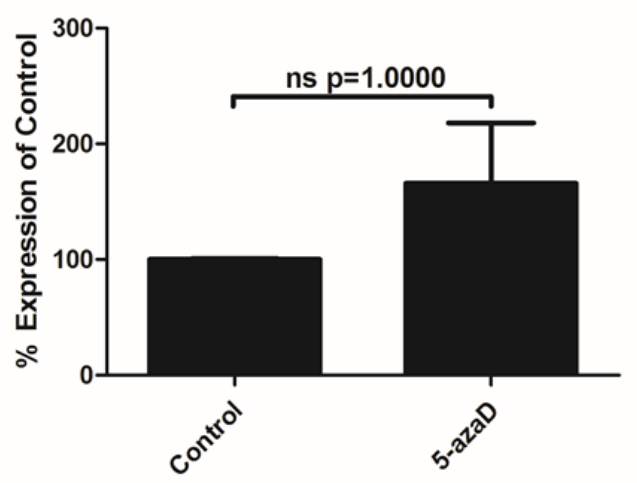

IGF2/H19 Day 3

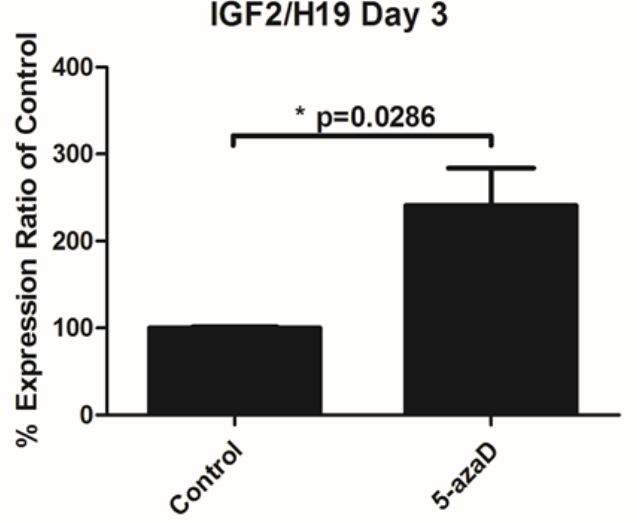

IGF2 Day 7

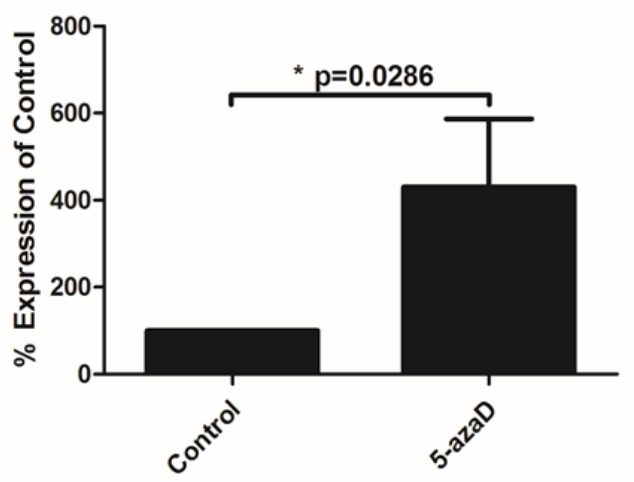

H19 Day 7
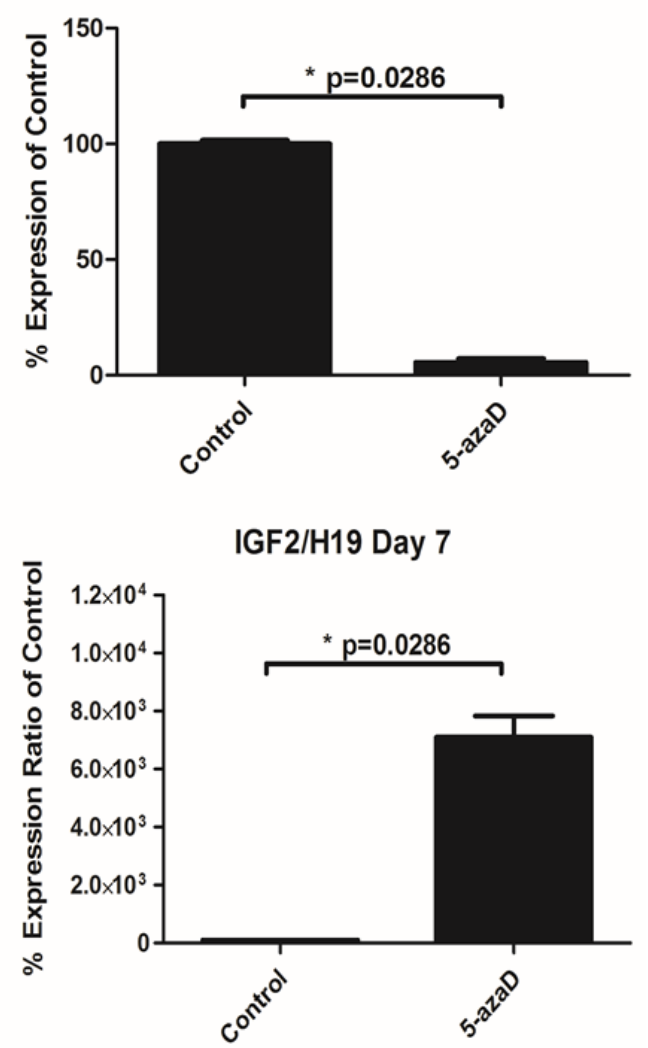

Figure 31. 5-azaD treatment of NTera2 cells augments their expression of genes

\section{from the IGF2-H19 locus}

RT-qPCR analysis of gene expression from the IGF2-H19 locus in cells treated with 100 nM 5-azaD for 3 and 7 days. Graphs represent the results of two experiments carried out in duplicate and analyzed using the Mann-Whitney U test. *p $<0.05$ 

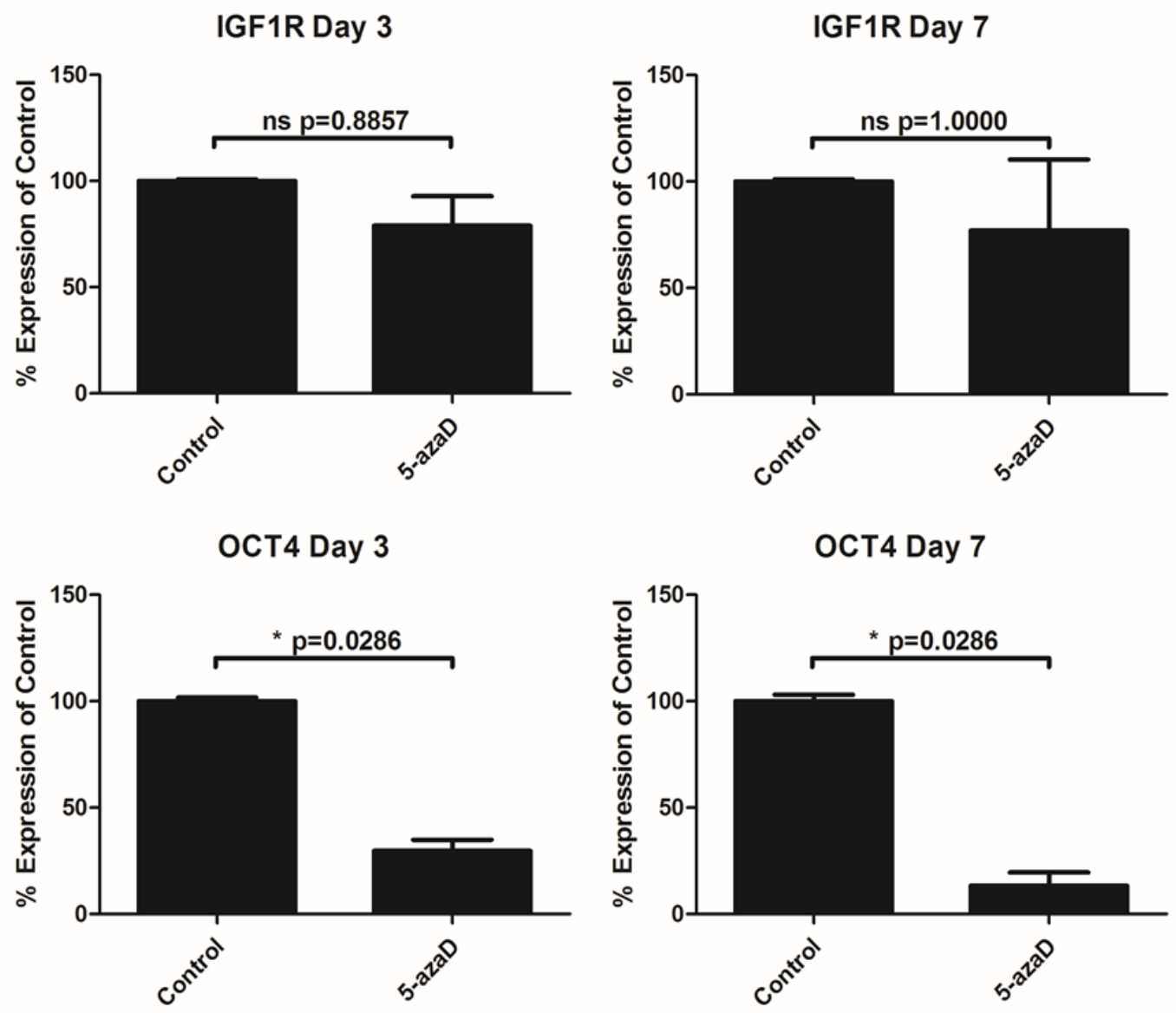

Figure 32. 5-azaD treatment of NTera2 cells decreases their expression of the pluripotency marker $O C T 4$

RT-qPCR analysis of gene expression in cells treated with $100 \mathrm{nM} 5$-azaD for 3 and 7 days. Graphs represent the results of two experiments carried out in duplicate and analyzed using the Mann-Whitney U test. ${ }^{*} \mathrm{p}<0.05$ 


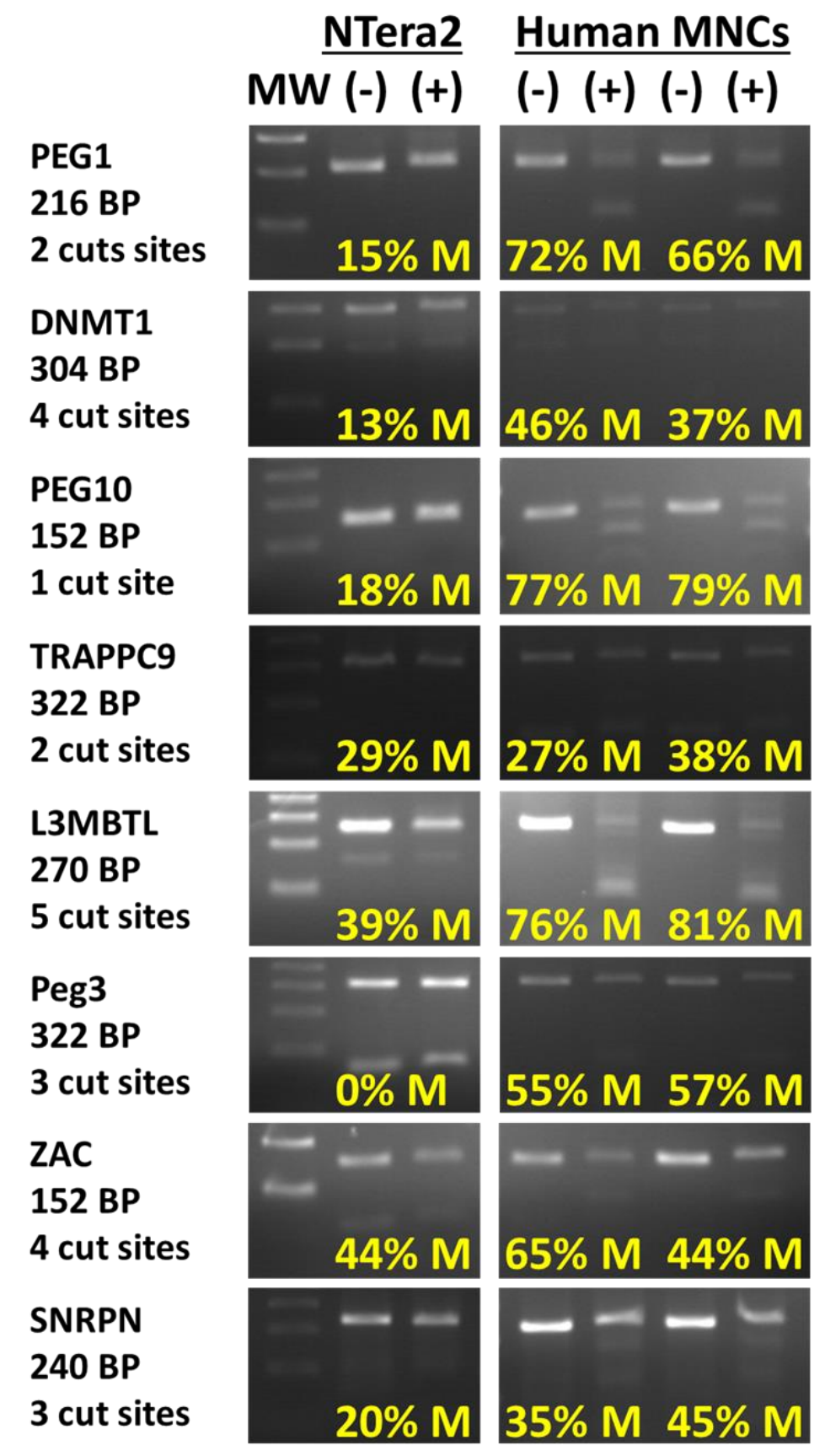

Figure 33. NTera2 cells exhibit low methylation at maternally imprinted loci Analysis of BSgDNA amplicons by COBRA. Densitometric analysis of undigested amplicons (-) and amplicons digested by restriction enzymes (+) was used to estimate the methylation percentage $(\% \mathrm{M})$ at the DMRs of maternally imprinted loci. Each graph represents the results of one experiment. 
A.

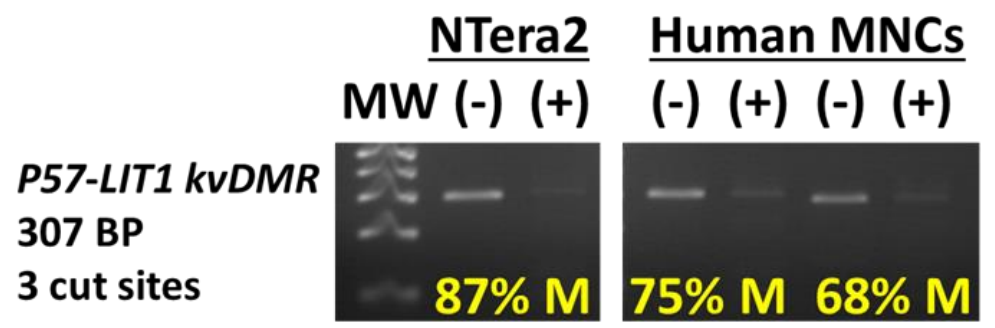

B.

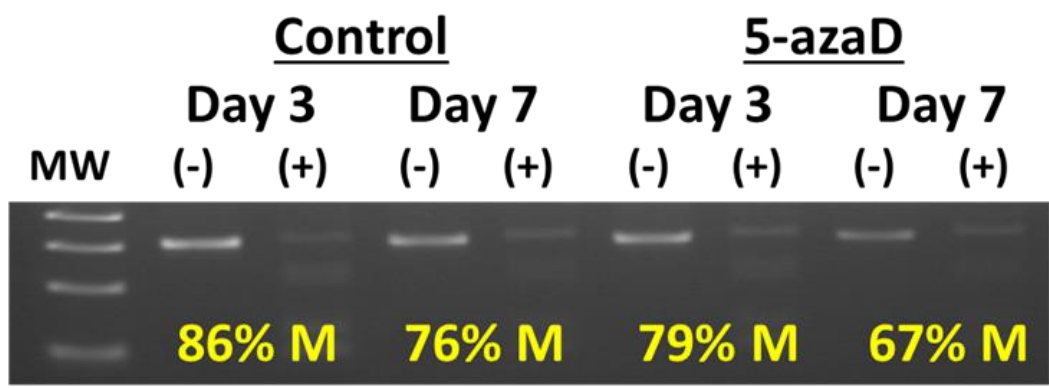

Figure 34. NTera2 cells exhibit hypermethylation at the P57-LIT1 locus, which is slightly demethylated by 5-azaD

Analysis of BSgDNA amplicons by COBRA. Densitometric analysis of undigested amplicons (-) and amplicons digested by restriction enzymes (+) was used to estimate the methylation percentage (\% M) at the P57-LIT1 KvDMR for (A) NTera2 cells and MNCs under normal conditions and (B) NTera2 cells treated with $100 \mathrm{nM}$ 5-azaD for 3 and 7 days. Each graph represents the results of one experiment. 
A.
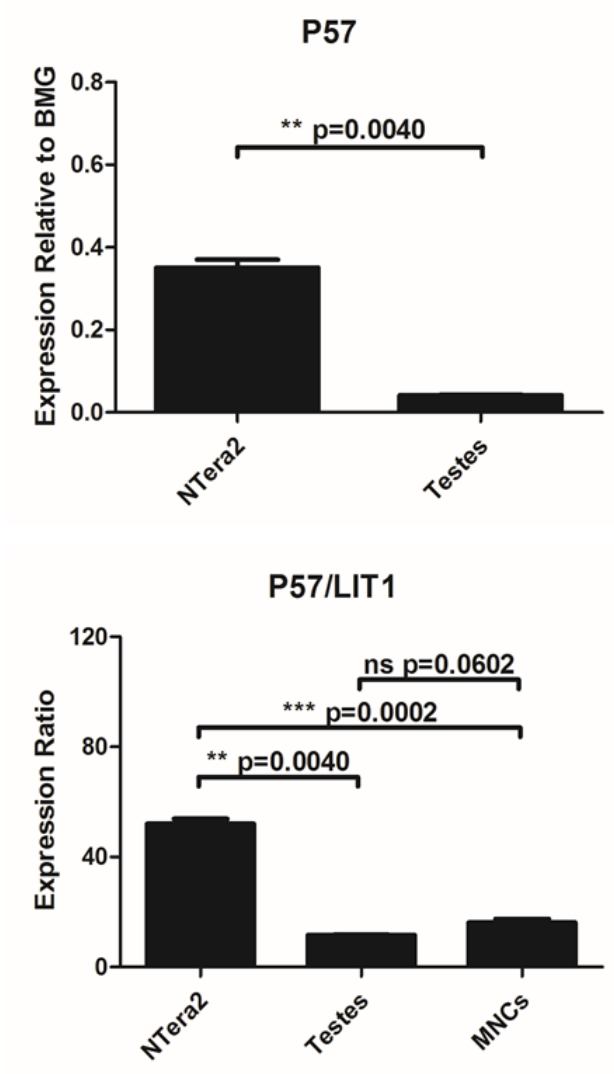

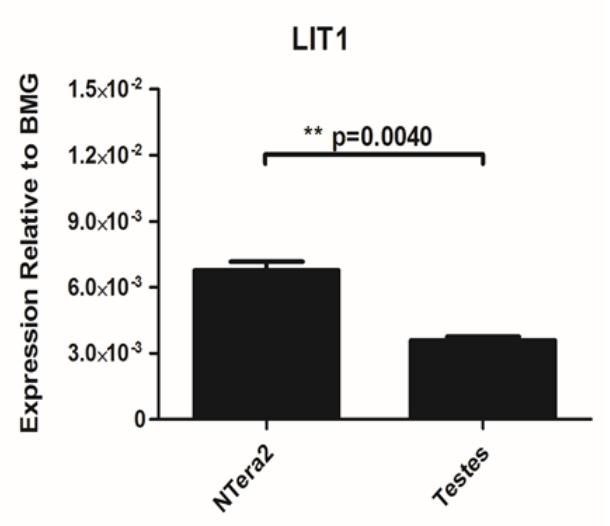

B.

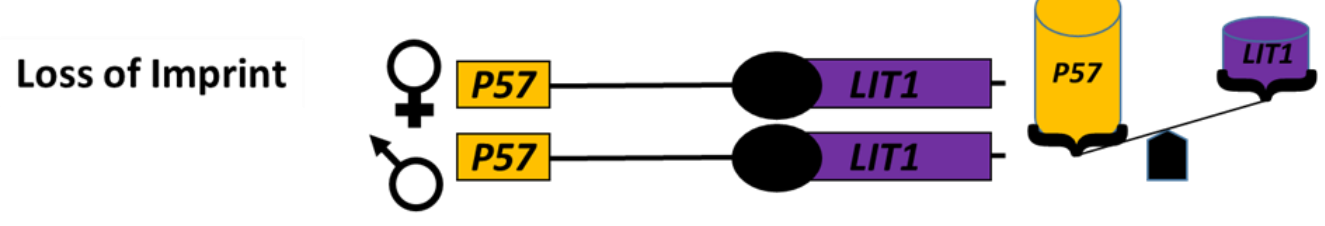

Figure 35. A high P57/LIT1 expression ratio in NTera2 cells reflects their loss of imprinting at this locus

(A) RT-qPCR analysis of gene expression from the P57-LIT1 locus. Graphs represent the results of two experiments carried out in duplicate and analyzed using the Mann-Whitney U test. (B) Methylation and expression data from the P57-LIT1 locus demonstrate a loss of imprint for this locus in Ntera2 cells. Hypermethylation at the KvDMR (large filled oval) on each chromosome results in increased expression of P57 relative to LIT1. ns not significant $* * \mathrm{p}<0.01 * * * \mathrm{p}<0.001$ 
P57 Day 3

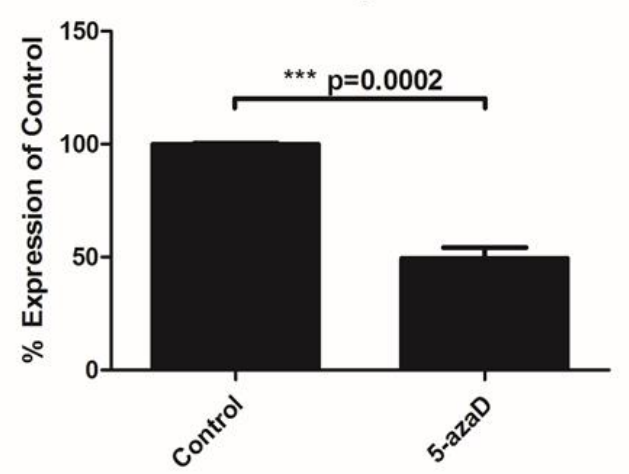

LIT1 Day 3

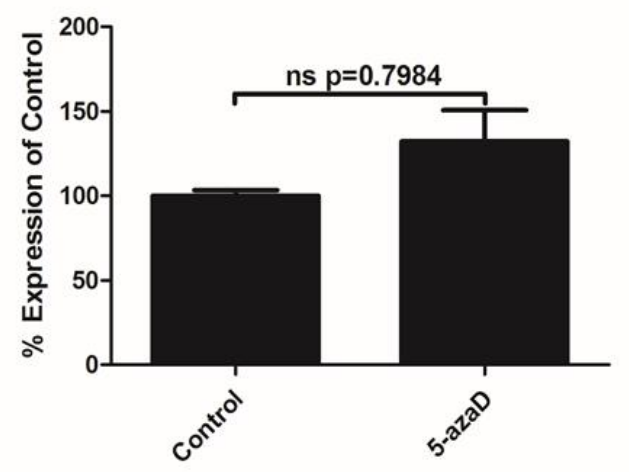

P57/LIT1 Day 3

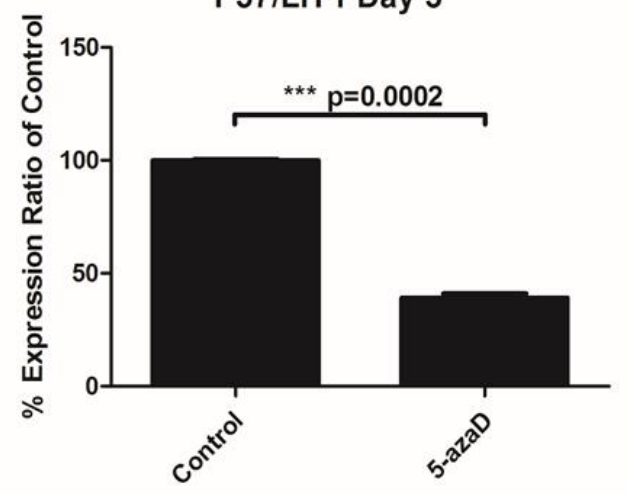

P57 Day 7

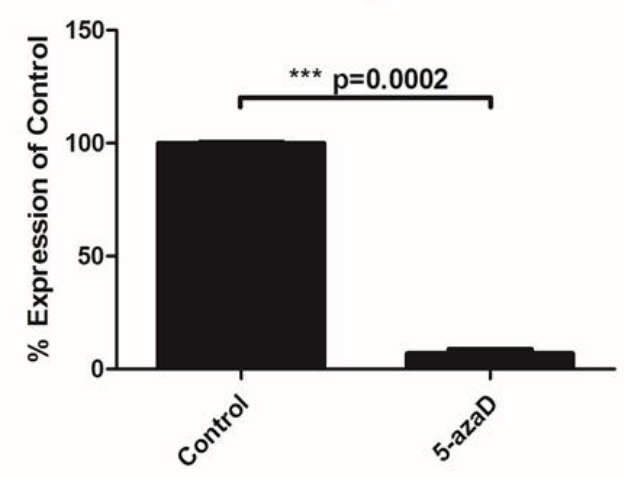

LIT1 Day 7

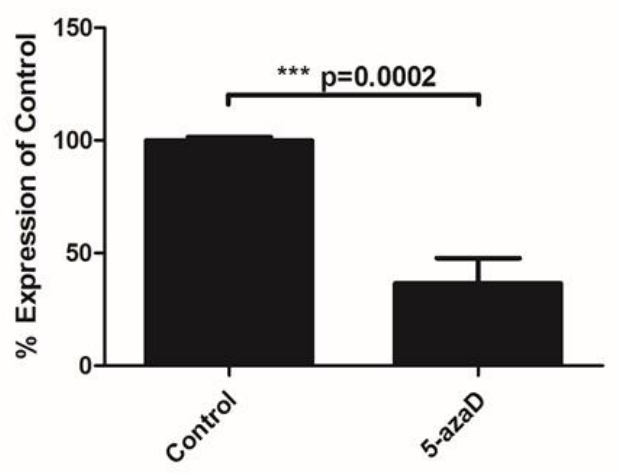

P57/LIT1 Day 7

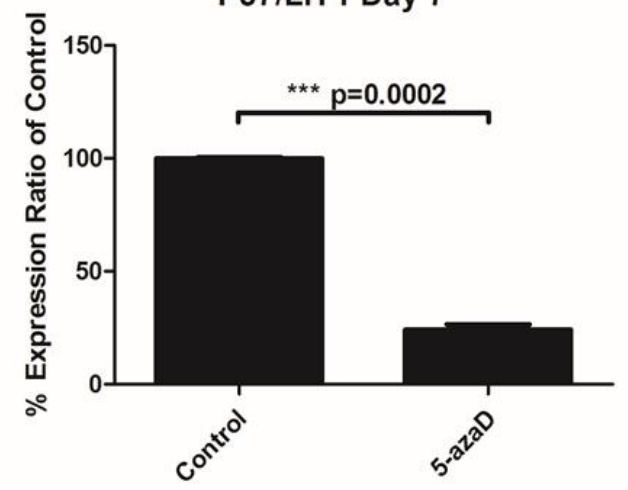

Figure 36. 5-azaD treatment of NTera2 cells augments their expression of genes

\section{from the P57-LIT1 locus}

RT-qPCR analysis of gene expression from the P57-LIT1 locus in NTera2 cells treated with $100 \mathrm{nM} 5$-azaD for 3 and 7 days. Graphs represent the results of two experiments carried out twice in duplicate and analyzed using the Mann-Whitney $U$ test. ${ }^{*} * * p<0.001$ 


\section{NTera2 expresses insulin-like growth factor-related genes}

Insulin-like signaling involves several surface receptors and signaling peptides

(Figure 4). Previous studies have found insulin-like signaling to be important for the growth of human murine teratocarcinoma, and it is proposed to be involved in TGCT development [119, 135-137]. Because 5-azaD treatment of NTera2 decreased the proliferation rate of these cells despite increasing their relatively low $I G F 2$ expression and IGF2/H19 expression ratio, I investigated the expression of other insulin-like signaling genes by NTera2 cells to better characterize the capacity for insulin-like signaling in these cells. I found that NTera 2 cells have significantly lower IGFI expression, but significantly higher expression of IGFIR and INSR, than testes (Figure 37A), and I verified the expression of IGF1R and INSR by NTera2 cells at the protein level by flow cytometry analysis (Figure 37B). The high expression of insulin-like signaling receptors by NTera2 cells indicates that, like TGCT tissues, these cells may also be responsive to insulin-like signaling growth factors. Also, like IGF2, NTera2 cells also express significantly less $I G F 1$ than testes, suggesting that autocrine insulin-like signaling is not important for these cells. 
A.
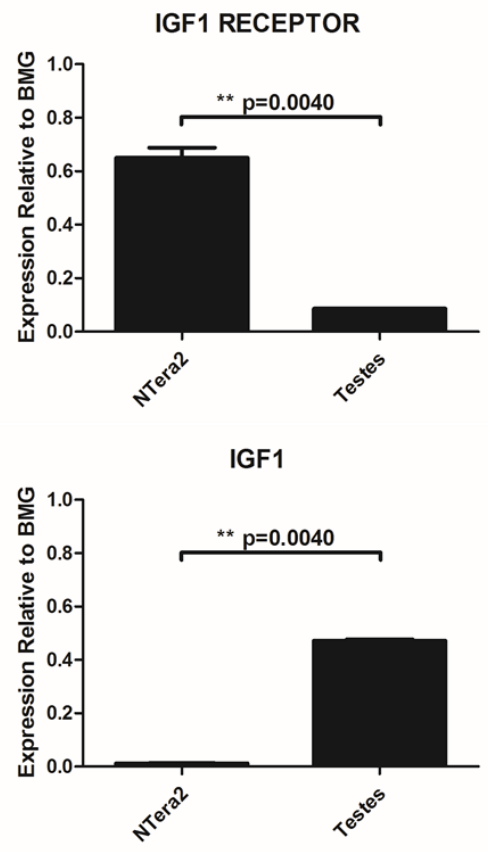

B.
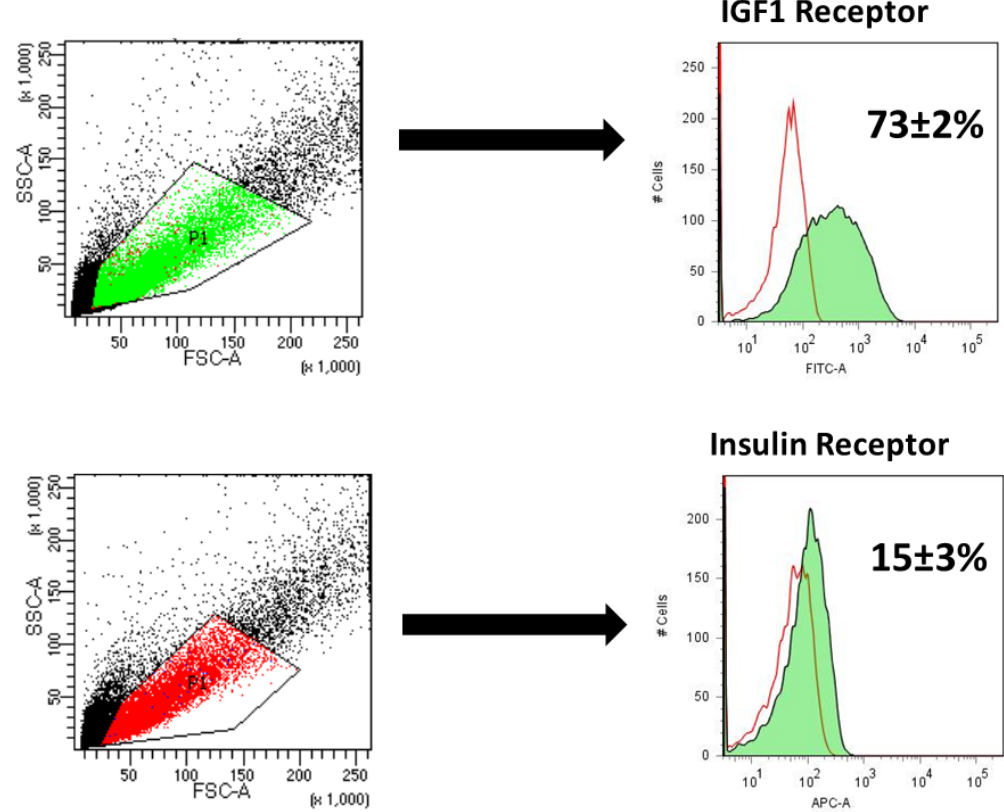

Figure 37. NTera2 cells express receptors for insulin-like signaling growth factors (A) RT-qPCR analysis of gene expression for insulin-like signaling-related genes. Graphs represent the results of two experiments carried out in duplicate and analyzed using the Mann-Whitney U test. (B) Flow cytometry analysis of the IGF1 receptor and insulin receptor in NTera2 cells. Graphs represent the results of two experiments. **p<0.01 


\section{Insulin-like growth factors phosphorylate the mitogenic signaling molecules AKT and}

MAP kinase and stimulate NTera2 proliferation in vitro in serum-free conditions

Insulin-like signaling is proposed to be important in TGCT pathogenesis, particularly through the actions of IGF1 and IGF1R [119]. The receptors IGF1R and INSR are activated by the peptides IGF1, IGF2, and insulin, and these activation signals are carried through mitogenic signaling pathways, of which the phosphorylation of the AKT and MAP kinase proteins are downstream events (Figure 4) [138]. Because I found NTera2 cells to express IGF1R and INSR at the mRNA and protein levels, with relatively low expression of $I G F 1$ and $I G F 2$, I investigated the functional response of these cells to stimulation by exogenous insulin-like growth factors. Western blot analysis of AKT and MAPK phosphorylation in NTera2 cells stimulated by IGF1, IGF2, or insulin, revealed that each of these growth factors was able to activate downstream mitogenic signaling pathways in these cells (Figure 38A). Importantly, supplementing NTera2 cells with these growth factors in serum-free conditions triggered a significant increase in their proliferation rate (Figure 38B). These results confirm that, like murine EC cells and TGCT tissues, NTera2 is functionally responsive to exogenous insulin-like signaling. Importantly, these results also suggest that autocrine insulin-like signaling is not an important aspect of NTera2 malignancy. 
A.

BSA IGF1 IGF2 INS

Phospho-AKT

Total AKT

Phospho-MAPK

Total MAPK

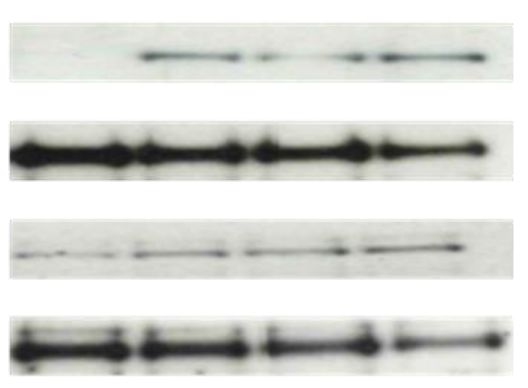

B.

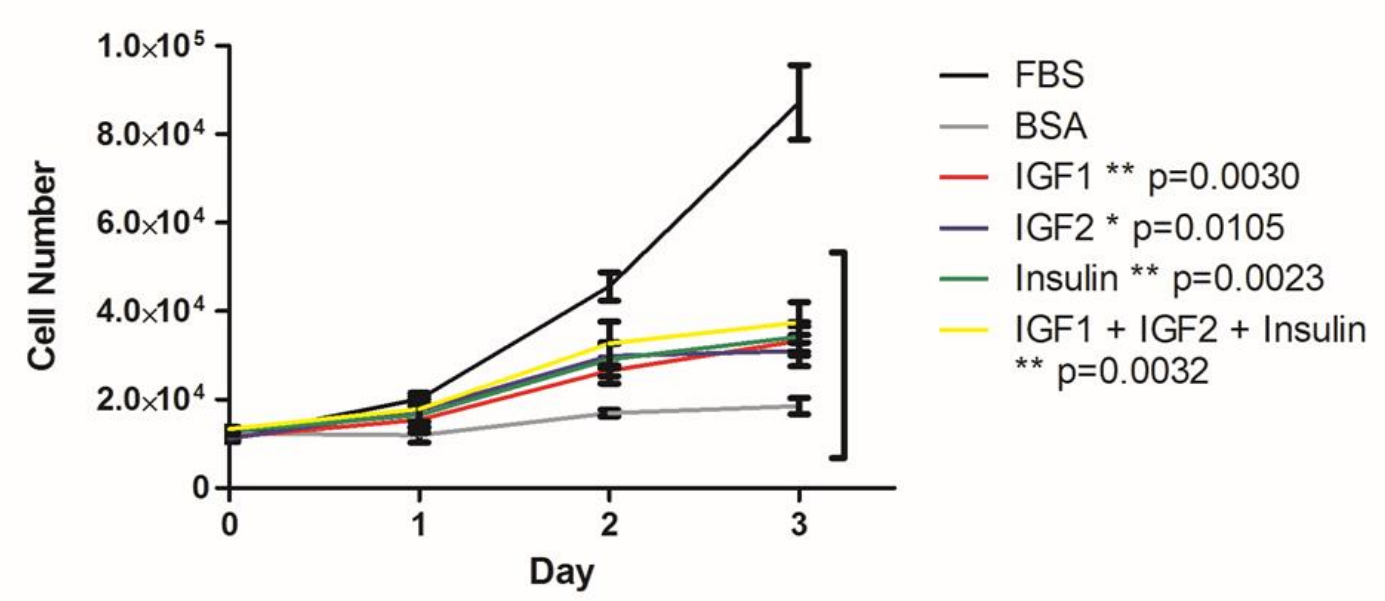

Figure 38. Insulin-like signaling growth factors stimulate NTera2 cells

(A) Western blot analysis of phosphorylated and total AKT and MAPK proteins

following stimulation of NTera2 cells with insulin-like signaling growth factors. The results of two independent experiments are represented by each blot. (B) Proliferation assay of NTera2 cells treated with insulin-like signaling growth factors. Graph represents the results of two experiments carried out in triplicate, where Day 6 cell numbers were compared to BSA and analyzed using a 2-tailed unpaired t-test. (BSA - bovine serum albumin; IGF1 - insulin-like growth factor I; IGF2 - insulin-like growth factor II; INS insulin; FBS - fetal bovine serum) $* \mathrm{p}<0.05, * * \mathrm{p}<0.01$ 


\section{DLK1 downregulation decreases NTera2 proliferation in vitro and NTera2 tumor size and organ seeding abilities in vivo}

Though previous studies have identified various roles of DLK1 in normal tissue growth and regulation, several studies have reported that DLK1 is important for the growth and stemness maintenance of certain cancers [10, 11, 32-34, 105, 139-141]. Indeed, downregulation of DLK1 in hepatocellular carcinoma cell lines was recently shown to decrease their in vivo tumor growth capabilities and increase their expression of differentiation markers [107]. Thus, after finding that the relatively high $D L K 1 / M E G 3$ expression ratio decreased in NTera2 cells during 5-azaD treatment in association with a decrease in the proliferation rate of these cells, I investigated the importance of $D L K 1$ in the proliferation rate of NTera2 cells. To accomplish this, I utilized two shRNAs against DLK1 and one control shRNA against a gene not expressed by human cells to create stably transfected cell lines. I confirmed the knockdown of $D L K 1$ in these cells (Figure 39A) and found that each transfected cell line retained similar distribution of DLK1 by immunohistochemistry (Figure 40). I assessed the in vitro proliferation rates of these cells and found that $D L K 1$ knockdown significantly decreased the NTera2 proliferation rate (Figure 39B). Interestingly, I also found that DLK1 knockdown was accompanied by a decreased in OCT4 expression, suggesting that these two genes are somehow linked.

Because the tumor microenvironment and normal serum levels of soluble DLK1 may influence TGCT tumor growth, I employed these stable transfected cells in vivo to study the tumorigenicity and organ seeding abilities of DLK1 knockdown cells. I found that knockdown of $D L K 1$ resulted in significantly decreased tumor growth (Figure 41A,B) and seeding efficiency of cells to the lung and liver (Figure 42B). Importantly, Figure 41C 
shows the survival curve for these mice. While this figure suggests that the control cells were somehow more lethal to the mice than DLK1 knockdown cells, the death of mice during this experiment occurred within the first 2.5 weeks and was likely due to the native immunodeficiency in SCID mice combined with their treatment with irradiation. Thus, these results collectively indicate that $D L K 1$ is important for the in vivo tumor growth and seeding efficiency of NTera2 cells. 
A.
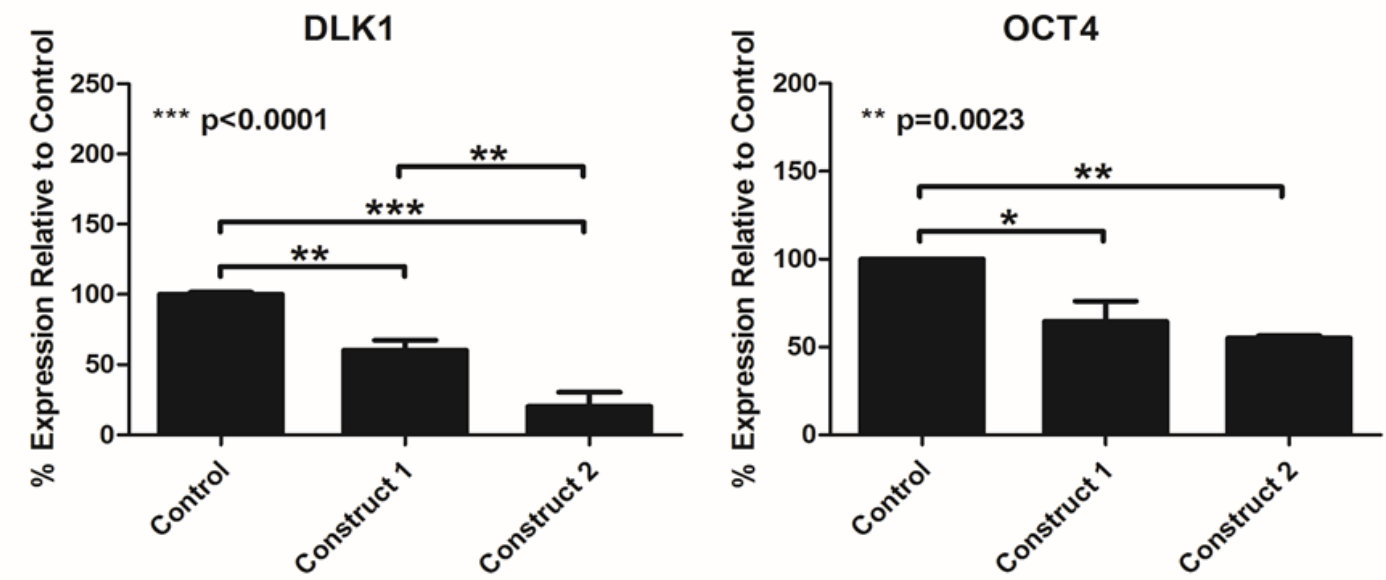

Percent Knockdown: $\quad 40 \% \quad 80 \%$

B.

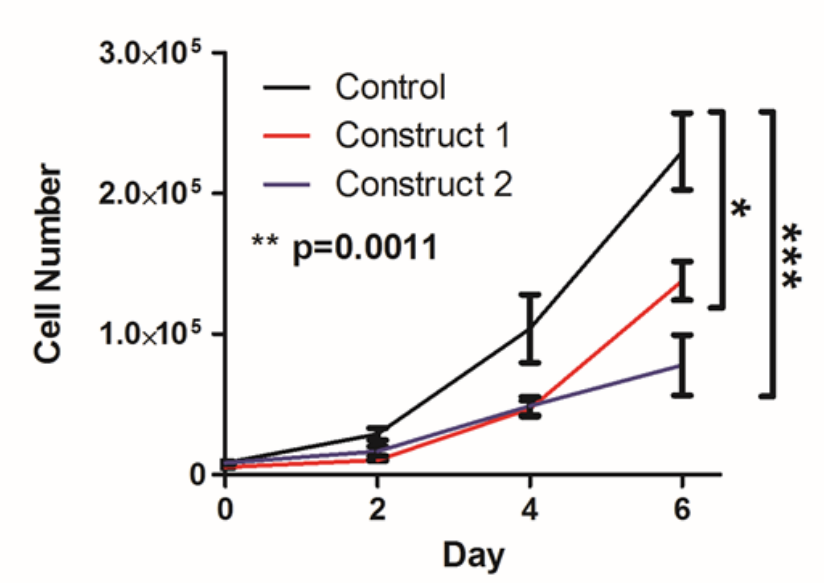

Figure 39. DLK1 knockdown decreases NTera2 OCT4 expression and in vitro cell proliferation

(A) RT-qPCR analyses of NTera2 cells stably transfected with either a control plasmid or a shRNA construct targeting $D L K 1$. Graphs represent the results of two experiments carried out in duplicate and analyzed using a one-way ANOVA with a Tukey post-test. (B) Proliferation assay of stably transfected NTera2 cells. Graph represents the results of two assays carried out in triplicate, where Day 6 cell numbers were analyzed using a one-way ANOVA with a Tukey post-test. *p $<0.05,{ }^{* *} \mathrm{p}<0.01, * * * \mathrm{p}<0.001$ 


\section{Control}

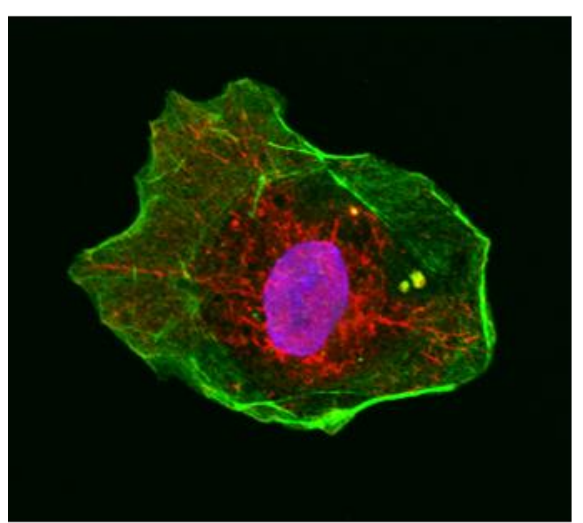

Construct 1

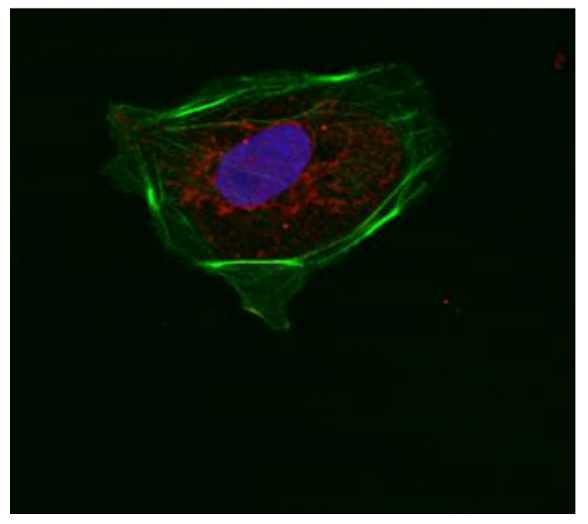

Construct 2

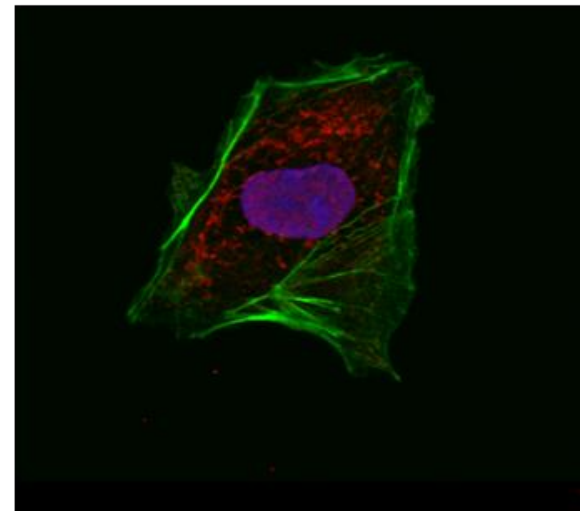

Figure 40. DLK1 knockdown cells exhibit strong nuclear DLK1 staining

Representative confocal 3D overlay of stably transfected NTera2 cells showing the nucleus (DAPI; blue), F-actin (green), and DLK1 (red). 
A.

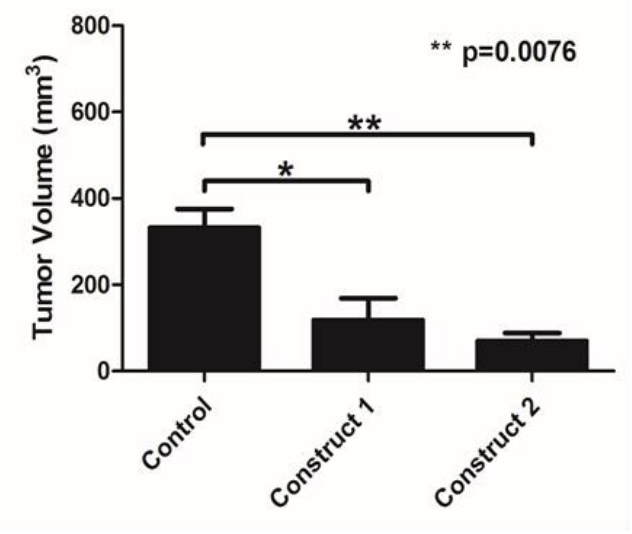

C.

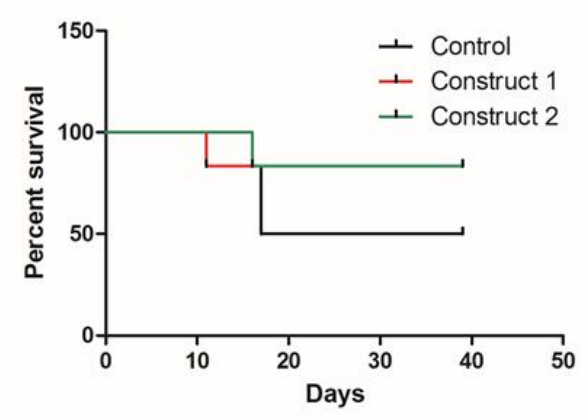

B. Control Construct 1 Construct 2

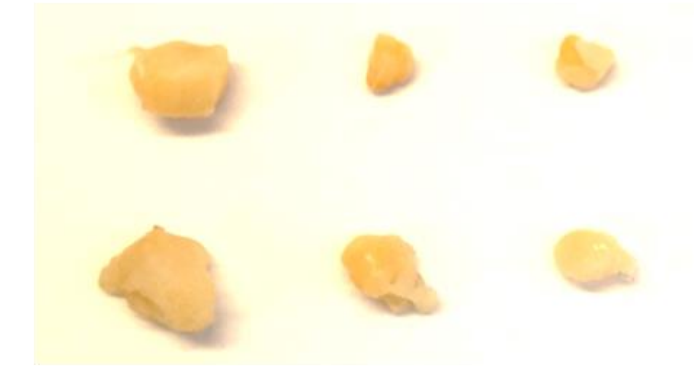

\section{Figure 41. DLK1 knockdown decreases NTera2 tumor growth in vivo}

(A) In vivo tumor volume in SCID mice at 5.5 weeks post-irradiation. Each mouse was injected with 8 million stably transfected NTera2 cells at 24 hours post-irradiation, and results were compared using a one-way ANOVA with a Tukey post-test. Graph represents the results of one experiment with Control $(n=3)$, Construct $1(n=5)$, and Construct $2(n=5)$. (B) Representative tumors for each of the stably transfected NTera2 cell lines. (C) Survival curve of animals injected with stably transfected NTera2 cells for the single experiment with $\mathrm{n}=6$ for each group at the beginning of the experiment $* \mathrm{p}<0.05 * * \mathrm{p}<0.01$ 
A.

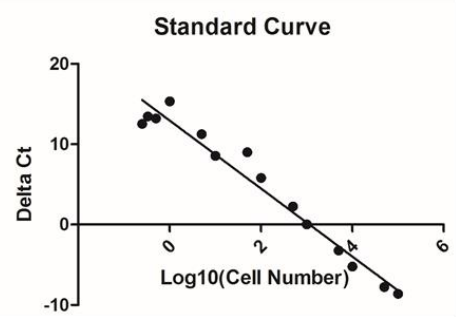

B.
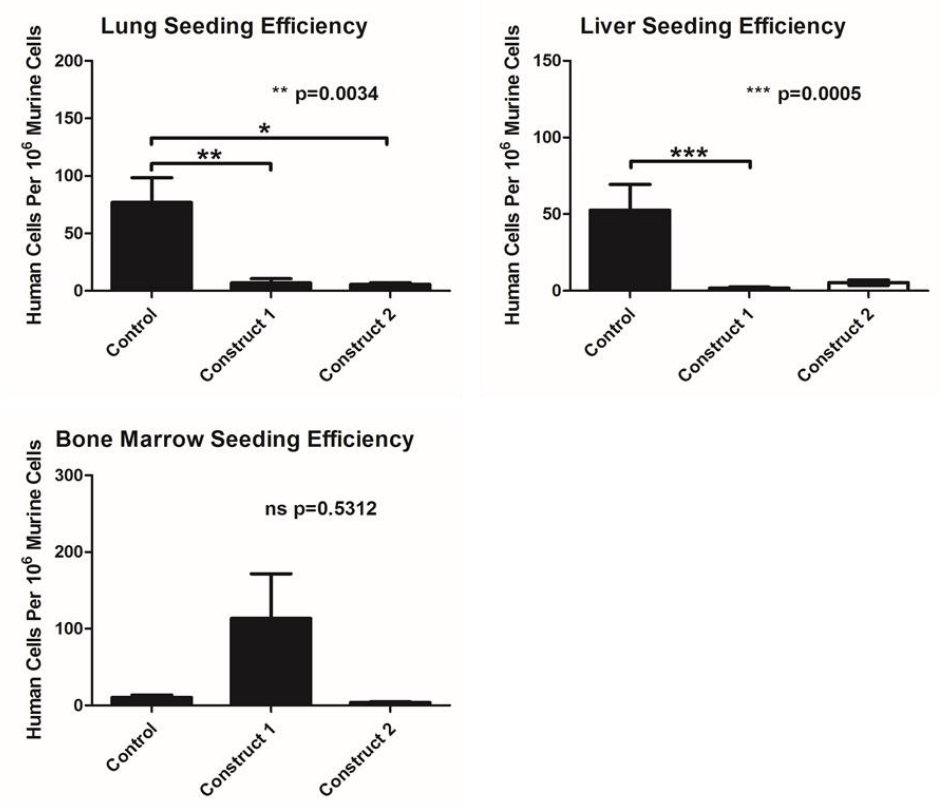

Figure 42. DLK1 knockdown decreases the in vivo organ seeding efficiencies of

\section{NTera2 cells}

(A) Standard curve generated based on qPCR analysis of DNA extracted from mixtures of NTera2 cells and murine bone marrow MNCs. (B) Seeding efficiencies of stably transfected NTera2 cells during in vivo tumor growth. Experiment was performed on organs harvested from the same animals from which tumors were harvested and measured. Graphs represent the results of one experiment with Control $(n=3)$, Construct $1(n=5)$, or Construct $2(\mathrm{n}=5)$. Analysis of DNA from each organ was performed using qPCR once in duplicate for lung and liver samples, and twice in duplicate for bone marrow samples, and results were analyzed using the Kruskal-Wallis H test with Dunn's post-test. ${ }^{*} \mathrm{p}<0.05$, $* * \mathrm{p}<0.01, * * * \mathrm{p}<0.001$ 
Embryonal carcinoma tissue samples exhibit PGC-like imprinting at the IGF2-H19 and DLK1-MEG3 loci

EC cells are considered the pluripotent-like cells of teratocarcinomas and are often found in mixed germ cell tumors [30,54]. As such, I investigated the IGF2-H19 ICR and DLK1-MEG3 IG-DMR imprints at multiple locations within mixed EC tissues (Figure 43). I found that the IGF2-H19 ICR displayed low methylation in these tissues, regardless of locality, in agreement with previous studies on GCTs [36-38]. Similarly, low methylation was also found at the DLK1-MEG3 IG-DMR in these tissues, and the methylation status of this DMR was also consistent between different sites of the same tissue. These results suggest that mixed EC tissues exhibit relatively homogeneous PGC-like methylation of the H19-IGF2 ICR and DLK1-MEG3 IG-DMR. However, considering that more stem-like cells like those which established the NTera2 cell line can be isolated from mixed GCTs such as teratocarcinomas, these imprinting patterns may represent only the bulk tumor tissue of low stemness, whereas more stem-like cells may be interspersed within these tissues which exhibit higher methylation at the DLK1-MEG3 IG-DMR. It is also worth noting that due to the hypermethylated P57-LIT1 locus I previously found in NTera2 cells, I also assayed that DMR in EC patient samples. Like the other two loci, P57-LIT1 was also hypomethylated in these tissues. 


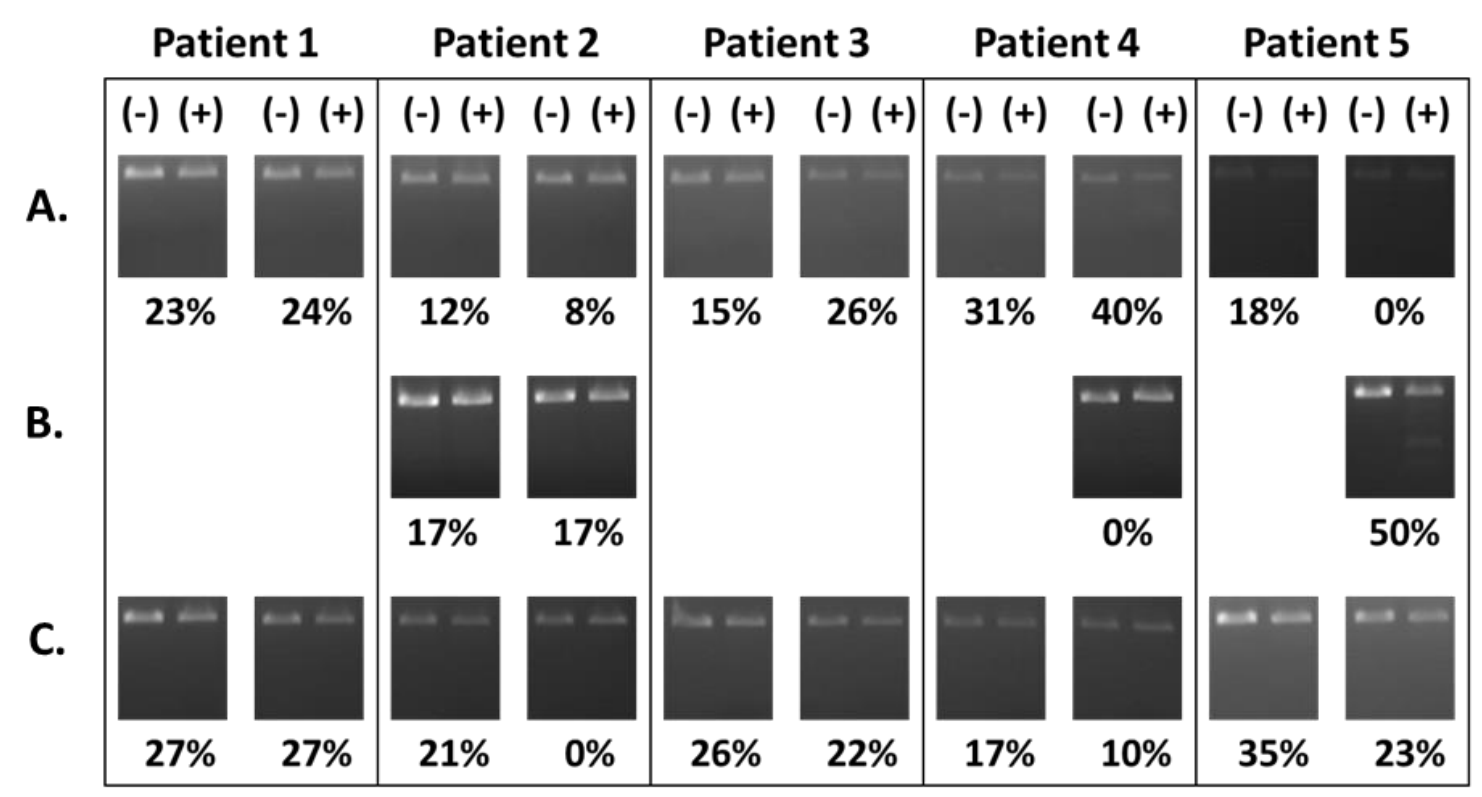

Figure 43. Human primary EC tissue exhibits hypomethylation at the IGF2-H19, DLK1-MEG3, and P57-LIT1 loci

COBRA of BSgDNA amplicons from the IGF2-H19 ICR (A), DLK1-MEG3 IG-DMR (B), and P57-LIT1 DMR of FFPE human EC tissues. Each graph represents the result of one experiment. 


\section{Cancer stem cell surface markers CD133 and SSEA4 mark distinct subpopulations of NTera2 with unique gene expression signatures of cell stemness}

The surface expression of CD133 and SSEA4 by EC cells and tissues has been previously reported $[81,142]$. Because these markers have been used to study CSC-like populations of cells in several tissues and cell lines, I employed them to identify potential CSC subpopulations of NTera2 cells $[67,143]$. In line with previous studies, I found that NTera2 cells express CD133 and SSEA4 on their surface, and I sorted 4 subpopulations of cells based on the expression of these markers (Figure 44) [80, 81]. Due to the importance of genes within the IGF2-H19 and DLK1-MEG3 loci in stem cell maintenance and proliferation, I assessed the expression of imprinted genes from these loci along with pluripotency-related genes in freshly sorted cells to identify potential differences in cell stemness (Figure 45B, 46; p values for these graphs located in Table 7) [5, 6]. Similarly, I assessed the expression of $D L K 1$ and $M Y C$ in unsorted NTera 2 cells and testes to confirm their upregulation in EC (Figure 45A).

I found that SSEA4 ${ }^{+}$cell subpopulations had the highest expression of the pluripotency-related TDGF1 gene, which encodes the CRIPTO1 protein previously shown to mark an especially tumorigenic subpopulation of NTera2 cells having high pluripotency gene expression [144]. This result also agrees with a recent report in which SSEA4 surface expression helped distinguish cells within EC tissue which had high pluripotency gene expression and could differentiate into cells from all three germ layers upon embryoid body formation [69]. In addition, I found that the CD133-SSEA4+ cells had significantly higher expression of the pluripotency gene $O C T 4$ and a higher $D L K 1 / M E G 3$ expression ratio, with a trend towards a higher IGF2/H19 expression ratio, than CD133-SSEA4 ${ }^{-}$cells, potentially 
indicating differences in the stemness and proliferation rate of these subpopulations. Interestingly, significant differences in the expression of $I G F 2$ and $H 19$ were found among the subpopulations, but no significant differences were found for $D L K 1$ or $M E G 3$. Finally, the lowest DLK1/MEG3 ratio was found in CD133-SSEA4 cells, along with a trend for the highest $H 19$ expression, suggesting that the CD133-SSEA4 phenotype may mark a particularly quiescent subpopulation of NTera2 cells. Overall, these results demonstrate that subpopulations of cells within the NTera2 cell line can be distinguished based on CD133 and SSEA4 surface expression, and these cell subpopulations carry unique expression signatures for imprinted and pluripotency-related genes. 

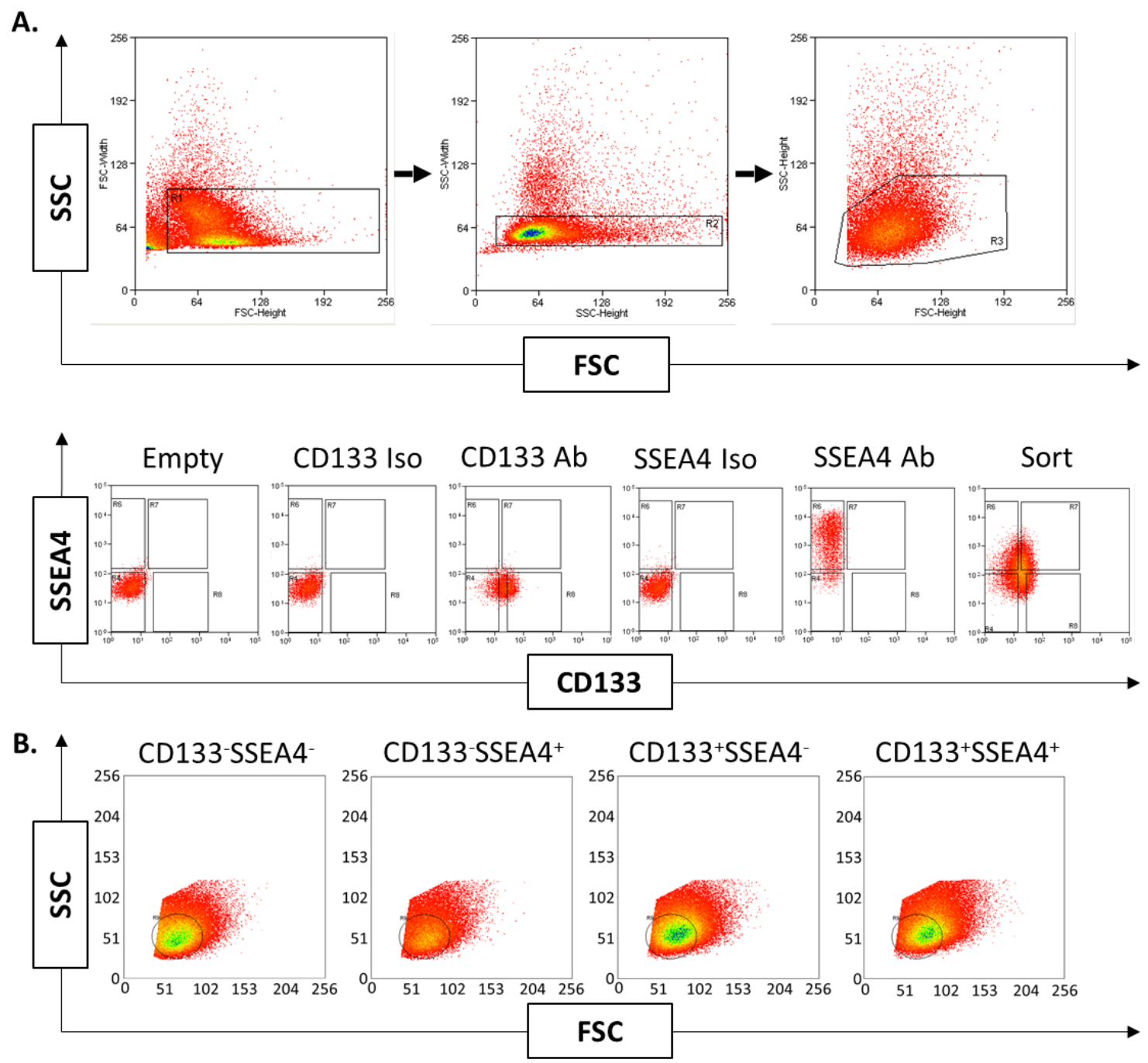

Figure 44. Sorting strategy for NTera2 subpopulations based on CSC markers CD133 and SSEA4

(A) Isolation of NTera2 cells based on CD133 and SSEA4 surface expression. The top graph represents the size exclusion parameters and the bottom graph represents the isotype controls used. (B) Cells isolated from each subpopulation were of the same size. 
A.

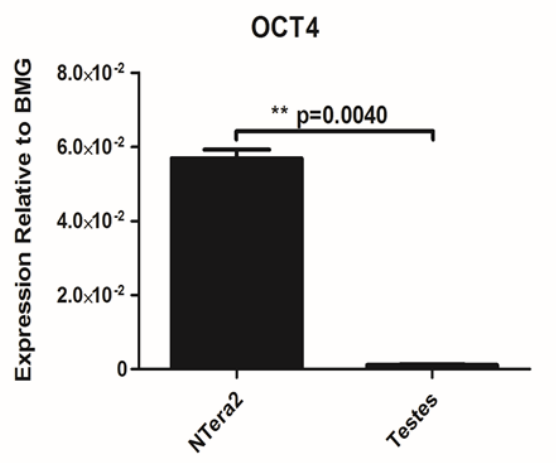

B.

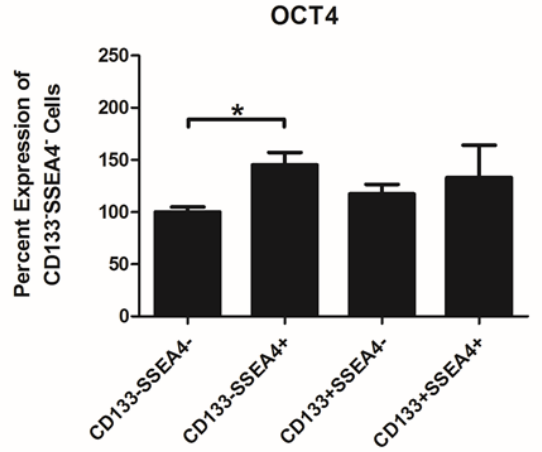

MYC

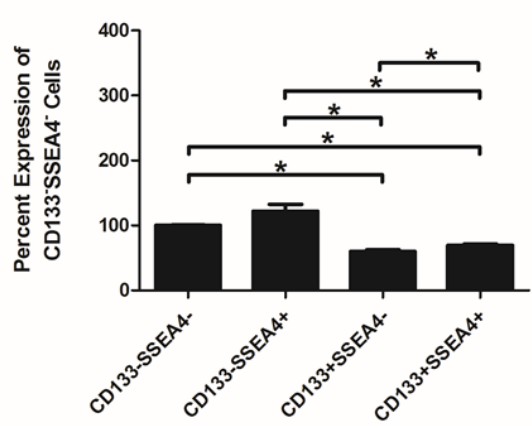

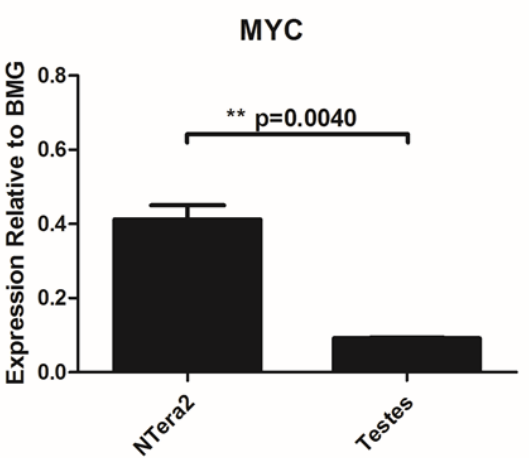

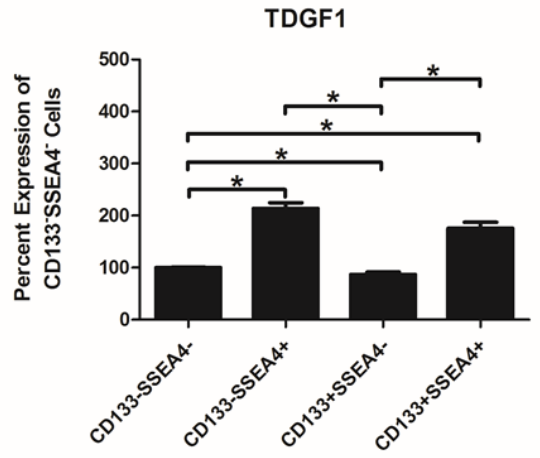

Figure 45. The CSC markers CD133 and SSEA4 distinguish subpopulations of

\section{NTera2 cells exhibiting unique gene expression signatures}

(A) RT-qPCR analysis of pluripotency gene expression. Graphs represent the results of two experiments carried out in duplicate and analyzed using the Mann-Whitney U test. (B) RT-qPCR analysis of pluripotency-related genes. Graphs represent the results of two experiments carried out in duplicate and analyzed using the Mann-Whitney U test. *p $<0.05$ $* * \mathrm{p}<0.01$ 

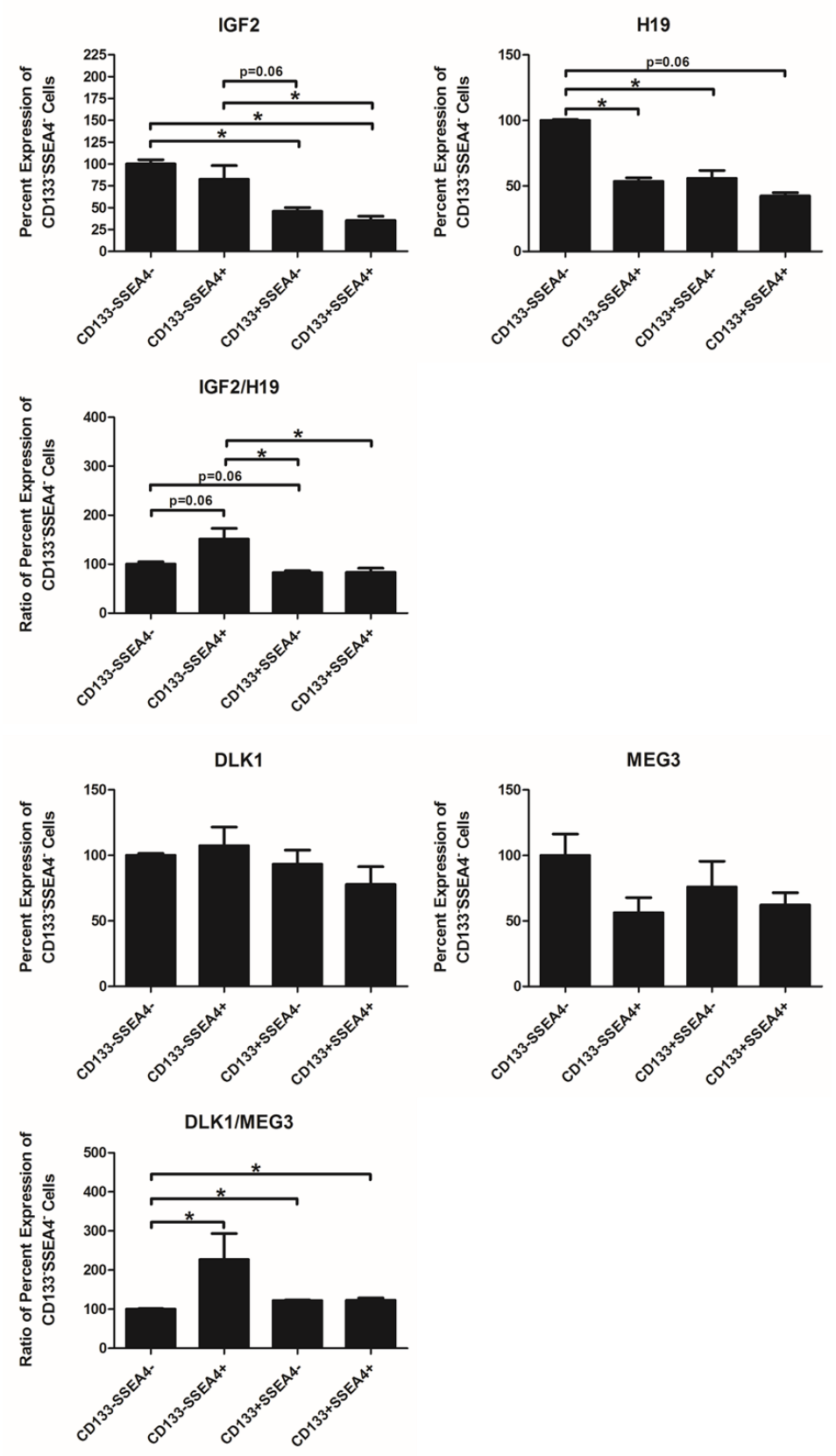

Figure 46. NTera2 subpopulations exhibit differences in their expression of imprinted genes

RT-qPCR analysis of genes from the IGF2-H19 and DLKI-MEG3 loci. Graphs represent the results of two experiments carried out in duplicate and analyzed using the MannWhitney U test. *p $<0.05$ 


\begin{tabular}{|c|c|c|c|c|c|c|c|c|c|}
\hline & \multicolumn{3}{|c|}{ OCT4 } & \multicolumn{3}{|c|}{ TDGF1 } & \multicolumn{3}{|c|}{ CMYC } \\
\hline & 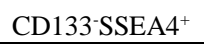 & CD133+SSEA4 & $\mathrm{CD} 133^{+} \mathrm{SSEA}^{+}$ & $\mathrm{CD}_{133} \mathrm{SSEA4}^{+}$ & CD133+SSEA4 & $\mathrm{CD}_{133^{+} \mathrm{SSEA4}^{+}}$ & 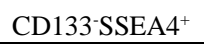 & CD133+SSEA4 & $\mathrm{CD}_{133^{+} \mathrm{SSEA}} 4^{+}$ \\
\hline CD133 SSEA4 & 0.0286 & 0.2000 & 1.0000 & 0.0286 & 0.0286 & 0.0286 & 0.1143 & 0.0286 & 0.0286 \\
\hline CD133-SSEA4 ${ }^{+}$ & & 0.2000 & 1.0000 & & 0.0286 & 0.1143 & & 0.0286 & 0.0286 \\
\hline CD133+'SSEA4 & & & 1.0000 & & & 0.0286 & & & 0.0286 \\
\hline
\end{tabular}

\begin{tabular}{|c|c|c|c|c|c|c|c|c|c|}
\hline & \multicolumn{3}{|c|}{ IGF2 } & \multicolumn{3}{|c|}{ H19 } & \multicolumn{3}{|c|}{ IGF2/H19 } \\
\hline & 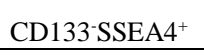 & CD133+'SSEA4 & $\mathrm{CD}_{133^{+}{ }^{+} \mathrm{SSEA} 4^{+}}$ & CD133-SSEA4 $^{+}$ & CD133+SSEA4 & $\mathrm{CD}_{133^{+}{ }^{+} \mathrm{SSEA}^{+}}$ & $\mathrm{CD} 133-\mathrm{SSEA}^{+}$ & CD133+'SSEA4- & $\mathrm{CD} 33^{+} \mathrm{SSEA}^{+}$ \\
\hline CD133'SSEA4 & \multirow[t]{3}{*}{0.6857} & 0.0286 & 0.0286 & \multirow[t]{3}{*}{0.0286} & \multirow{3}{*}{$\begin{array}{l}0.0286 \\
1.0000\end{array}$} & 0.0571 & \multirow[t]{3}{*}{0.0571} & 0.0571 & 0.1143 \\
\hline CD133-SSEA4 ${ }^{+}$ & & \multirow{2}{*}{0.0286} & 0.0571 & & & 0.0571 & & \multirow{2}{*}{0.0286} & 0.0286 \\
\hline \multirow[t]{4}{*}{$\mathrm{CD} 33^{+}{ }^{+} \mathrm{SSEA4} 4^{-}$} & & & 0.2000 & & & 0.1143 & & & 0.8857 \\
\hline & & & & & & & & & \\
\hline & \multicolumn{3}{|c|}{ DLK1 } & \multicolumn{3}{|c|}{ MEG3 } & \multicolumn{3}{|c|}{ DLK1/MEG3 } \\
\hline & 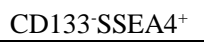 & $\mathrm{CD}^{2} 33^{+}$SSEA4 & $\mathrm{CD}_{133^{+} \mathrm{SSEA}^{+}}$ & CD133-SSEA4 $^{+}$ & CD133+'SSEA4 & $\mathrm{CD}_{133^{+}} \mathrm{SSEA4}^{+}$ & $\mathrm{CD}^{2} 33-\mathrm{SSEA4}^{+}$ & CD133+'SSEA4- & $\mathrm{CD} 33^{+} \mathrm{SSEA4}^{+}$ \\
\hline CD133-SSEA4- & \multirow[t]{3}{*}{1.0000} & 1.0000 & 0.4857 & \multirow[t]{3}{*}{0.1143} & 0.3429 & 0.1143 & \multirow[t]{3}{*}{0.0286} & \multirow{3}{*}{$\begin{array}{l}0.0286 \\
1.0000\end{array}$} & 0.0286 \\
\hline CD133-SSEA4 $^{+}$ & & 0.3429 & 0.3429 & & 0.8857 & 0.8857 & & & 0.8857 \\
\hline CD133+SSEA4- & & & 0.3429 & & & 0.8857 & & & 1.0000 \\
\hline
\end{tabular}

Table 7. Individual $\mathrm{p}$ values for Figures 45 and 46 


\section{NTera2 subpopulations sorted by CD133 and SSEA4 surface expression display unique migratory characteristics in vitro and in vivo}

Previous studies have found that the CD133 and SSEA4 antigens help identify CSC-like cells with increased migratory abilities $[67,145]$. To determine if NTera2 cells exhibit different migratory abilities based on CD133 and SSEA4 expression, I employed freshly sorted NTera2 subpopulations in chemotaxis assays towards a 10\% FBS gradient (Figure 47A; p values for Figure 47 listed in Table 8) and found statistically significant higher migration by the SSEA4 ${ }^{+}$cell subpopulations compared to CD133-SSEA4- cells.

To determine if the differences in in vitro migration by NTera2 subpopulations to $10 \%$ FBS corresponded to differences in in vivo organ seeding abilities, I injected freshly sorted cells from each NTera2 subpopulation intravenously into SCID mice and analyzed their organs for human-murine chimerism at 60 hours post-injection (Figure 47B). Interestingly, I found a significantly higher number of CD133-SSEA4 cells in the bone marrow than $\mathrm{CD} 133^{-} \mathrm{SSEA}^{+}{ }^{+}$or $\mathrm{CD} 133^{+}{ }^{+} \mathrm{SSEA} 4^{-}$cells. Also, similar to the differences in in vitro migration responses of these cell subpopulations, significantly higher numbers of

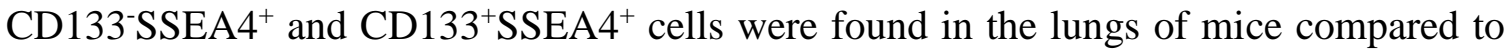
CD133-SSEA4 ${ }^{-}$cells. What's more, this preferential seeding of the lung was not due to the simple trapping of larger cells in lung capillaries, as cells of each subpopulation were of similar sizes (Figure 44B). Collectively, these results demonstrate that surface expression of the CD133 and SSEA4 antigens identifies unique NTera2 cell subpopulations which exhibit different in vitro migratory properties and in vivo organ seeding efficiencies. 
A.

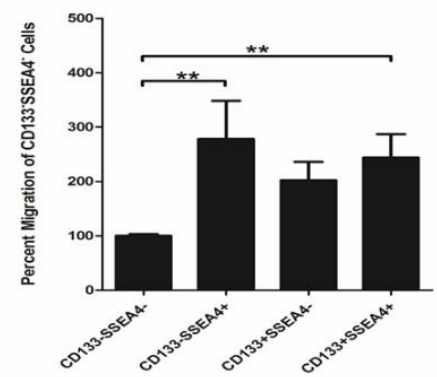

B.
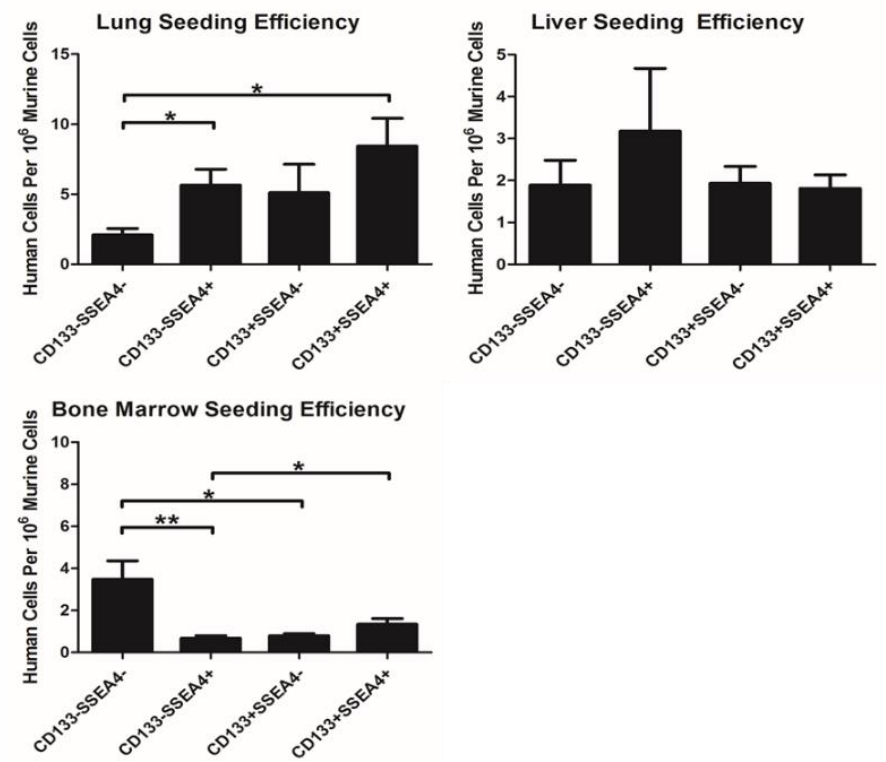

Figure 47. NTera2 subpopulations demonstrate unique migratory abilities in vitro and in vivo

(A) Chemotaxis of NTera2 subpopulations in vitro. Graph represents the ratio of migration towards FBS versus BSA (negative control) and calculated as a percent migration of CD133-SSEA4 cells. Chemotaxis of freshly sorted cells was carried out a total of three times in duplicate and results were analyzed using the Mann-Whitney U test. (B) Organ seeding efficiencies of freshly sorted NTera2 subpopulation cells upon sacrifice of SCID mice at 60 hours post-intravenous injection. Results for each group ( $\mathrm{n}=4$; one experiment total) were assayed by qPCR at least twice in duplicate and analyzed using the MannWhitney U test. $* \mathrm{p}<0.05, * * \mathrm{p}<0.01$ 


\begin{tabular}{|c|c|c|c|}
\hline & \multicolumn{3}{|c|}{ Chemotaxis } \\
\hline & CD133-SSEA4 ${ }^{+}$ & CD133+SSEA4 & $\mathrm{CD} 133^{+} \mathrm{SSEA} 4^{+}$ \\
\hline CD133-SSEA4 & 0.0043 & 0.1797 & 0.0043 \\
\hline CD133-SSEA4 $^{+}$ & & 0.8182 & 0.9372 \\
\hline CD133+SSEA4 & & & 0.8182 \\
\hline
\end{tabular}

\begin{tabular}{|c|c|c|c|c|c|c|c|c|c|}
\hline & \multicolumn{3}{|c|}{ Lung Seeding Efficiency } & \multicolumn{3}{|c|}{ Liver Seeding Efficiency } & \multicolumn{3}{|c|}{ Bone Marrow Seeding Efficiency } \\
\hline & CD133 SSEA4 $^{+}$ & CD133+SSEA4 $^{-}$ & CD133 $^{+}$SSEA4 $^{+}$ & CD133-SSEA4 $^{+}$ & CD133 $^{+}$SSEA4 ${ }^{-}$ & 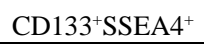 & CD133 SSEA4 $^{+}$ & CD133 $^{+}$SSEA4 & $\mathrm{CD}_{133^{+} \mathrm{SSEA}^{+}}{ }^{+}$ \\
\hline CD133-SSEA4 & 0.0426 & 0.8518 & 0.0293 & 0.7312 & 0.2790 & 0.3469 & 0.0014 & 0.0190 & 0.1095 \\
\hline${\mathrm{CD} 133-\mathrm{SSEA}^{+}}^{+}$ & & 0.3282 & 0.3282 & & 0.2475 & 0.1740 & & 0.2155 & 0.0334 \\
\hline CD133 $^{+}$SSEA4 ${ }^{-}$ & & & 0.1605 & & & 0.9525 & & & 0.1824 \\
\hline
\end{tabular}

三

Table 8. Individual p values for Figure 47 


\section{Each NTera2 subpopulation sorted by CD133 and SSEA4 surface expression re- establishes the CD133- and SSEA4-expressing phenotypes of the parental NTera2 cell line upon in vitro expansion}

Several recent studies have demonstrated that surface marker expression is not a static feature of normal stem cells or cancerous stem-like cells [70, 72-74]. Indeed, primary leukemia cells and low-passage metastatic melanoma cancer cell lines were recently found to exhibit fluctuations in their surface expression of CSC markers [70, 71]. Notably, single cells sorted from the established human ovarian carcinoma cell line A2780 based on CD24 and CD44 surface expression could regain expression of either CD24, CD44, or both antigens upon in vitro expansion [74]. Considering this discovery, I sorted single cells from the NTera2 cell line based on CD133 and SSEA4 surface expression, and I re-analyzed the expression of these antigens following in vitro expansion of these cells (Figure 48). I found that expansion of single cells from each NTera2 subpopulation (CD133-SSEA4', CD133$\mathrm{SSEA}^{+}, \mathrm{CD} 133^{+} \mathrm{SSEA}^{-}, \mathrm{CD} 133^{+} \mathrm{SSEA}^{+}$) resulted in re-establishment of the parental cell CD133 and SSEA4 surface expression phenotypes. Thus, the surface expression of CSC markers CD133 and SSEA4 by NTera2 cells fluctuates over time, suggesting that the true stemness of individual NTera2 cells cannot be assessed directly by the surface expression of these antigens. 


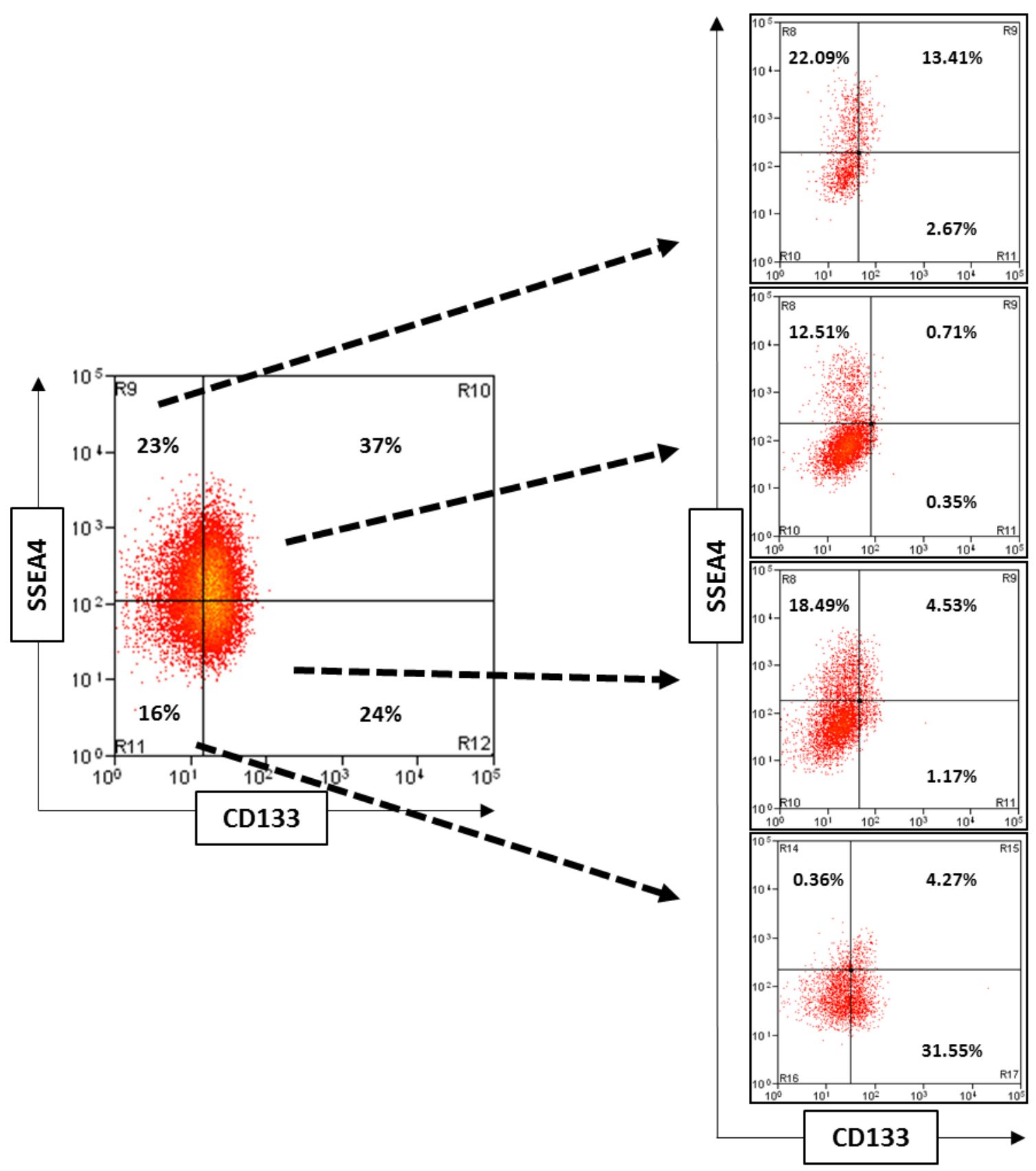

Figure 48. Singly sorted NTera2 cells exhibit a fluctuating phenotype upon in vitro expansion

Staining of in vitro expanded single cells following FACS sorting based on CD133 and SSEA4 expression. 


\section{Discussion}

Herein I provide evidence that the paternally imprinted growth-related gene $D L K 1$ is important for the malignancy of the human EC cell line NTera2. Whereas the paternally imprinted IGF2-H19 locus has been thoroughly investigated in TGCTs, where a hypomethylated ICR often remains as an artifact of the proposed PGC origin of these tumors, no study has directly investigated imprinting at the $D L K 1-M E G 3$ locus in these tissues [36-38, 122]. What's more, a recent investigation into the imprinting status of PGCs during their transformation into EGCs, and thus their acquisition of pluripotency and teratoma-forming capacity, did not examine the DLK1-MEG3 locus [146]. This omission is especially surprising given the seminal work by Kono, et al., which demonstrated the reliance of bimaternal mouse embryo growth and development specifically on the imprinting status of the IGF2-H19 and DLK1-MEG3 loci - two of only four known paternally imprinted loci in mice [15-17]. Moreover, the induction of pluripotency in murine PGCs by $M y c$ overexpression, a pluripotency-related gene which I found elevated in NTera2 cells (Figure 45A), upregulated $D l k 1$ expression in these cells [147]. Thus, given the imprinting dynamics of developing PGCs and their malignant and non-malignant routes of pluripotency acquisition (Figure 5A,B), combined with the presence of a historically growth-restricting hypomethylated $I G F 2-H 19$ ICR in both quiescent pluripotent adult stem cells and TGCT tissues, investigations into these two paternally imprinted loci could help uncover some important details regarding stem cell quiescence and the development of malignant stem-like cells $[8,36-38,41]$.

I show that the NTera2 cell line exhibited hypomethylation at the IGF2-H19 ICR and hypermethylation at the DLK1-MEG3 IG-DMR and MEG3 DMR, in contrast to the 
somatic methylation of these DMRs in human MNCs and the recently published somatic methylation results for other human tissues including testes [124]. The low IGF2/H19 and high DLK1/MEG3 expression ratios in NTera2 cells, as compared with MNCs and testes, agree with their respective methylation values at these DMRs. Surprisingly, while NTera2 cells expressed lower IGF2 and MEG3 than testes, and there was no difference in $H 19$ expression between NTera2 cells and testes, NTera2 cells also expressed significantly lower $D L K 1$ than testes. However, this difference in $D L K 1$ expression is likely due to the presence of multiple cell types in human testes RNA, of which leydig cells are known to express high levels of DLK1 protein [148]. Importantly, DLK1 mRNA expression also does not provide insight into protein locality, and immunohistochemistry revealed a strong presence of DLK1 in the nucleus of NTera2 cells. This is a surprising observation given the limited commentary on DLK1 as a nuclear protein [127]. DLK1 is typically studied in its membrane-bound or cleaved, soluble form, and a study on mouse leydig cells showed very little, if any, nuclear DLK1 in these cells. Importantly, these differences in genomic imprinting and expression of imprinted genes among NTera2, MNCs, and testes suggest that the high $D L K 1 / M E G 3$ expression ratio in NTera2 cells may be important for their malignancy, and this could serve as a viable target for therapeutic intervention for EC.

The methyltransferase inhibitor 5-azaD was recently found to be particularly toxic to EC cells [52]. Interestingly, 5-azaD specifically targeted stem-like cells and decreased the pluripotency of EC cells while globally demethylating their DNA $[52,53]$. What's more, the 5-azaD analog 5-azaC was also recently found to decrease the proliferation of rhabdomyosarcoma cells while demethylating their IGF2-H19 ICR and decreasing their IGF2/H19 expression ratio [131]. In agreement with these studies, I show that nanomolar 
doses of 5-azaD decreased the proliferation rate of NTera2 cells and demethylated the MEG3 promotor, although this treatment did not affect methylation at the DLK1-MEG3 IG-DMR. 5-azaD treatment also increased the IGF2/H19 expression ratio and decreased the $D L K 1 / M E G 3$ expression ratio, likely through a combination of possible imprintingdisrupting mechanisms, including promotor demethylation at the IGF2 and MEG3 genes and the blocking of CTCF binding via covalent attachment of DNA methyltransferases to DNA [132]. Thus, changes in the IGF2/H19 and DLK1/MEG3 expression ratios accompanied a decrease in the proliferation rate of NTera2 cells resulting from 5 -azaD treatment, suggesting that these expression ratios are involved in NTera2 cell proliferation. Specifically, these results suggest that the DLK1/MEG3 expression ratio could be particularly important for the proliferation of NTera2 cells, as it decreased in response to 5-azaD treatment, whereas the IGF2/H19 expression ratio increased. Notably, the $I G F 2$ expression increased in treated cells without a significant change in $I G F 1 R$ expression, questioning the importance of insulin-like signaling in these cells. What's more, NTera2 cells express higher IGF1R and INSR than testes tissues and express these genes at the protein level, suggesting that 5-azaD cells should respond to higher IGF2 expression if they have functional receptors.

Insulin-like signaling is an important component of PGC migration and possibly TGCT pathogenesis [119]. Studies have shown that insulin-like signaling is important for teratocarcinoma cells, though no studies have investigated the response of human EC cells to insulin-like signaling growth factors [135-137]. In addition to being a source of DLK1 for the testes, leydig cells also secrete other hormones, including IGF1 and testosterone [148-150]. This indicates that, like many other cancers, exogenous growth factors in serum 
are likely a major source of growth stimulation for TGCTs such as EC. More importantly, this suggests that exogenous growth factors in the serum, such as IGF1, may compensate for low autocrine growth factor stimulation. In addition, NTera2 cells also express other growth factors which also stimulate mitogenic signaling pathways[151]. I show that, in spite of the inability of increased IGF2 expression by NTera2 cells to rescue their proliferation rate upon 5-azaD treatment, exogenous insulin-like signaling growth factors including IGF2 are able to stimulate mitogenic signaling pathways in these cells. In addition, while NTera2 cells exhibit a slow proliferation rate in the absence of serum, supplementing serum-free media with insulin-like signaling growth factors enhances the proliferation of NTera2 cells. Thus, NTera2 cells have functional insulin-like signaling receptors which likely contributes to their malignancy, though it is unlikely that autocrine IGF2 signaling plays a significant role in this process.

Unlike IGF2, the DLK1 protein is known to play roles in proliferation as both a membrane-bound and a soluble protein $[152,153]$. Thus, assessing a role for this protein in the proliferation of NTera2 cells was achieved through shRNA-mediated downregulation. I show that knockdown of $D L K 1$ in stably transfected NTera2 cell lines significantly decreased the proliferation rates of these cells in vitro. Most importantly, these in vitro results translated into significantly smaller tumor sizes in vivo in immunodeficient mice, as well as significantly reduced seeding efficiencies to the lungs and livers of these animals. These results clearly display a requirement of $D L K 1$ expression by NTera 2 cells for their malignancy, highlighting DLK1 as a potential target for therapy in EC. Interestingly, while the mechanisms by which DLK1 influence cell proliferation are still largely unknown and are often unique to a given cell type, DLK1 is a known marker of 
certain normal stem cells and CSC populations $[10,11,34,154]$. Considering that EC is regarded as the stem cell within teratocarcinomas, and that EC cells exhibit certain aspects of pluripotency, DLK1 may represent a novel biomarker for EC as a product of aberrant genomic imprinting in developing PGCs during their transition to a pluripotent stem-like state $[30,39,50,69]$.

Considering the heterogeneity of mixed GCTs and the rarity of CSCs in other types of tumor tissues, coupled with the CSC-like nature of the NTera2 cell line and its dependence on $D L K 1$ for malignancy, I investigated the DLK1-MEG3 IG-DMR methylation in primary human TGCT samples which contained varying components of EC $[65,155]$. I show that, in comparison to MNCs and published data for human testes, this DMR is hypomethylated in mixed TGCTs [124]. What's more, analysis of the IGF2-H19 ICR in these tissues also revealed hypomethylation, in agreement with previous reports on GCTs, and this characteristic was consistent among different regions of the same tumor [36-38]. These results are consistent with the proposed PGC origin of TGCTs, and they suggest that more stem-like cells, like those which clonally expanded to form the NTera2 cell line, may be found as small populations of CSCs harboring higher methylation at the DLK1-MEG3 locus within mixed TGCT tissues [43].

The existence of CSCs within tumor tissues and established cell lines has been the subject of much investigation and debate $[156,157]$. Recent reports offer a new perspective on this phenomenon by providing evidence that, like previous studies in hematopoietic stem cells and Chinese hamster ovary cells, the expression of putative CSC markers fluctuate over time [70-74]. Surprisingly, the isolation of potential CSC subpopulations from the ovarian carcinoma cell line A2780 based on CD24 and CD44 surface expression 
revealed that, although these subpopulations were unique in their organ seeding efficiencies in vivo, in vitro expansion of single cells from each subpopulation resulted in the gain of CD24, CD44, or both markers. Collectively, these studies indicate that the expression of stem cell-associated markers is not static in adult stem cells, CSCs, or established cell lines. I show here that a similar phenomenon occurs in NTera2 cells, whereby subpopulations of cells isolated from this cell line exhibit unique phenotypes upon isolation, but expansion of single cells from each subpopulation re-establishes the parental cell line phenotype at the level of surface antigen expression. Specifically, subpopulations of NTera2 cells isolated by FACS based on their surface expression of CD133 and SSEA4, antigens known to be expressed on normal stem cells as well as potential CSC populations, displayed unique expression signatures and migration characteristics in vitro and in vivo. Importantly, little difference in the pluripotency gene OCT4 was found among the subpopulations. Similarly, no significant differences in $D L K 1$ expression were observed either, although the DLK1/MEG3 expression ratio was lowest in the CD133-SSEA4- cells. In this regard, in vitro expansion of single cells isolated from each subpopulation demonstrated the abilities of $\mathrm{CD}^{2} 3^{-}{ }^{-} \mathrm{SSEA}^{-}$, and CD133-SSEA4- cells to produce $\mathrm{CD}_{133}{ }^{+}$SSEA4 ${ }^{+}$cells, indicating that the overall stemness of cells within each subpopulation is similar.

In summary, the quiescence-associated hypomethylation of the IGF2-H19 locus is surprisingly found in NTera2 cells as well as EC-containing mixed TGCT tissues. However, while these tissues also exhibit hypomethylation at the DLK1-MEG3 IG-DMR, this DMR and the MEG3 DMR are hypermethylated in NTera2 cells. Investigation into the growth-related genes from these paternally imprinted loci revealed that autocrine IGF2 
expression is not likely to be significant for the survival or proliferation of these cells. In contrast, $D L K 1$ expression maintenance is necessary for the malignancy of NTera2 in vitro as well as in vivo. Importantly, isolation of potential CSCs from the NTera2 cell line revealed little difference in the $O C T 4$ or $D L K 1$ expression among these cells, and revealed no difference in their stem-like capabilies. These results highlight DLK1 as a potential target for the treatment of EC and potentially for other TGCTs, as it represents a novel candidate biomarker for stem-like cells within these tissues.

\section{Conclusions}

- As expected for tumor cells proposed to arise from PGCs, NTera2 cells exhibit hypomethylation at the IGF2-H19 DMR. However, in contrast to PGCs, NTera2 cells exhibit hypermethylation at the DLK1-MEG3 DMR.

- Undifferentiated NTera2 cells exhibit differences in imprinting methylation at control DMRs within the IGF2-H19 and DLK1-MEG3 loci compared to tissues isolated from primary patient samples, which is likely due to the heterogeneity of these tissues and more differentiated components.

- $D L K 1$, as a product of the DLK1-MEG3 locus, appears to be a crucial factor for the in vitro and in vivo growth of NTera2 and represents a potentially novel therapeutic target for EC. 
- Confirmation of NTera2 proliferation inhibition by the DNA demethylating compound 5-azadeoxycytidine is accompanied by demethylation of the MEG3 promotor and a decrease in the DLK1/MEG3 transcription ratio.

- The roles of noncoding RNAs from the DLK1-MEG3 and IGF2-H19 loci in the pathogenesis of embryonal carcinoma requires further studies. However, NTera2 cells express IGF1R and INSR and respond to insulin-like growth factor treatment.

- I cannot identify a static phenotype for potential subpopulation of CSCs within the established NTera2 cell line, supporting the idea that cells from established cell lines show a fluctuating phenotype for the expression of CSC markers. 


\section{CHAPTER 5: CONCLUSIONS AND FUTURE DIRECTIONS}

As studies continue to document aberrant imprinting and expression from the DLK1-MEG3 locus in various diseases, its hidden complexities are slowly unwinding to reveal a deep connection with the stem-like state of cells. The prospective origins of AML and EC in the PGC compartment underscores the potential importance of genomic imprinting in the development of these diseases. My results indicate that the expression of the imprinted $D L K 1$ gene is important for the malignancy of these cells, and this is likely due in part to its abilities to maintain cell stemness. In this context, the presence of nuclear DLK1 in NTera2 cells suggests a role for this protein in regulating gene transcription, and warrants investigation into DLK1 locality in AML blasts. Interestingly, NTera2 cells did not have higher $D L K 1$ expression than testes tissue, further highlighting the importance of DLK1 location when considering its roles in malignancy. However, the malignant transformation of ESCs towards an EC-like phenotype as a result of prolonged in vitro passaging is accompanied by an increase in $D L K 1$ expression, so monitoring the DLK1 localization during this process could also help determine its roles in malignancy [31]. Future studies on the activities of nuclear DLK1 will give valuable insight into its oncogenic functions, and may uncover treatment options for cancers which do not appear to involve DLK1 through simple RT-qPCR screening. Similarly, the balance between normal stem cell self-renewal and oncogenesis may be rooted in DLK1 activities.

One of the major hurdles in regenerative medicine is the potential for malignant transformation by pluripotent stem cells. Billions of dollars have been spent over the last 
two decades in an effort to safely harness the regenerative capacity of embryonic stem cells [158]. However, the underlying safety concern for teratoma formation by ESCs and iPSCs has been among the most significant roadblocks to their appearance in the clinic [159]. In this regard, investigations into adult pluripotent stem cell populations have yielded several potentially overlapping populations of cells which meet many of the criteria for pluripotency. Among these lies VSELs, which arguably lie near the top of the adult stem cell hierarchy due to their repertoire of stem cell and epiblast markers and their epigenetic signature $[44,160]$. Importantly, hypomethylation of the IGF2-H19 ICR is a characteristic shared by VSELs, migrating PGCs, and GCTs [8, 36-38, 41]. The potential feedback loop which exists between the OCT4 protein, the H19 RNA, and the IGF2-H19 imprint offers a unique perspective on the self-renewal of these cells, and a recent study investigating the downregulation of $H 19$ in EC cells discovered that $H 19$ knockdown cells had significantly decreased expression of several pluripotency markers, including OCT4 [18, 161]. Thus, while this imprinting pattern in VSELs and migrating PGCs is associated with their quiescence, the same imprinting pattern is associated with pluripotency maintenance and malignancy in EC and potentially other GCTs [43]. What's more, in contrast to the PGClike imprint at the IGF2-H19 locus, murine VSELs harbor a somatic imprint at the DLKIMEG3 IG-DMR [8]. Similarly, I found that the IG-DMR and MEG3 DMR are both hypermethylated in EC cells. These similarities in the imprinting patterns of VSELs and NTera2, which are both proposed descendants of PGCs, combined with the requirement for $D L K 1$ by NTera2 for proliferation, begs the question of what role the DLK1 protein plays in the proliferation of VSELs. 
Incredibly, over a decade of VSELs research has recently culminated in the successful expansion of these cells via treatment with the pan-histone deacetylase inhibitor (HDACi) valproic acid (VPA) [9]. This global epigenetic modifier was found to upregulate the expression of $D L K 1$ in human umbilical cord blood hematopoietic stem cells in addition to promoting their expansion ex vivo [48]. The nuclear localization of DLK1 in NTera2 cells is very surprising, especially considering that OCT4 expression was significantly higher in NTera2 cells than testes and was significantly decreased in DLK1 knockdown cells. Given the association of DLK1 with the stemness maintenance and proliferation of normal and cancerous stem cells, the intranuclear transport and activity of this protein may serve underappreciated functions specifically for stem cells, and investigations into genomic imprinting at the DLK1-MEG3 locus and DLK1 localization in VSELs could provide greater insight into the self-renewing potential of these cells. 


\section{REFERENCES}

1. Keating, S.T. and A. El-Osta, Epigenetics and metabolism. Circ Res, 2015. 116(4): p. 71536.

2. Ryall, J.G., et al., The NAD(+)-dependent SIRT1 deacetylase translates a metabolic switch into regulatory epigenetics in skeletal muscle stem cells. Cell Stem Cell, 2015. 16(2): p. 171-83.

3. Zhao, Y.T., M. Fasolino, and Z. Zhou, Locus- and cell type-specific epigenetic switching during cellular differentiation in mammals. Front Biol (Beijing), 2016. 11(4): p. 311-322.

4. Stadtfeld, M., et al., Aberrant silencing of imprinted genes on chromosome 12qF1 in mouse induced pluripotent stem cells. Nature, 2010. 465(7295): p. 175-81.

5. Venkatraman, A., et al., Maternal imprinting at the H19-Igf2 locus maintains adult haematopoietic stem cell quiescence. Nature, 2013. 500(7462): p. 345-9.

6. Qian, P., et al., The DIk1-Gt/2 Locus Preserves LT-HSC Function by Inhibiting the PI3KmTOR Pathway to Restrict Mitochondrial Metabolism. Cell Stem Cell, 2016. 18(2): p. 214-28.

7. Zhou, Y., et al., Activation of paternally expressed genes and perinatal death caused by deletion of the Gt/2 gene. Development, 2010. 137(16): p. 2643-52.

8. Shin, D.M., et al., Novel epigenetic mechanisms that control pluripotency and quiescence of adult bone marrow-derived Oct4(+) very small embryonic-like stem cells. Leukemia, 2009. 23(11): p. 2042-51.

9. Ratajczak, M.Z., et al., A Novel View of the Adult Stem Cell Compartment From the Perspective of a Quiescent Population of Very Small Embryonic-Like Stem Cells. Circ Res, 2017. 120(1): p. 166-178.

10. Begum, A., et al., DLK1, delta-like 1 homolog (Drosophila), regulates tumor cell differentiation in vivo. Cancer Lett, 2012. 318(1): p. 26-33.

11. $\mathrm{Xu}, \mathrm{X}$., et al., DLK1 as a potential target against cancer stem/progenitor cells of hepatocellular carcinoma. Mol Cancer Ther, 2012. 11(3): p. 629-38.

12. Schneider, G., et al., The paternally imprinted DLK1-GTL2 locus is differentially methylated in embryonal and alveolar rhabdomyosarcomas. Int J Oncol, 2014. 44(1): p. 295-300.

13. Takai, D., et al., Large scale mapping of methylcytosines in CTCF-binding sites in the human $\mathrm{H} 19$ promoter and aberrant hypomethylation in human bladder cancer. Hum Mol Genet, 2001. 10(23): p. 2619-26.

14. Han, L., D.H. Lee, and P.E. Szabo, CTCF is the master organizer of domain-wide allelespecific chromatin at the H19/lgf2 imprinted region. Mol Cell Biol, 2008. 28(3): p. 112435.

15. Kono, T., et al., Birth of parthenogenetic mice that can develop to adulthood. Nature, 2004. 428(6985): p. 860-4.

16. Bartolomei, M.S. and A.C. Ferguson-Smith, Mammalian genomic imprinting. Cold Spring Harb Perspect Biol, 2011. 3(7). 
17. Kawahara, M., et al., High-frequency generation of viable mice from engineered bimaternal embryos. Nat Biotechnol, 2007. 25(9): p. 1045-50.

18. Zeira, E., et al., The knockdown of H19IncRNA reveals its regulatory role in pluripotency and tumorigenesis of human embryonic carcinoma cells. Oncotarget, 2015. 6(33): p. 34691-703.

19. Yoshimizu, T., et al., The H19 locus acts in vivo as a tumor suppressor. Proc Natl Acad Sci U S A, 2008. 105(34): p. 12417-22.

20. Takahashi, N., et al., Deleting maternal Gt/2 leads to growth enhancement and decreased expression of stem cell markers in teratoma. J Reprod Dev, 2015. 61(1): p. 712.

21. STrivers, R.L., Parent-Offspring Conflict. American Zoologist, 1974. 14(1): p. 249-264.

22. Barroca, V., et al., Paternal Insulin-like Growth Factor 2 (Igf2) Regulates Stem Cell Activity During Adulthood. EBioMedicine, 2016.

23. Begemann, M., et al., Paternally Inherited IGF2 Mutation and Growth Restriction. N Engl J Med, 2015. 373(4): p. 349-56.

24. DeChiara, T.M., A. Efstratiadis, and E.J. Robertson, A growth-deficiency phenotype in heterozygous mice carrying an insulin-like growth factor II gene disrupted by targeting. Nature, 1990. 345(6270): p. 78-80.

25. Moon, Y.S., et al., Mice lacking paternally expressed Pref-1/Dlk1 display growth retardation and accelerated adiposity. Mol Cell Biol, 2002. 22(15): p. 5585-92.

26. Leighton, P.A., et al., Disruption of imprinting caused by deletion of the $\mathrm{H} 19$ gene region in mice. Nature, 1995. 375(6526): p. 34-9.

27. Falix, F.A.T.-A.-L., M.R.S.; Gaemers, I.C.; Aronson, D.C.; Lamers, W.H., DLK1 protein expression during mouse development provides new insights into its function. ISRN Developmental Biology, 2013. 2013(Article ID 628962).

28. da Rocha, S.T., et al., Gene dosage effects of the imprinted delta-like homologue 1 (dlk1/pref1) in development: implications for the evolution of imprinting. PLoS Genet, 2009. 5(2): p. e1000392.

29. Georgiades, P., et al., Parental origin-specific developmental defects in mice with uniparental disomy for chromosome 12. Development, 2000. 127(21): p. 4719-28.

30. Andrews, P.W., et al., Embryonic stem (ES) cells and embryonal carcinoma (EC) cells: opposite sides of the same coin. Biochem Soc Trans, 2005. 33(Pt 6): p. 1526-30.

31. Enver, T., et al., Cellular differentiation hierarchies in normal and culture-adapted human embryonic stem cells. Hum Mol Genet, 2005. 14(21): p. 3129-40.

32. Yin, D., et al., DLK1: increased expression in gliomas and associated with oncogenic activities. Oncogene, 2006. 25(13): p. 1852-61.

33. Huang, J., et al., Up-regulation of DLK1 as an imprinted gene could contribute to human hepatocellular carcinoma. Carcinogenesis, 2007. 28(5): p. 1094-103.

34. Kim, Y., et al., Hypoxia-regulated delta-like 1 homologue enhances cancer cell stemness and tumorigenicity. Cancer Res, 2009. 69(24): p. 9271-80.

35. Livingstone, C., IGF2 and cancer. Endocr Relat Cancer, 2013. 20(6): p. R321-39.

36. Sievers, S., et al., IGF2/H19 imprinting analysis of human germ cell tumors (GCTs) using the methylation-sensitive single-nucleotide primer extension method reflects the origin of GCTs in different stages of primordial germ cell development. Genes Chromosomes Cancer, 2005. 44(3): p. 256-64.

37. Ross, J.A., et al., Genomic imprinting of $\mathrm{H} 19$ and insulin-like growth factor-2 in pediatric germ cell tumors. Cancer, 1999. 85(6): p. 1389-94. 
38. Kawakami, T., et al., Erasure of methylation imprint at the promoter and CTCF-binding site upstream of $\mathrm{H} 19$ in human testicular germ cell tumors of adolescents indicate their fetal germ cell origin. Oncogene, 2006. 25(23): p. 3225-36.

39. Saitou, M. and M. Yamaji, Primordial germ cells in mice. Cold Spring Harb Perspect Biol, 2012. 4(11).

40. Irie, N., W.W. Tang, and M. Azim Surani, Germ cell specification and pluripotency in mammals: a perspective from early embryogenesis. Reprod Med Biol, 2014. 13(4): p. 203-215.

41. Seki, Y., et al., Cellular dynamics associated with the genome-wide epigenetic reprogramming in migrating primordial germ cells in mice. Development, 2007. 134(14): p. 2627-38.

42. Ratajczak, M.Z., Why are hematopoietic stem cells so 'sexy'? on a search for developmental explanation. Leukemia, 2017.

43. Hart, A.H., et al., The pluripotency homeobox gene NANOG is expressed in human germ cell tumors. Cancer, 2005. 104(10): p. 2092-8.

44. Shin, D.M., et al., Molecular signature of adult bone marrow-purified very small embryonic-like stem cells supports their developmental epiblast/germ line origin. Leukemia, 2010. 24(8): p. 1450-61.

45. Kucia, M., et al., A population of very small embryonic-like (VSEL) CXCR4(+)SSEA-1(+)Oct4+ stem cells identified in adult bone marrow. Leukemia, 2006. 20(5): p. 857-69.

46. Suszynska, M., et al., Expression of the erythropoietin receptor by germline-derived cells further support for a potential developmental link between the germline and hematopoiesis. J Ovarian Res, 2014. 7: p. 66.

47. Ratajczak, J., et al., Adult murine bone marrow-derived very small embryonic-like stem cells differentiate into the hematopoietic lineage after coculture over OP9 stromal cells. Exp Hematol, 2011. 39(2): p. 225-37.

48. Lam, Y.M., et al., Histone deacetylase inhibitors induce leukemia gene expression in cord blood hematopoietic stem cells expanded ex vivo. Int J Hematol, 2017. 105(1): p. 37-43.

49. Shaikh, A., et al., Mouse Bone Marrow VSELs Exhibit Differentiation into Three Embryonic Germ Lineages and Germ \& Hematopoietic Cells in Culture. Stem Cell Rev, 2017. 13(2): p. 202-216.

50. Kerr, C.L., M.J. Shamblott, and J.D. Gearhart, Pluripotent stem cells from germ cells. Methods Enzymol, 2006. 419: p. 400-26.

51. Donovan, P.J. and M.P. de Miguel, Turning germ cells into stem cells. Curr Opin Genet Dev, 2003. 13(5): p. 463-71.

52. Biswal, B.K., et al., Acute hypersensitivity of pluripotent testicular cancer-derived embryonal carcinoma to low-dose 5-aza deoxycytidine is associated with global DNA Damage-associated p53 activation, anti-pluripotency and DNA demethylation. PLoS One, 2012. 7(12): p. e53003.

53. Wongtrakoongate, P., J. Li, and P.W. Andrews, Aza-deoxycytidine induces apoptosis or differentiation via DNMT3B and targets embryonal carcinoma cells but not their differentiated derivatives. Br J Cancer, 2014. 110(8): p. 2131-8.

54. Khan, L., et al., Testicular embryonal carcinoma presenting as chest wall subcutaneous mass. J Cytol, 2009. 26(1): p. 39-40.

55. Benton, C.B., et al., Safety and clinical activity of 5-aza-2'-deoxycytidine (decitabine) with or without Hyper-CVAD in relapsed/refractory acute lymphocytic leukaemia. Br J Haematol, 2014. 167(3): p. 356-65. 
56. Chaganti, R.S., et al., Leukemic differentiation of a mediastinal germ cell tumor. Genes Chromosomes Cancer, 1989. 1(1): p. 83-7.

57. Nichols, C.R., et al., Hematologic malignancies associated with primary mediastinal germ-cell tumors. Ann Intern Med, 1985. 102(5): p. 603-9.

58. Woodruff, K., et al., The clonal nature of mediastinal germ cell tumors and acute myelogenous leukemia. A case report and review of the literature. Cancer Genet Cytogenet, 1995. 79(1): p. 25-31.

59. Dluhosova, M., et al., Epigenetic control of SPI1 gene by CTCF and ISWI ATPase SMARCA5. PLoS One, 2014. 9(2): p. e87448.

60. Khoury, H., et al., An upstream insulator regulates DLK1 imprinting in AML. Blood, 2010. 115(11): p. 2260-3.

61. Li, L., S.J. Forman, and R. Bhatia, Expression of DLK1 in hematopoietic cells results in inhibition of differentiation and proliferation. Oncogene, 2005. 24(27): p. 4472-6.

62. Lyu, Y., et al., Dysfunction of the WT1-MEG3 signaling promotes AML leukemogenesis via p53-dependent and-independent pathways. Leukemia, 2017.

63. Yue, L.Z., et al., Expression of DLK1 Gene in the Bone Marrow Cells of Patients with Myelodysplastic Syndromes and Its Clinical Significance. Cancer Biol Med, 2012. 9(3): p. 188-91.

64. van Rhenen, A., et al., High stem cell frequency in acute myeloid leukemia at diagnosis predicts high minimal residual disease and poor survival. Clin Cancer Res, 2005. 11(18): p. 6520-7.

65. Marusyk, A., V. Almendro, and K. Polyak, Intra-tumour heterogeneity: a looking glass for cancer? Nat Rev Cancer, 2012. 12(5): p. 323-34.

66. Mohanty, A.K., A. Datta, and V. Venkatraj, A model for cancer tissue heterogeneity. IEEE Trans Biomed Eng, 2014. 61(3): p. 966-74.

67. Nomura, A., et al., CD133 initiates tumors, induces epithelial-mesenchymal transition and increases metastasis in pancreatic cancer. Oncotarget, 2015. 6(10): p. 8313-22.

68. Lou, Y.W., et al., Stage-specific embryonic antigen-4 as a potential therapeutic target in glioblastoma multiforme and other cancers. Proc Natl Acad Sci U S A, 2014. 111(7): p. 2482-7.

69. Malecki, M., et al., TRA-1-60+, SSEA-4+, POU5F1+, SOX2+, NANOG+ Clones of Pluripotent Stem Cells in the Embryonal Carcinomas of the Testes. J Stem Cell Res Ther, 2013. 3(1).

70. Grasso, C., et al., Iterative sorting reveals CD133+ and CD133-melanoma cells as phenotypically distinct populations. BMC Cancer, 2016. 16(1): p. 726.

71. Lang, F., et al., Plastic CD34 and CD38 expression in adult B-cell precursor acute lymphoblastic leukemia explains ambiguity of leukemia-initiating stem cell populations. Leukemia, 2017. 31(3): p. 731-734.

72. Quesenberry, P.J., et al., Marrow Hematopoietic Stem Cells Revisited: They Exist in a Continuum and are Not Defined by Standard Purification Approaches; Then There are the Microvesicles. Front Oncol, 2014. 4: p. 56.

73. Tesfaigzi, J. and D.M. Carlson, Cell cycle-specific expression of G(0)SPR1 in Chinese hamster ovary cells. Exp Cell Res, 1996. 228(2): p. 277-82.

74. Gunjal, P., et al., An emerging question about putative cancer stem cells in established cell lines-are they true stem cells or a fluctuating cell phenotype? J Cancer Stem Cell Res, 2015. 3.

75. Liu, G., et al., Analysis of gene expression and chemoresistance of CD133+ cancer stem cells in glioblastoma. Mol Cancer, 2006. 5: p. 67. 
76. Andrews, P.W., et al., Pluripotent embryonal carcinoma clones derived from the human teratocarcinoma cell line Tera-2. Differentiation in vivo and in vitro. Lab Invest, 1984. 50(2): p. 147-62.

77. Andrews, P.W., Retinoic acid induces neuronal differentiation of a cloned human embryonal carcinoma cell line in vitro. Dev Biol, 1984. 103(2): p. 285-93.

78. Ratajczak, M.Z., et al., CD133 Expression Strongly Correlates with the Phenotype of Very Small Embryonic-/Epiblast-Like Stem Cells. Adv Exp Med Biol, 2013. 777: p. 125-41.

79. Takahashi, M., et al., CD133 is a positive marker for a distinct class of primitive human cord blood-derived CD34-negative hematopoietic stem cells. Leukemia, 2014. 28(6): p. 1308-15.

80. Schwartz, C.M., et al., NTera2: a model system to study dopaminergic differentiation of human embryonic stem cells. Stem Cells Dev, 2005. 14(5): p. 517-34.

81. Park, E.K., et al., Transcriptional repression of cancer stem cell marker CD133 by tumor suppressor p53. Cell Death Dis, 2015. 6: p. e1964.

82. Vardiman, J.W., et al., The 2008 revision of the World Health Organization (WHO) classification of myeloid neoplasms and acute leukemia: rationale and important changes. Blood, 2009. 114(5): p. 937-51.

83. Holowiecki, J., et al., Cladribine, but not fludarabine, added to daunorubicin and cytarabine during induction prolongs survival of patients with acute myeloid leukemia: a multicenter, randomized phase III study. J Clin Oncol, 2012. 30(20): p. 2441-8.

84. Bradstock, K.F., et al., A randomized trial of high-versus conventional-dose cytarabine in consolidation chemotherapy for adult de novo acute myeloid leukemia in first remission after induction therapy containing high-dose cytarabine. Blood, 2005. 105(2): p. 481-8.

85. Cheson, B.D., et al., Revised recommendations of the International Working Group for Diagnosis, Standardization of Response Criteria, Treatment Outcomes, and Reporting Standards for Therapeutic Trials in Acute Myeloid Leukemia. J Clin Oncol, 2003. 21(24): p. 4642-9.

86. Jaglal, M.V., et al., Cladribine, cytarabine, filgrastim, and mitoxantrone (CLAG-M) compared to standard induction in acute myeloid leukemia from myelodysplastic syndrome after azanucleoside failure. Leuk Res, 2014. 38(4): p. 443-6.

87. Gomes, C., et al., RNA polymerase 1-driven transcription as a mediator of BDNF-induced neurite outgrowth. J Biol Chem, 2011. 286(6): p. 4357-63.

88. Kim, Y., Effect of retinoic acid and delta-like 1 homologue (DLK1) on differentiation in neuroblastoma. Nutr Res Pract, 2010. 4(4): p. 276-82.

89. Ohinata, Y., et al., Blimp1 is a critical determinant of the germ cell lineage in mice. Nature, 2005. 436(7048): p. 207-13.

90. Wallace, D.C. and D. Chalkia, Mitochondrial DNA genetics and the heteroplasmy conundrum in evolution and disease. Cold Spring Harb Perspect Biol, 2013. 5(11): p. a021220.

91. Medvinsky, A., S. Rybtsov, and S. Taoudi, Embryonic origin of the adult hematopoietic system: advances and questions. Development, 2011. 138(6): p. 1017-31.

92. Scaldaferri, M.L., et al., Hematopoietic activity in putative mouse primordial germ cell populations. Mech Dev, 2015. 136: p. 53-63.

93. Miwa, Y., et al., Primitive erythropoiesis of mouse teratocarcinoma stem cells $P C C 3 / A / 1$ in serum-free medium. Development, 1991. 111(2): p. 543-9.

94. Benetatos, L. and G. Vartholomatos, Imprinted genes in myeloid lineage commitment in normal and malignant hematopoiesis. Leukemia, 2015. 29(6): p. 1233-42. 
95. Lui, J.C., et al., An imprinted gene network that controls mammalian somatic growth is down-regulated during postnatal growth deceleration in multiple organs. Am J Physiol Regul Integr Comp Physiol, 2008. 295(1): p. R189-96.

96. Varrault, A., et al., Zac1 regulates an imprinted gene network critically involved in the control of embryonic growth. Dev Cell, 2006. 11(5): p. 711-22.

97. da Rocha, S.T., et al., Genomic imprinting at the mammalian DIk1-Dio3 domain. Trends Genet, 2008. 24(6): p. 306-16.

98. Manodoro, F., et al., Loss of imprinting at the $14 q 32$ domain is associated with microRNA overexpression in acute promyelocytic leukemia. Blood, 2014. 123(13): p. 2066-74.

99. Kagami, M., et al., The IG-DMR and the MEG3-DMR at human chromosome 14q32.2: hierarchical interaction and distinct functional properties as imprinting control centers. PLoS Genet, 2010. 6(6): p. e1000992.

100. Benetatos, L., et al., CPG methylation analysis of the MEG3 and SNRPN imprinted genes in acute myeloid leukemia and myelodysplastic syndromes. Leuk Res, 2010. 34(2): p. 148-53.

101. Benetatos, L., et al., Promoter hypermethylation of the MEG3 (DLK1/MEG3) imprinted gene in multiple myeloma. Clin Lymphoma Myeloma, 2008. 8(3): p. 171-5.

102. Dixon-Mclver, A., et al., Distinctive patterns of microRNA expression associated with karyotype in acute myeloid leukaemia. PLoS One, 2008. 3(5): p. e2141.

103. Jongen-Lavrencic, M., et al., MicroRNA expression profiling in relation to the genetic heterogeneity of acute myeloid leukemia. Blood, 2008. 111(10): p. 5078-85.

104. Zhang, W., et al., Effect of DLK1 on tumorigenesis in CD34+CD38- bone marrow cells in myelodysplastic syndromes. Oncol Lett, 2013. 6(1): p. 203-206.

105. Begum, A., et al., Interaction of delta-like 1 homolog (Drosophila) with prohibitins and its impact on tumor cell clonogenicity. Mol Cancer Res, 2014. 12(1): p. 155-64.

106. Benetatos, L. and E. Hatzimichael, Delta-like homologue 1 and its role in the bone marrow niche and hematologic malignancies. Clin Lymphoma Myeloma Leuk, 2014. 14(6): p. 451-5.

107. Cai, C.M., et al., Targeting endogenous DLK1 exerts antitumor effect on hepatocellular carcinoma through initiating cell differentiation. Oncotarget, 2016.

108. Fabian, K.P., et al., Therapeutic efficacy of combined vaccination against tumor pericyteassociated antigens DLK1 and DLK2 in mice. Oncoimmunology, 2017. 6(3): p. e1290035.

109. Falix, F.A., et al., Possible roles of DLK1 in the Notch pathway during development and disease. Biochim Biophys Acta, 2012. 1822(6): p. 988-95.

110. Li, L., et al., DLK1 promotes lung cancer cell invasion through upregulation of MMP9 expression depending on Notch signaling. PLoS One, 2014. 9(3): p. e91509.

111. Tierling, S., et al., High-resolution map and imprinting analysis of the Gt/2-Dnchc1 domain on mouse chromosome 12. Genomics, 2006. 87(2): p. 225-35.

112. Ma, X., et al., The effects of increased expression of DLK1 gene on the pathogenesis of myelodysplastic syndromes. Clin Lymphoma Myeloma Leuk, 2012. 12(4): p. 261-8.

113. Argiropoulos, B., et al., Meis1 disrupts the genomic imprint of Dlk1 in a NUP98-HOXD13 leukemia model. Leukemia, 2010. 24(10): p. 1788-91.

114. Takahashi, N., et al., Deletion of Gt/2, imprinted non-coding RNA, with its differentially methylated region induces lethal parent-origin-dependent defects in mice. Hum Mol Genet, 2009. 18(10): p. 1879-88.

115. Zhou, Y., X. Zhang, and A. Klibanski, MEG3 noncoding RNA: a tumor suppressor. J Mol Endocrinol, 2012. 48(3): p. R45-53. 
116. Valleron, W., et al., Specific small nucleolar RNA expression profiles in acute leukemia. Leukemia, 2012. 26(9): p. 2052-60.

117. Kagami, M., et al., Epimutations of the IG-DMR and the MEG3-DMR at the $14 q 32.2$ imprinted region in two patients with Silver-Russell Syndrome-compatible phenotype. Eur J Hum Genet, 2015. 23(8): p. 1062-7.

118. Kim, J., C.L. Bretz, and S. Lee, Epigenetic instability of imprinted genes in human cancers. Nucleic Acids Res, 2015. 43(22): p. 10689-99.

119. Diez-Torre, A., et al., The role of microenvironment in testicular germ cell tumors. Cancer Biol Ther, 2010. 10(6): p. 529-36.

120. Wu, Q., et al., Regulated expression of two sets of paternally imprinted genes is necessary for mouse parthenogenetic development to term. Reproduction, 2006. 131(3): p. 481-8.

121. Li, Z., et al., Birth of fertile bimaternal offspring following intracytoplasmic injection of parthenogenetic haploid embryonic stem cells. Cell Res, 2016. 26(1): p. 135-8.

122. van Gurp, R.J., et al., Biallelic expression of the H19 and IGF2 genes in human testicular germ cell tumors. J Natl Cancer Inst, 1994. 86(14): p. 1070-5.

123. Kim, K.P., et al., Gene-specific vulnerability to imprinting variability in human embryonic stem cell lines. Genome Res, 2007. 17(12): p. 1731-42.

124. Woodfine, K., J.E. Huddleston, and A. Murrell, Quantitative analysis of DNA methylation at all human imprinted regions reveals preservation of epigenetic stability in adult somatic tissue. Epigenetics Chromatin, 2011. 4(1): p. 1.

125. Szabo, P.E., et al., Role of CTCF binding sites in the lgf2/H19 imprinting control region. Mol Cell Biol, 2004. 24(11): p. 4791-800.

126. Yang, N.Q., et al., Crosstalk between Meg3 and miR-1297 regulates growth of testicular germ cell tumor through PTEN/PI3K/AKT pathway. Am J Transl Res, 2016. 8(2): p. 10919.

127. Bujak, E., D. Ritz, and D. Neri, A Monoclonal Antibody to Human DLK1 Reveals Differential Expression in Cancer and Absence in Healthy Tissues. Antibodies, 2015. 4(2): p. 71-87.

128. Lee, D., et al., Human soluble delta-like 1 homolog exerts antitumor effects in vitro and in vivo. Biochem Biophys Res Commun, 2016. 475(2): p. 209-15.

129. Traustadottir, G.A., et al., Evidence of non-canonical NOTCH signaling: Delta-like 1 homolog (DLK1) directly interacts with the NOTCH1 receptor in mammals. Cell Signal, 2016. 28(4): p. 246-54.

130. Traustadottir, G.A., et al., The non-canonical NOTCH1 ligand Delta-like 1 homolog (DLK1) self interacts in mammals. Int J Biol Macromol, 2017.

131. Tarnowski, M., et al., 5Azacytidine inhibits human rhabdomyosarcoma cell growth by downregulating insulinlike growth factor 2 expression and reactivating the $\mathrm{H} 19$ gene product miR675, which negatively affects insulinlike growth factors and insulin signaling. Int J Oncol, 2015. 46(5): p. 2241-50.

132. Juttermann, R., E. Li, and R. Jaenisch, Toxicity of 5-aza-2'-deoxycytidine to mammalian cells is mediated primarily by covalent trapping of DNA methyltransferase rather than DNA demethylation. Proc Natl Acad Sci U S A, 1994. 91(25): p. 11797-801.

133. Murphy, S.K., et al., Genotype-Epigenotype Interaction at the IGF2 DMR. Genes (Basel), 2015. 6(3): p. 777-89.

134. Wan, L.B. and M.S. Bartolomei, Regulation of imprinting in clusters: noncoding RNAs versus insulators. Adv Genet, 2008. 61: p. 207-23. 
135. Ly, A., et al., Alterations in tumorigenicity of embryonal carcinoma cells by IGF-I triplehelix induced changes in immunogenicity and apoptosis. Life Sci, 2000. 68(3): p. 307-19.

136. Granerus, M., et al., Insulin-like growth factor II prevents apoptosis in a human teratoma derived cell line. Clin Mol Pathol, 1995. 48(3): p. M153-7.

137. Engstrom, W., A.R. Rees, and J.K. Heath, Proliferation of a human embryonal carcinomaderived cell line in serum-free medium: inter-relationship between growth factor requirements and membrane receptor expression. J Cell Sci, 1985. 73: p. 361-73.

138. Sundgren, N.C., et al., Extracellular signal-regulated kinase and phosphoinositol-3 kinase mediate IGF-1 induced proliferation of fetal sheep cardiomyocytes. Am J Physiol Regul Integr Comp Physiol, 2003. 285(6): p. R1481-9.

139. Jensen, C.H., et al., Protein structure of fetal antigen 1 (FA1). A novel circulating human epidermal-growth-factor-like protein expressed in neuroendocrine tumors and its relation to the gene products of dlk and pG2. Eur J Biochem, 1994. 225(1): p. 83-92.

140. Puertas-Avendano, R.A., et al., Role of the non-canonical notch ligand delta-like protein 1 in hormone-producing cells of the adult male mouse pituitary. J Neuroendocrinol, 2011. 23(9): p. 849-59.

141. Zhu, N.L., et al., Hepatic stellate cell-derived delta-like homolog 1 (DLK1) protein in liver regeneration. J Biol Chem, 2012. 287(13): p. 10355-67.

142. Immervoll, H., et al., Expression of the "stem cell marker" CD133 in pancreas and pancreatic ductal adenocarcinomas. BMC Cancer, 2008. 8: p. 48.

143. Sansone, P., et al., Self-renewal of CD133(hi) cells by IL6/Notch3 signalling regulates endocrine resistance in metastatic breast cancer. Nat Commun, 2016. 7: p. 10442.

144. Watanabe, K., et al., Cripto-1 is a cell surface marker for a tumorigenic, undifferentiated subpopulation in human embryonal carcinoma cells. Stem Cells, 2010. 28(8): p. 1303-14.

145. Aloia, A., et al., The sialyl-glycolipid stage-specific embryonic antigen 4 marks a subpopulation of chemotherapy-resistant breast cancer cells with mesenchymal features. Breast Cancer Res, 2015. 17(1): p. 146.

146. Oliveros-Etter, M., et al., PGC Reversion to Pluripotency Involves Erasure of DNA Methylation from Imprinting Control Centers followed by Locus-Specific Re-methylation. Stem Cell Reports, 2015. 5(3): p. 337-49.

147. Nagamatsu, G., et al., Induction of pluripotent stem cells from primordial germ cells by single reprogramming factors. Stem Cells, 2013. 31(3): p. 479-87.

148. Nygaard, M.B., et al., Cell context-specific expression of primary cilia in the human testis and ciliary coordination of Hedgehog signalling in mouse Leydig cells. Sci Rep, 2015. 5: p. 10364.

149. Shima, Y., et al., Contribution of Leydig and Sertoli cells to testosterone production in mouse fetal testes. Mol Endocrinol, 2013. 27(1): p. 63-73.

150. Lin, H., et al., Involvement of testicular growth factors in fetal Leydig cell aggregation after exposure to phthalate in utero. Proc Natl Acad Sci U S A, 2008. 105(20): p. 7218-22.

151. Tiesman, J., et al., Production of growth factors related to fibroblast growth factor and platelet-derived growth factor by human embryonal carcinoma cells. In Vitro Cell Dev Biol, 1988. 24(12): p. 1209-16.

152. Mortensen, S.B., et al., Membrane-tethered delta-like 1 homolog (DLK1) restricts adipose tissue size by inhibiting preadipocyte proliferation. Diabetes, 2012. 61(11): p. 2814-22.

153. Ferron, S.R., et al., Postnatal loss of Dlk1 imprinting in stem cells and niche astrocytes regulates neurogenesis. Nature, 2011. 475(7356): p. 381-5. 
154. Kluth, S.M., et al., DLK-1 as a marker to distinguish unrestricted somatic stem cells and mesenchymal stromal cells in cord blood. Stem Cells Dev, 2010. 19(10): p. 1471-83.

155. Ishizawa, K., et al., Tumor-initiating cells are rare in many human tumors. Cell Stem Cell, 2010. 7(3): p. 279-82.

156. Gilbertson, R.J. and T.A. Graham, Cancer: Resolving the stem-cell debate. Nature, 2012. 488(7412): p. 462-3.

157. Jordan, C.T., Cancer stem cells: controversial or just misunderstood? Cell Stem Cell, 2009. 4(3): p. 203-5.

158. Maxmen, A., California's \$3-billion bet on stem cells faces final test. Nature, 2017. 544(7651): p. 401-402.

159. Ben-David, U. and N. Benvenisty, The tumorigenicity of human embryonic and induced pluripotent stem cells. Nat Rev Cancer, 2011. 11(4): p. 268-77.

160. Ratajczak, M.Z., et al., Very small embryonic-like stem cells (VSELs) represent a real challenge in stem cell biology: recent pros and cons in the midst of a lively debate. Leukemia, 2014. 28(3): p. 473-84.

161. Zimmerman, D.L., C.S. Boddy, and C.S. Schoenherr, Oct4/Sox2 binding sites contribute to maintaining hypomethylation of the maternal igf2/h19 imprinting control region. PLoS One, 2013. 8(12): p. e81962. 


\title{
CURRICULUM VITAE
}

\author{
Zachariah Payne Sellers
}

\author{
Work (Preferred): University of Louisville \\ Developmental Biology Program \\ Home: 1230 Troy Pike \\ Versailles, KY 40383 \\ 500 South Floyd Street \\ Louisville, KY 40204 \\ (859) 221-7711 \\ Zpsel101@louisville.edu
}

\section{$\underline{\text { Education }}$}

University of Louisville, Louisville, KY

$\mathrm{PhD}$ Program, Microbiology \& Immunology

Graduate Advisor: Dr. Mariusz Ratajczak

Current GPA: 3.36

Expected Graduation Data: Summer 2017

Eastern Kentucky University, Richmond, KY

Master of Science, Chemistry, Thesis Track

Graduate Advisor: Dr. Tanea Reed, Ph.D.

Current GPA: 3.68

Expected Graduation Date: Spring 2018

University of Kentucky, Lexington, KY

Bachelor of Science, Chemistry

Bachelor of Science, Biology

Minor, Mathematics

August 2011 - Present

August 2013 - April 2014

April 2015 - Present

August 2005 - May 2011

\section{Peer-Reviewed Publications}

Sellers, Z. P.; Bolchun, L.; Ratajczak, M. Z.; Schneider, G. Methylation at the DLKI$M E G 3$ locus predicts long-term survival of AML patients. [Manuscript in preparation].

Sellers, Z. P.; Schneider, G.; Ratajczak, M. Z. Paternal imprinting at the DLK1-MEG3 and IGF2-H19 loci identifies DLK1 as a potential therapeutic target in embryonal carcinoma [Manuscript in preparation]. 
Sellers, Z. P.; Schneider, G.; Ratajczak, M. Z. Novel evidence that pituitary sex hormones regulate migration, adhesion, and proliferation of embryonic stem cells and teratocarcinoma cells [Manuscript in review]

Sellers, Z. P.; Schneider, G.; Suszynska, M.; Bujko, K.; Pedziwiatr, D. Do cancer cell lines have fixed or fluctuating stem cell phenotypes? - studies with the NTera2 cell line. Stem Cell Reviews and Reports 2017.

Schneider, G.; Sellers, Z. P.; Bujko, K.; Kakar, S. S.; Kucia, M.; Ratajczak, M. Z. Novel pleiotropic effects of bioactive phospholipids in human lung cancer metastasis. Oncotarget 2017.

Schneider, G.; Bryndza, E.; Poniewierska-Baran, A.; Serwin, K.; Suszynska, M.; Sellers, Z. P.; Merchant, M. L.; Kaliappan, A.; Ratajczak, J.; Kucia, M.; Garbett, N. C.; Ratajczak, M. Z. Evidence that vitronectin is a potent migration-enhancing factor for cancer cells chaperoned by fibrinogen-a novel view of the metastasis of cancer cells to low-fibrinogen lymphatics and body cavities. Oncotarget 2016.

Schneider, G.; Sellers, Z. P.; Ratajczak, M. Z. Parentally imprinted genes regulate hematopoiesis - new evidence from the Dlk1-Gt12 locus. Stem Cell Investigation 2016, 3 , $1-5$.

Schneider, G.; Sellers, Z. P.; Ratajczak, M. Z. Induction of a tumor-metastasis-receptive microenvironment as an unwanted side effect after radio/chemotherapy and in vitro and in vivo assays to study this phenomenon. Methods in Molecular Biology 2016.

Schneider, G.; Glaser, T.; Lameu, C.; Abdelbaset-Ismail, A.; Sellers, Z. P.; Moniuszko, M.; Ulrich, H.; Ratajczak, M. Z. Extracellular nucleotides as novel, underappreciated prometastatic factors that stimulate purinergic signaling in human lung cancer cells. Molecular Cancer 2015, 14, 201.

Reed, T. T.; Sellers, Z. P.; Butterfield, D. A. Lipid peroxidation and age-related neurodegenerative disorders. In Lipid Oxidation in Health and Disease; Spicket, C. M and Forman, H. J. CRC Press: Boca Raton, Florida, 2015, pp 329-362.

Schneider, G.; Sellers, Z. P.; Abdel-Latif, A.; Morris, A. J.; Ratajczak, M. Z. Bioactive lipids, LPC and LPA, are novel prometastatic factors and their tissue levels increase in response to radio/chemotherapy. Molecular Cancer Research 2014, 12, 1560-1573.

Ratajczak, M. Z.; Schneider, G.; Sellers, Z. P.; Kucia, M.; Kakar, S. S. The embryonic rest hypothesis of cancer development - an old XIX century theory revised. Journal of Cancer Stem Cell Research 2014, 2, e1001.

Sellers, Z. P.; Williams, R. A.; Overbay, J. W.; Cho, J.; Henderson, M.; Reed, T. T. Current therapeutic modalities, enzyme kinetics, and redox proteomics in traumatic brain injury. In Traumatic Brain Injury; Sadaka, F.; InTECH: Rijecka, Croatia, 2014, pp 39-77. 
Johnson, J. T.; Sellers, Z. P.; Williams, R. A.; Reed, T. T. Hydroxyacids and neurodegenerative disorders. In Advances in Chemistry Research; Taylor, J. C.; Nova Science: New York, 2013, Vol. 18 pp 239-251.

Reed, T. T.; Sellers, Z. P. The use of pharmaceutical intervention as a mechanistic tool to regulate bioenergetics and inhibit free radical oxidative stress during the progression of Alzheimer's disease. Journal of Drug Metabolism and Toxicology 2012, S8:001.

Bachas-Daunert, P. G.; Sellers, Z. P.; Wei, Y. Detection of Halogenated Organic Compounds Using Immobilized Thermophilic Dehalogenase. Analytical and Bioanalytical Chemistry 2009, 395, 1173-1178. 